\title{
Supporting Information for: Thermal Conductivity from Entropy Scaling: A Group-Contribution Method
}

\author{
Madlen Hopp and Joachim Gross* \\ Institute of Thermodynamics and Thermal Process Engineering, University of Stuttgart, \\ Pfaffenwaldring 9, 70569 Stuttgart, Germany \\ E-mail: gross@itt.uni-stuttgart.de
}

Phone: +49 (0)711 685 66103. Fax: +49 (0)711 68566140 
This supporting Information details...

1. the definition of AAD.

2. the resulting parameters $A_{\alpha}-C_{\alpha}$.

3. the full legend of Fig. 1 of the manuscript.

4. diagrams supplementing Fig. 3 of the manuscript.

5. the list of components shown in Fig. 6 of the manuscript.

6. results of the full GC method for substances containing two or more functional groups.

7. the AADs for the individual approach using PCP-SAFT and the full GC method using GC PCP-SAFT, as well as the number of available data for all investigated substances.

8. the individual correlation parameters $A-D$ for PCP-SAFT for all investigated components, for which no parameters were published yet,

9. as well as the according individual PCP-SAFT parameters.

10. group decomposition of all considered substances. 


\section{Definition of AAD}

The averaged absolute deviations (AAD) is defined as

$$
A A D \%=\frac{100}{N_{\text {data }}} \sum_{i}^{N_{\text {data }}}\left(\frac{\left|\lambda_{\mathrm{i}, \text { calc }}-\lambda_{\mathrm{i}, \exp }\right|}{\lambda_{\mathrm{i}, \exp }}\right)
$$

resulting parameters $A_{\alpha}-C_{\alpha}$ 
Table S1: Group-contribution parameters $A_{\alpha}-C_{\alpha}$ for thermal conductivities based on GC PCP-SAFT.

\begin{tabular}{|c|c|c|c|}
\hline functional group $\alpha$ & $A_{\alpha}$ & $B_{\alpha}$ & $C_{\alpha}$ \\
\hline \multicolumn{4}{|c|}{$n$-alkanes } \\
\hline$-\mathrm{CH}_{3}$ & -0.022767 & -0.271017 & 0.631023 \\
\hline$-\mathrm{CH}_{2}-$ & 0.023790 & -0.000372 & -0.000737 \\
\hline $\mathrm{H}_{3} \mathrm{C}-\mathrm{CH}_{3}$ & -0.171653 & -0.395063 & 1.884401 \\
\hline $\mathrm{CH}_{4}$ & 0.083389 & -0.525218 & 1.144751 \\
\hline \multicolumn{4}{|c|}{ branched alkanes } \\
\hline$-\mathrm{CH}<$ & 0.073479 & 0.268006 & -0.715219 \\
\hline$>\mathrm{C}<$ & 0.137522 & 0.432011 & -1.603643 \\
\hline \multicolumn{4}{|c|}{ cyclic alkanes } \\
\hline$-\mathrm{CH}_{2}-\left(\mathrm{C}_{5}\right)$ & -0.023861 & -0.125883 & 0.233695 \\
\hline$-\mathrm{CH}<{ }_{\left(\mathrm{C}_{5}\right)}$ & 1.569517 & 0.030302 & -2.415547 \\
\hline$-\mathrm{CH}_{2}-\left(\mathrm{C}_{6}\right)$ & 0.021505 & -0.098933 & 0.171388 \\
\hline$-\mathrm{CH}<{ }_{\left(\mathrm{C}_{6}\right)}$ & $\begin{array}{r}0.081725 \\
\text { alkenes }\end{array}$ & -0.001426 & -0.962725 \\
\hline $\mathrm{H}_{2} \mathrm{C}=$ & 0.118742 & -0.212624 & 0.603502 \\
\hline$-\mathrm{HC}=$ & -0.149712 & -0.100910 & -0.053894 \\
\hline$>\mathrm{C}=$ & $\begin{array}{c}-0.040429 \\
\text { aldehydes }\end{array}$ & 0.281085 & -0.591450 \\
\hline$-\mathrm{CH}=\mathrm{O}$ & $\begin{array}{l}0.108913 \\
\text { aromatics }\end{array}$ & -0.536623 & -0.229006 \\
\hline$-\mathrm{CH}-$ (aromatic) & 0.020046 & -0.083404 & 0.208123 \\
\hline$>\mathrm{C}-($ aromatic $)$ & $\begin{array}{r}0.152283 \\
\text { esters }\end{array}$ & 0.115018 & -0.756362 \\
\hline $\mathrm{HCOO}-$ & 0.139924 & -0.390715 & 0.141069 \\
\hline$-\mathrm{COO}-$ & $\begin{array}{r}-0.047422 \\
\text { ethers* }\end{array}$ & -0.094117 & -0.217276 \\
\hline$-\mathrm{O}-\mathrm{CH}_{3}$ & 0.013269 & 0.037753 & 0.077359 \\
\hline$-\mathrm{O}-\mathrm{CH}_{2}-$ & -0.279392 & -0.455717 & 0.458677 \\
\hline$-\mathrm{O}-$ & $\begin{array}{r}-0.900976 \\
\text { ketones }\end{array}$ & 0.253726 & 1.716688 \\
\hline$>\mathrm{C}=\mathrm{O}$ & $\begin{array}{l}-0.209035 \\
\text { alcohols }^{* *}\end{array}$ & 0.052247 & 0.226533 \\
\hline$-\mathrm{OH}$ & 0.095944 & 0.227526 & 1.182451 \\
\hline $\mathrm{CH}_{3} \mathrm{OH}$ & $\begin{array}{r}-0.558730 \\
\text { others }\end{array}$ & -0.434812 & 0.894255 \\
\hline$-\mathrm{NH}_{2}$ & 0.260570 & -0.062493 & 0.661768 \\
\hline$>\mathrm{NH}$ & 0.185228 & 0.238233 & 0.393120 \\
\hline $\mathrm{CO}_{2}$ & -0.577272 & -0.515448 & 1.671248 \\
\hline $\mathrm{H}_{2} \mathrm{O}$ & -0.392458 & -0.120720 & 2.717849 \\
\hline $\mathrm{HC} \equiv \mathrm{CH}$ & -0.088542 & -0.666847 & -0.673819 \\
\hline
\end{tabular}

*: for ethers, use $-\mathrm{O}-\mathrm{CH}_{3}$ group, if possible.

Otherwise use $-\mathrm{O}-\mathrm{CH}_{2}-$ group, if possible.

Otherwise use - $\mathrm{O}-$ group (for branched ethers).

**: $D_{\mathrm{OH}}=D_{\mathrm{CH}_{3} \mathrm{OH}}=0.0038$ 


\section{Full Legend of Fig. 1}

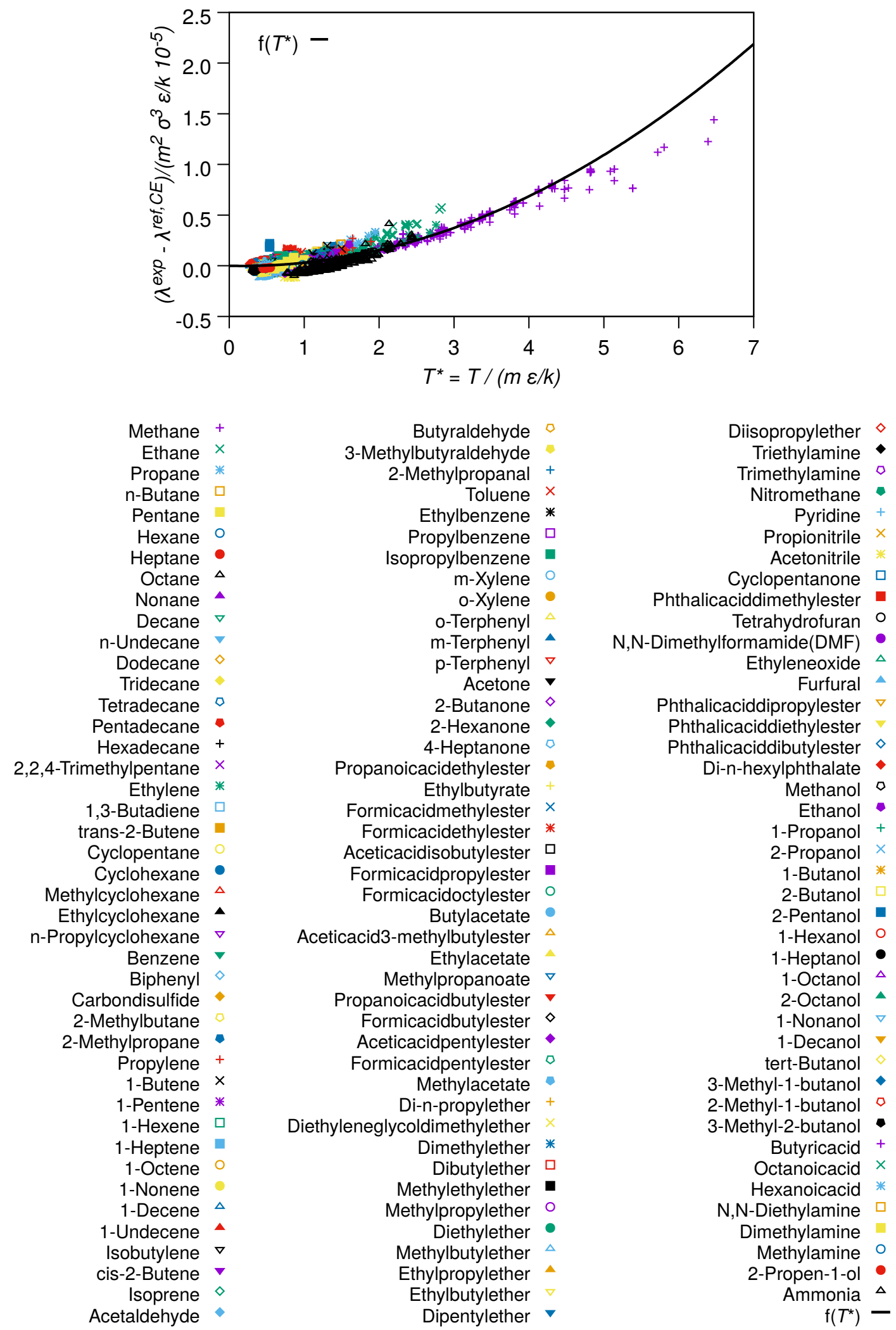




\section{Additions to Fig. 3}

As an addition to Fig. 3, we give here the diagrams for a rather small molecule, Methane, as well. Furthermore, deviations over the range of reduced residual entropy are given for the substances of Fig. 3 and Methane. The high deviations in the gas phase reult from the definition of deviation.
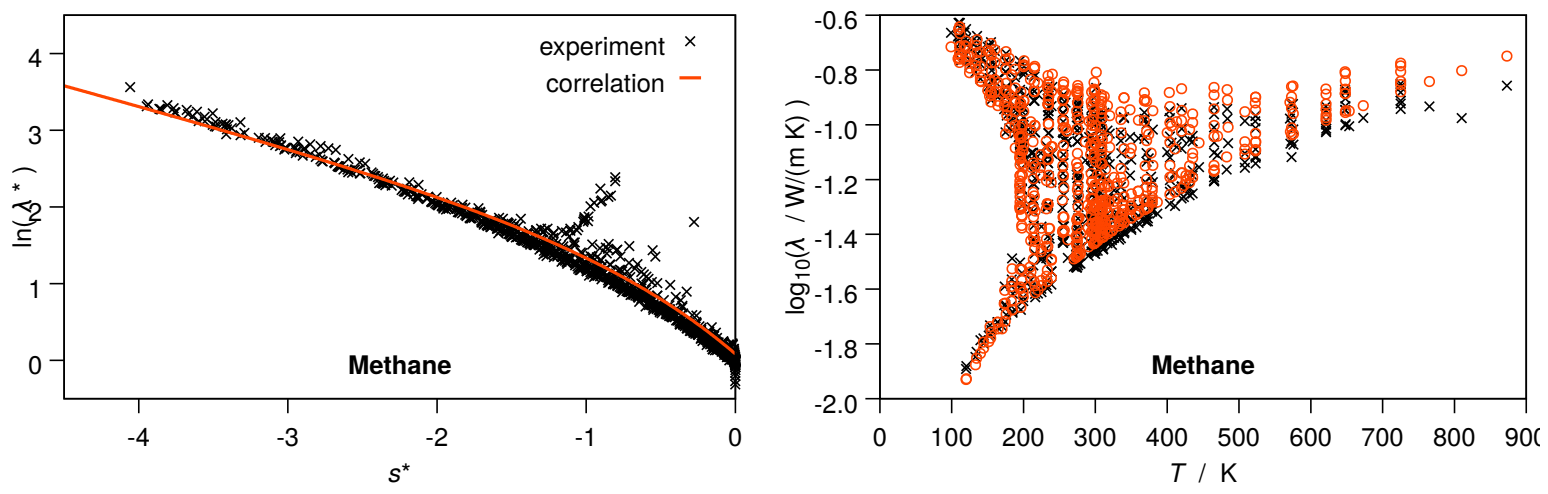

Figure S1: Thermal conductivity of methane. Left diagrams show the logarithmic reduced thermal conductivity $\ln \left(\lambda_{*}\right)$ versus reduced residual entropy $s^{*}$. Right diagrams give thermal conductivities for varying temperature. Comparison of the proposed group-contribution entropy scaling model (red line and red circles) to experimental data (black crosses). 

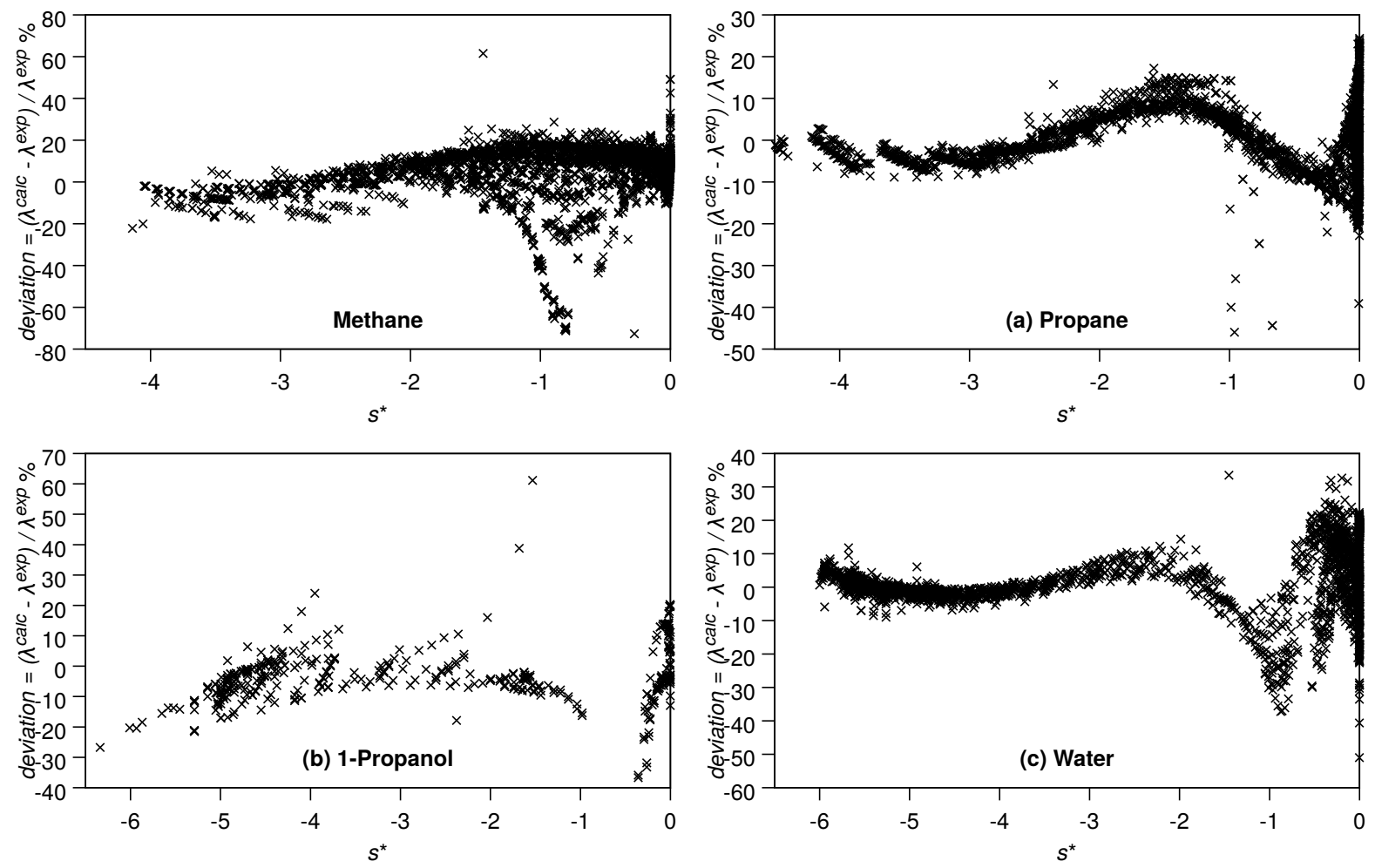

Figure S2: Deviations of calculated to experimental thermal conductivity of methane, npropane, 1-propanol and water. 
Components of Fig. 6

Table S2:

\begin{tabular}{llccll} 
\# & group & $\begin{array}{c}\text { number of } \\
\text { functional groups }\end{array}$ & $\begin{array}{c}\text { number of } \\
\text { points }\end{array}$ & & \\
\hline 1 & Cyclo & 4 & 2 & 6.091 & Bicyclopentyl \\
2 & Cyclo & 4 & 2 & 23.690 & Bicyclohexyl \\
3 & Cyclo & 6 & 2 & 25.892 & Dicyclohexylmethane \\
4 & Cyclo & 5 & 2 & 17.854 & $1,1-$ Dicyclohexylheptane \\
5 & Aromatic & 37 & 2 & 27.326 & Biphenyl \\
6 & Aromatic & 26 & 2 & 12.489 & Diphenylmethane \\
7 & Aromatic & 53 & 2 & 10.508 & Ditolylmethane \\
8 & Aromatic & 24 & 2 & 11.925 & Isopropylbiphenyl \\
9 & Aromatic & 37 & 2 & 11.981 & Bis(isopropylphenyl)methane \\
10 & Aromatic & 28 & 3 & 11.753 & o-Terphenyl \\
11 & Aromatic & 22 & 3 & 22.234 & m-Terphenyl \\
12 & Aromatic & 12 & 3 & 28.730 & p-Terphenyl \\
13 & Ester & 256 & 2 & 27.520 & Diethylsuccinate \\
14 & Ester & 171 & 30.384 & Dipropylsuccinate \\
\hline
\end{tabular}

Continued on next page 
Table - continued from previous page

\begin{tabular}{|c|c|c|c|c|c|}
\hline$\#$ & group & $\begin{array}{l}\text { number of } \\
\text { functional groups }\end{array}$ & $\begin{array}{c}\text { number of } \\
\text { points }\end{array}$ & $\mathrm{AAD} / \%$ & name \\
\hline 15 & Ester & 73 & 2 & 21.035 & Decanedioicaciddibutylester \\
\hline 16 & Ester & 159 & 2 & 15.690 & Di-n-hexyladipate \\
\hline 17 & Ester & 201 & 2 & 24.320 & Diisobutylsebacate \\
\hline 18 & Ester & 173 & 2 & 14.467 & Di-n-heptyladipate \\
\hline 19 & Ester & 4 & 2 & 4.920 & Di(2-ethylhexyl)adipate \\
\hline 20 & Ester & 186 & 2 & 13.839 & Hexanedioicaciddioctylester \\
\hline 21 & Ester & 180 & 2 & 15.150 & Succinicaciddinonylester \\
\hline 22 & Ether & 185 & 2 & 30.990 & Dimethoxymethane \\
\hline 23 & Ether & 165 & 2 & 30.870 & 1,2-Dimethoxyethane \\
\hline 24 & Ether & 7 & 2 & 23.761 & 1,2-Diethoxyethane \\
\hline 25 & Ether & 10 & 2 & 8.405 & Dibutoxymethane \\
\hline 26 & Ether & 6 & 2 & 11.375 & Ethyleneglycoldi-n-butylether \\
\hline 27 & Ether & 14 & 3 & 13.964 & Diethyleneglycoldimethylether \\
\hline 28 & Ether & 43 & 3 & 15.787 & Diethyleneglycoldiethylether \\
\hline 29 & Ether & 42 & 3 & 6.156 & Diethyleneglycoldibutylether \\
\hline 30 & Ether & 5 & 4 & 28.516 & Triethyleneglycoldimethylether \\
\hline
\end{tabular}




\section{Table - continued from previous page}

\begin{tabular}{cccccc}
$\#$ & group & $\begin{array}{c}\text { number of } \\
\text { functional groups }\end{array}$ & $\begin{array}{c}\text { number of } \\
\text { points }\end{array}$ \\
\hline 31 & Ether & 4 & 5 & 26.780 & Tetraethyleneglycoldimethylether \\
\hline \hline
\end{tabular}




\section{Substances with different functional groups}

Results for substances that contain two different functional groups are presented in Table S3. The table reports averaged absolute deviations of predicted thermal conductivities from experimental values. Each cell in the table represents a sub-group of substances that all have a certain combination of functional groups. The table states averaged absolute deviations for a sub-group (as the bottom-value of the cell), the number of considered substances (top left), and the number of available data points (top right). For empty cells, no experimental data of thermal conductivity where found for substances falling into this sub-group. For most of the considered substances the model gives surprisingly good predictions, considering that substances with multiple functional groups are a particularly demanding test case for groupcontribution approaches. Considering substances with different functional groups, where Table S3: Average absolute deviations for substances containing two different functional groups (AAD in \%, as bottom values of cells), the corresponding number of substances (top left) and number of experimental data points (top right).

\begin{tabular}{|c|c|c|c|c|c|c|}
\hline functional groups & Alcohol & Aldehyde & Alkene & Aromatic & Ester & Cyclic \\
\hline $\begin{array}{l}\text { Contains } \\
\text { Nitrogen }\end{array}$ & $\begin{array}{c}1 \\
68.79\end{array}$ & $\begin{array}{l}17.83 \\
\end{array}$ & & $\begin{array}{lr}1 & 24 \\
20.79\end{array}$ & & $\begin{array}{lr}2 & 7 \\
16.70\end{array}$ \\
\hline Ketone & & & & $\begin{array}{lr}2 & 23 \\
49.10\end{array}$ & $\begin{array}{l}1 \quad 6 \\
27.01\end{array}$ & $\begin{array}{lr}2 & 25 \\
24.83\end{array}$ \\
\hline Ether & $\begin{array}{c}6 \quad 160 \\
9.53\end{array}$ & & & & $\begin{array}{c}3 \quad 286 \\
62.88\end{array}$ & \\
\hline Ester & & & $\begin{array}{lr}5 & 549 \\
22.25\end{array}$ & $\begin{array}{lr}4 & 621 \\
16.81\end{array}$ & & \\
\hline Cyclic & $\begin{array}{c}2 r \\
38.31\end{array}$ & & $\begin{array}{cr}3 & 95 \\
62.89\end{array}$ & & & \\
\hline Aromatic & $\begin{array}{lr}3 & 37 \\
6.11\end{array}$ & $\begin{array}{lr}1 & 9 \\
13.82 & \end{array}$ & $\begin{array}{lr}1 & 14 \\
26.92\end{array}$ & & & \\
\hline Alkene & $\begin{array}{l}2 r \\
16.86\end{array}$ & $179.699^{5}$ & & & & \\
\hline
\end{tabular}

any functional group can occur more than once (not explicitly shown here), we saw that combinations from esters, alkenes and aromatics (in addition to normal and branched alkane functional groups) lead to good predictive results (18 substances, 1412 pts, AAD 15.65\%). 


\section{Averaged Absolute Deviations}

Averaged absolute deviations of all considered substances, for the individual approach with PCP-SAFT and the full GC approach with GC PCP-SAFT. Components are sorted by chemical families. Here, an additional identifier "SI-Symbol" is introduced, which is used in the table stating the group decomposition at the end of the Supporting Information. $s_{\text {crit }}^{*}$ calculated from GC PCP-SAFT, resulting in arithmetic mean $\bar{s}_{\text {crit }}^{*}=-0,491$.

Table S4:

\begin{tabular}{|c|c|c|c|c|c|}
\hline name & SI-Symbol & $\begin{array}{r}\text { number of } \\
\text { points }\end{array}$ & $\begin{array}{l}s_{\text {crit }}^{*} \\
-\end{array}$ & $\begin{array}{r}\text { AAD / \% } \\
\text { full GC method }\end{array}$ & $\begin{array}{r}\text { AAD / \% } \\
\text { individual method }\end{array}$ \\
\hline \multicolumn{6}{|c|}{ Alkanes } \\
\hline Methane & A-1 & 3215 & -0.070 & 10.899 & 8.025 \\
\hline Ethane & A-2 & 1486 & -0.079 & 14.874 & 7.803 \\
\hline Propane & A-3 & 2888 & -0.080 & 6.168 & 7.598 \\
\hline Butane & A-4 & 2931 & -0.089 & 4.936 & 5.148 \\
\hline Pentane & A-5 & 487 & -0.098 & 4.678 & 2.571 \\
\hline Hexane & A-6 & 650 & -0.106 & 4.000 & 3.243 \\
\hline Heptane & $A-7$ & 1332 & -0.113 & 4.959 & 3.786 \\
\hline Octane & A-8 & 495 & -0.121 & 5.992 & 3.022 \\
\hline Nonane & A-9 & 533 & -0.128 & 4.755 & 3.209 \\
\hline Decane & $\mathrm{A}-10$ & 755 & -0.134 & 4.097 & 2.891 \\
\hline Undecane & A-11 & 453 & -0.141 & 4.336 & 2.876 \\
\hline Dodecane & A-12 & 365 & -0.148 & 4.533 & 4.061 \\
\hline Tridecane & $\mathrm{A}-13$ & 613 & -0.154 & 3.120 & 2.795 \\
\hline Tetradecane & $\mathrm{A}-14$ & 474 & -0.160 & 3.336 & 2.451 \\
\hline Pentadecane & $\mathrm{A}-15$ & 368 & -0.166 & 3.076 & 1.965 \\
\hline Hexadecane & A- 16 & 466 & -0.172 & 3.122 & 2.117 \\
\hline Heptadecane & $\mathrm{A}-17$ & 352 & -0.178 & 3.333 & 4.117 \\
\hline Octadecane & $\mathrm{A}-18$ & 369 & -0.183 & 3.436 & 3.736 \\
\hline Nonadecane & A-19 & 327 & -0.189 & 3.873 & 2.466 \\
\hline Eicosane & A-20 & 55 & -0.194 & 4.994 & 0.862 \\
\hline
\end{tabular}


Table - continued from previous page

\begin{tabular}{|c|c|c|c|c|c|}
\hline name & SI-Symbol & $\begin{array}{r}\text { number of } \\
\text { points }\end{array}$ & $s_{\text {crit }}^{*}$ & $\begin{array}{r}\mathrm{AAD} / \% \\
\text { full GC method }\end{array}$ & $\begin{array}{r}\text { AAD / \% } \\
\text { individual method }\end{array}$ \\
\hline Heneicosane & $\mathrm{A}-21$ & 48 & -0.199 & 5.744 & 0.390 \\
\hline Docosane & A-22 & 54 & -0.205 & 5.719 & 1.276 \\
\hline Tricosane & $\mathrm{A}-23$ & 42 & -0.210 & 6.206 & 0.321 \\
\hline Tetracosane & $\mathrm{A}-24$ & 55 & -0.215 & 6.041 & 1.769 \\
\hline \multicolumn{6}{|c|}{ Branched Alkanes } \\
\hline 2-Methylpropane & B-1 & 1714 & -0.255 & 6.421 & 5.707 \\
\hline 2-Methylbutane & B-2 & 67 & -0.280 & 3.271 & 1.993 \\
\hline 2-Methylpentane & B-3 & 17 & -0.305 & 1.881 & 0.776 \\
\hline 2,2-Dimethylbutane & B-4 & 17 & -0.269 & 1.728 & 0.771 \\
\hline 2,3-Dimethylbutane & B-5 & 81 & -0.296 & 4.444 & 1.947 \\
\hline 3-Methylpentane & B-6 & 20 & -0.305 & 2.377 & 1.629 \\
\hline 2,4-Dimethylpentane & B-7 & 17 & -0.320 & 2.535 & 0.486 \\
\hline 2,2,3-Trimethylbutane & B-8 & 16 & -0.286 & 6.477 & 0.630 \\
\hline 3,3-Dimethylpentane & B-9 & 16 & -0.294 & 2.269 & 0.315 \\
\hline 2,3-Dimethylpentane & B-10 & 27 & -0.320 & 5.675 & 0.423 \\
\hline 3-Methylhexane & B-11 & 26 & -0.327 & 1.821 & 0.255 \\
\hline 2,2-Dimethylpentane & B-12 & 15 & -0.294 & 0.414 & 0.285 \\
\hline 2-Methylhexane & B-13 & 16 & -0.327 & 1.764 & 0.308 \\
\hline 3-Ethylpentane & B-14 & 16 & -0.327 & 3.522 & 0.227 \\
\hline 2,2,4-Trimethylpentane & B-15 & 338 & -0.310 & 3.889 & 2.421 \\
\hline 2,3,4-Trimethyl pentane & B-16 & 15 & -0.334 & 8.790 & 0.216 \\
\hline 3-Methylheptane & B-17 & 16 & -0.349 & 2.467 & 0.385 \\
\hline 2-Methylheptane & B-18 & 16 & -0.349 & 1.599 & 0.256 \\
\hline \multicolumn{6}{|c|}{ Alkene } \\
\hline Ethylene & $\mathrm{C}-1$ & 493 & -0.108 & 8.578 & 6.099 \\
\hline Propylene & $\mathrm{C}-2$ & 351 & -0.117 & 7.888 & 6.035 \\
\hline 1-Butene & $\mathrm{C}-3$ & 31 & -0.130 & 8.251 & 9.085 \\
\hline Isobutylene & $\mathrm{C}-4$ & 65 & -0.143 & 18.612 & 18.677 \\
\hline cis-2-Butene & $\mathrm{C}-5$ & 20 & -0.140 & 10.802 & 6.574 \\
\hline
\end{tabular}


Table - continued from previous page

\begin{tabular}{|c|c|c|c|c|c|}
\hline \multirow[t]{2}{*}{ name } & \multirow[t]{2}{*}{ SI-Symbol } & \multirow{2}{*}{$\begin{array}{r}\text { number of } \\
\text { points }\end{array}$} & \multirow[t]{2}{*}{$s_{\mathrm{crit}}^{*}$} & \multirow{2}{*}{$\begin{array}{r}\text { AAD / \% } \\
\text { full GC method }\end{array}$} & \multirow{2}{*}{$\begin{array}{r}\text { AAD / \% } \\
\text { individual method }\end{array}$} \\
\hline & & & & & \\
\hline trans-2-Butene & C-6 & 23 & -0.140 & 11.357 & 5.838 \\
\hline 2-Methylbuta-1,3-dien & $\mathrm{C}-7$ & 38 & -0.152 & 16.704 & 3.284 \\
\hline 1-Pentene & $\mathrm{C}-8$ & 32 & -0.143 & 7.972 & 1.983 \\
\hline 2,3-Dimethyl-1-butene & C-9 & 18 & -0.163 & 2.589 & 0.143 \\
\hline 2,3-Dimethyl-2-butene & $\mathrm{C}-10$ & 18 & -0.187 & 6.265 & 0.265 \\
\hline 1-Hexene & $\mathrm{C}-11$ & 312 & -0.156 & 3.932 & 3.464 \\
\hline 1-Heptene & $\mathrm{C}-12$ & 415 & -0.167 & 4.212 & 2.986 \\
\hline 2-Heptene & $\mathrm{C}-13$ & 34 & -0.176 & 7.395 & 1.178 \\
\hline 1-Octene & $\mathrm{C}-14$ & 430 & -0.178 & 5.226 & 4.230 \\
\hline 1-Nonene & $\mathrm{C}-15$ & 335 & -0.189 & 6.727 & 3.454 \\
\hline 1-Decene & $\mathrm{C}-16$ & 304 & -0.199 & 5.949 & 2.264 \\
\hline 1-Undecene & $\mathrm{C}-17$ & 257 & -0.210 & 3.671 & 2.255 \\
\hline 1-Dodecene & $\mathrm{C}-18$ & 290 & -0.219 & 3.101 & 2.327 \\
\hline 1-Tridecene & C-19 & 246 & -0.229 & 1.892 & 2.290 \\
\hline 1-Tetradecene & $\mathrm{C}-20$ & 271 & -0.238 & 1.962 & 2.313 \\
\hline 1-Pentadecene & $\mathrm{C}-21$ & 249 & -0.247 & 1.855 & 2.468 \\
\hline 1-Hexadecene & C-22 & 249 & -0.256 & 2.277 & 2.600 \\
\hline \multicolumn{6}{|c|}{ Cyclic Alkanes } \\
\hline Cyclopentane & D-1 & 142 & -0.297 & 1.542 & 1.508 \\
\hline Cyclohexane & D-2 & 642 & -0.298 & 6.312 & 4.932 \\
\hline Methylcyclohexane & D-3 & 1398 & -0.313 & 6.153 & 6.967 \\
\hline Ethylcyclohexane & $\mathrm{D}-4$ & 333 & -0.338 & 5.855 & 3.498 \\
\hline cis-1,2-Dimethylcyclohexane & D-5 & 21 & -0.327 & 12.365 & 0.334 \\
\hline trans-1,4-Dimethylcyclohexane & D-6 & 50 & -0.327 & 8.197 & 0.722 \\
\hline cis-1,3-Dimethyl cyclohexane & $\mathrm{D}-7$ & 44 & -0.327 & 7.851 & 0.663 \\
\hline trans-1,2-Dimethylcyclohexane & $\mathrm{D}-8$ & 36 & -0.327 & 7.933 & 1.032 \\
\hline n-Propyl cyclohexane & D-9 & 668 & -0.363 & 3.809 & 1.837 \\
\hline Methylcyclopentane & D-10 & 16 & -0.307 & 2.358 & - \\
\hline
\end{tabular}


Table - continued from previous page

\begin{tabular}{|c|c|c|c|c|c|}
\hline name & SI-Symbol & $\begin{array}{r}\text { number of } \\
\text { points }\end{array}$ & $s_{\text {crit }}^{*}$ & $\begin{array}{r}\text { AAD / \% } \\
\text { full GC method }\end{array}$ & $\begin{array}{r}\text { AAD / \% } \\
\text { individual method }\end{array}$ \\
\hline 1,2-Dimethylcyclohexane & D-11 & 4 & -0.327 & 16.727 & - \\
\hline \multicolumn{6}{|c|}{ Aldehydes } \\
\hline Butyraldehyde & E-1 & 80 & -0.180 & 6.650 & 3.889 \\
\hline 2-Methylpropanal & E-2 & 67 & -0.175 & 5.249 & 5.045 \\
\hline Pentanal & E-3 & 8 & -0.191 & 5.096 & 0.192 \\
\hline 3-Methylbutyraldehyde & E-4 & 54 & -0.187 & 3.389 & 2.292 \\
\hline Heptanal & E-5 & 12 & -0.214 & 3.659 & 0.421 \\
\hline Decanal & E-6 & 21 & -0.247 & 6.499 & 1.250 \\
\hline Dodecanal & E-7 & 16 & -0.268 & 13.704 & 1.515 \\
\hline \multicolumn{6}{|c|}{ Aromatics } \\
\hline Benzene & $\mathrm{F}-1$ & 947 & -0.187 & 7.692 & 3.152 \\
\hline Toluene & $\mathrm{F}-2$ & 3435 & -0.198 & 3.923 & 8.927 \\
\hline Ethylbenzene & F-3 & 560 & -0.213 & 6.195 & 2.686 \\
\hline p-Xylene & F-4 & 506 & -0.210 & 2.513 & 1.962 \\
\hline m-Xylene & F-5 & 485 & -0.210 & 5.558 & 3.144 \\
\hline o-Xylene & F-6 & 552 & -0.210 & 4.140 & 1.963 \\
\hline Propylbenzene & $\mathrm{F}-7$ & 94 & -0.228 & 3.137 & 1.973 \\
\hline 1,3,5-Trimethylbenzene & F-8 & 82 & -0.221 & 8.172 & 1.615 \\
\hline 1,2,3-Trimethylbenzene & F-9 & 18 & -0.221 & 3.399 & 0.259 \\
\hline 1,2,4-Trimethylbenzene & $\mathrm{F}-10$ & 117 & -0.221 & 4.586 & 1.478 \\
\hline Isopropylbenzene & $\mathrm{F}-11$ & 358 & -0.223 & 2.961 & 16.422 \\
\hline Butylbenzene & F-12 & 98 & -0.241 & 8.228 & 2.011 \\
\hline 1,2-Diethylbenzene & $\mathrm{F}-13$ & 11 & -0.238 & 1.422 & 0.751 \\
\hline sec-Butylbenzene & $\mathrm{F}-14$ & 11 & -0.237 & 2.067 & 0.544 \\
\hline tert-Butylbenzene & F-15 & 104 & -0.216 & 5.019 & 1.614 \\
\hline 1,3,5-Triisopropylbenzene & F-16 & 4 & -0.286 & 19.743 & 0.105 \\
\hline \multicolumn{6}{|c|}{ Ketones } \\
\hline Acetone & G-1 & 373 & -0.171 & 11.664 & 6.806 \\
\hline 2-Butanone & G-2 & 334 & -0.179 & 5.859 & 4.718 \\
\hline
\end{tabular}


Table - continued from previous page

\begin{tabular}{|c|c|c|c|c|c|}
\hline \multirow[t]{2}{*}{ name } & \multirow[t]{2}{*}{ SI-Symbol } & \multirow{2}{*}{$\begin{array}{r}\text { number of } \\
\text { points }\end{array}$} & \multirow[t]{2}{*}{$s_{\text {crit }}^{*}$} & \multirow{2}{*}{$\begin{array}{r}\text { AAD / \% } \\
\text { full GC method }\end{array}$} & \multirow{2}{*}{$\begin{array}{r}\text { AAD / \% } \\
\text { individual method }\end{array}$} \\
\hline & & & & & \\
\hline 2-Pentanone & G-3 & 174 & -0.189 & 3.522 & 2.415 \\
\hline 3-Pentanone & G-4 & 22 & -0.189 & 4.184 & 1.515 \\
\hline 4-Methyl-2-pentanone & G-5 & 9 & -0.196 & 0.810 & 0.119 \\
\hline 3-Hexanone & G-6 & 163 & -0.200 & 2.566 & 1.994 \\
\hline 2-Hexanone & G-7 & 103 & -0.200 & 10.769 & 6.090 \\
\hline 3-Heptanone & G-8 & 175 & -0.212 & 4.876 & 1.765 \\
\hline 2-Heptanone & G-9 & 11 & -0.212 & 4.996 & 0.488 \\
\hline 4-Heptanone & G-10 & 86 & -0.212 & 7.407 & 7.505 \\
\hline 2-Octanone & G-11 & 30 & -0.223 & 3.996 & 1.012 \\
\hline 5 -Nonanone & G-12 & 168 & -0.234 & 2.409 & 1.326 \\
\hline 6-Undecanone & G-13 & 184 & -0.255 & 3.223 & 1.084 \\
\hline 2-Decanone & G-14 & 170 & -0.245 & 2.976 & - \\
\hline 2-Undecanone & G-15 & 171 & -0.255 & 2.975 & - \\
\hline 3-Dodecanone & G-16 & 172 & -0.266 & 3.655 & - \\
\hline 7-Tridecanone & G-17 & 11 & -0.276 & 7.380 & - \\
\hline 6,10-Dimethyl-2-undecanone & G-18 & 6 & -0.269 & 8.574 & - \\
\hline \multicolumn{6}{|c|}{ Ester } \\
\hline Formic acid methyl ester & $\mathrm{H}-1$ & 11 & -0.099 & 16.536 & 0.613 \\
\hline Formic acid ethyl ester & $\mathrm{H}-2$ & 148 & -0.103 & 12.635 & 8.853 \\
\hline Formic acid propyl ester & $\mathrm{H}-3$ & 201 & -0.110 & 12.095 & 5.433 \\
\hline Formic acid butyl ester & $\mathrm{H}-4$ & 135 & -0.116 & 5.622 & 3.018 \\
\hline Formic acid pentyl ester & H-5 & 194 & -0.123 & 6.559 & 4.289 \\
\hline Formic acid heptyl ester & H-6 & 10 & -0.136 & 3.610 & 2.930 \\
\hline Formic acid octyl ester & $\mathrm{H}-7$ & 57 & -0.143 & 8.864 & 10.058 \\
\hline Acetic acid methyl ester & H-8 & 222 & -0.104 & 8.920 & 7.409 \\
\hline Acetic acid ethyl ester & H-9 & 253 & -0.109 & 8.176 & 8.063 \\
\hline Acetic acid 1-methyl-ethyl ester & $\mathrm{H}-10$ & 21 & -0.113 & 3.874 & 1.232 \\
\hline Acetic acid butyl ester & $\mathrm{H}-11$ & 198 & -0.122 & 8.254 & 7.914 \\
\hline Acetic acid 2-methyl-propyl ester & $\mathrm{H}-12$ & 125 & -0.120 & 7.410 & 4.740 \\
\hline
\end{tabular}


Table - continued from previous page

\begin{tabular}{|c|c|c|c|c|c|}
\hline name & SI-Symbol & & $s_{\text {crit }}^{*}$ & $\mathrm{AAD} / \%$ & $\mathrm{AAD} / \%$ \\
\hline & & points & & full GC method & individual method \\
\hline Acetic acid pentyl ester & $\mathrm{H}-13$ & 154 & -0.129 & 15.877 & 10.337 \\
\hline Acetic acid 3-methyl-butyl ester & $\mathrm{H}-14$ & 95 & -0.126 & 16.462 & 9.629 \\
\hline Acetic acid hexyl ester & $\mathrm{H}-15$ & 202 & -0.135 & 5.716 & 3.171 \\
\hline Acetic acid heptyl ester & $\mathrm{H}-16$ & 207 & -0.141 & 3.531 & 3.130 \\
\hline Acetic acid 2-ethyl-hexyl ester & $\mathrm{H}-17$ & 125 & -0.146 & 4.091 & 0.907 \\
\hline Butanoic acid ethyl ester & $\mathrm{H}-18$ & 218 & -0.122 & 4.386 & 3.648 \\
\hline Propanoic acid methyl ester & $\mathrm{H}-19$ & 99 & -0.109 & 7.195 & 6.955 \\
\hline Propanoic acid butyl ester & $\mathrm{H}-20$ & 131 & -0.129 & 7.402 & 2.920 \\
\hline Octadecanoic acid butyl ester & $\mathrm{H}-21$ & 13 & -0.214 & 6.724 & 0.267 \\
\hline Formic acid 1-methyl-ethyl ester & $\mathrm{H}-22$ & 87 & -0.107 & 6.968 & - \\
\hline Formic acid 3-methyl-butyl ester & $\mathrm{H}-23$ & 97 & -0.121 & 7.635 & - \\
\hline Formic acid hexyl ester & $\mathrm{H}-24$ & 213 & -0.130 & 4.133 & - \\
\hline Formic acid 4-methyl-pentyl ester & $\mathrm{H}-25$ & 144 & -0.127 & 13.520 & - \\
\hline Acetic acid octadecyl ester & $\mathrm{H}-26$ & 5 & -0.204 & 12.956 & - \\
\hline Butanoic acid 1-methyl-ethyl ester & $\mathrm{H}-27$ & 16 & -0.126 & 4.837 & - \\
\hline Butanoic acid pentyl ester & $\mathrm{H}-28$ & 10 & -0.141 & 5.129 & - \\
\hline Butanoic acid 3-methyl-butyl ester & $\mathrm{H}-29$ & 98 & -0.139 & 6.729 & - \\
\hline Butanoic acid heptyl ester & $\mathrm{H}-30$ & 159 & -0.154 & 5.492 & - \\
\hline Propanoic acid pentyl ester & $\mathrm{H}-31$ & 120 & -0.135 & 4.835 & - \\
\hline Propanoic acid 3-methyl-butyl ester & H-32 & 126 & -0.133 & 8.293 & - \\
\hline Propanoic acid hexyl ester & H-33 & 12 & -0.141 & 5.441 & - \\
\hline Propanoic acid heptyl ester & H-34 & 93 & -0.148 & 7.069 & - \\
\hline Propanoic acid octyl ester & H-35 & 98 & -0.154 & 5.221 & - \\
\hline Pentanoic acid methyl ester & H-36 & 16 & -0.122 & 5.480 & - \\
\hline Pentanoic acid pentyl ester & H-37 & 154 & -0.148 & 8.023 & - \\
\hline Pentanoic acid 3-methyl-butyl ester & H-38 & 141 & -0.146 & 5.852 & - \\
\hline Hexanoic acid methyl ester & H-39 & 292 & -0.129 & 4.982 & - \\
\hline Hexanoic acid ethyl ester & $\mathrm{H}-40$ & 160 & -0.135 & 3.740 & - \\
\hline Hexanoic acid propyl ester & $\mathrm{H}-41$ & 109 & -0.141 & 2.886 & - \\
\hline
\end{tabular}


Table - continued from previous page

\begin{tabular}{|c|c|c|c|c|c|}
\hline \multirow[t]{2}{*}{ name } & \multirow[t]{2}{*}{ SI-Symbol } & \multirow{2}{*}{$\begin{array}{r}\text { number of } \\
\text { points }\end{array}$} & \multirow[t]{2}{*}{$s_{\text {crit }}^{*}$} & \multirow{2}{*}{$\begin{array}{r}\text { AAD / \% } \\
\text { full GC method }\end{array}$} & \multirow{2}{*}{$\begin{array}{r}\text { AAD / \% } \\
\text { individual method }\end{array}$} \\
\hline & & & & & \\
\hline Hexanoic acid butyl ester & H-42 & 144 & -0.148 & 2.445 & - \\
\hline Hexanoic acid pentyl ester & H-43 & 151 & -0.154 & 2.149 & - \\
\hline Hexanoic acid hexyl ester & $\mathrm{H}-44$ & 147 & -0.160 & 2.064 & - \\
\hline Hexanoic acid heptyl ester & H- 45 & 147 & -0.166 & 2.082 & - \\
\hline Hexanoic acid octyl ester & H-46 & 125 & -0.171 & 2.695 & - \\
\hline Hexanoic acid nonyl ester & $\mathrm{H}-47$ & 132 & -0.177 & 3.443 & - \\
\hline Octanoic acid methyl ester & $\mathrm{H}-48$ & 247 & -0.141 & 15.340 & - \\
\hline Hexadecanoic acid butyl ester & H-49 & 9 & -0.204 & 5.460 & - \\
\hline Octadecanoic acid methyl ester & $\mathrm{H}-50$ & 8 & -0.199 & 9.293 & - \\
\hline \multicolumn{6}{|c|}{ Alcohols } \\
\hline Methanol & $\mathrm{K}-1$ & 931 & -0.684 & 11.851 & 6.995 \\
\hline Ethanol & $\mathrm{K}-2$ & 876 & -0.388 & 8.456 & 6.729 \\
\hline 1-Propanol & $\mathrm{K}-3$ & 402 & -0.372 & 7.662 & 8.017 \\
\hline 2-Propanol & $\mathrm{K}-4$ & 396 & -0.369 & 4.643 & 10.529 \\
\hline 1-Butanol & $\mathrm{K}-5$ & 251 & -0.367 & 5.319 & 4.630 \\
\hline 2-Butanol & $\mathrm{K}-6$ & 76 & -0.363 & 4.251 & 2.083 \\
\hline 1-Pentanol & $\mathrm{K}-7$ & 132 & -0.372 & 4.028 & 2.686 \\
\hline 2-Pentanol & $\mathrm{K}-8$ & 55 & -0.367 & 2.874 & 13.248 \\
\hline 1-Hexanol & K-9 & 256 & -0.382 & 4.047 & 2.447 \\
\hline 1-Heptanol & $\mathrm{K}-10$ & 267 & -0.396 & 4.745 & 3.481 \\
\hline 1-Octanol & $\mathrm{K}-11$ & 211 & -0.412 & 5.550 & 3.295 \\
\hline 2-Octanol & $\mathrm{K}-12$ & 36 & -0.405 & 5.847 & 1.779 \\
\hline 1-Nonanol & $\mathrm{K}-13$ & 182 & -0.429 & 4.025 & 2.161 \\
\hline 1-Decanol & $\mathrm{K}-14$ & 286 & -0.447 & 5.630 & 3.990 \\
\hline 1-Undecanol & $\mathrm{K}-15$ & 92 & -0.465 & 3.257 & 2.069 \\
\hline 1-Dodecanol & $\mathrm{K}-16$ & 94 & -0.483 & 6.993 & 2.081 \\
\hline 1-Tetradecanol & $\mathrm{K}-17$ & 14 & -0.518 & 5.680 & 1.098 \\
\hline 1-Hexadecanol & $\mathrm{K}-18$ & 4 & -0.553 & 9.026 & 1.073 \\
\hline 1-Octadecanol & $\mathrm{K}-19$ & 13 & -0.587 & 3.075 & 1.188 \\
\hline
\end{tabular}


Table - continued from previous page

\begin{tabular}{|c|c|c|c|c|c|}
\hline \multirow[t]{2}{*}{ name } & \multirow[t]{2}{*}{ SI-Symbol } & \multirow{2}{*}{$\begin{array}{r}\text { number of } \\
\text { points }\end{array}$} & \multirow[t]{2}{*}{$s_{\text {crit }}^{*}$} & \multirow{2}{*}{$\begin{array}{r}\text { AAD / \% } \\
\text { full GC method }\end{array}$} & \multirow{2}{*}{$\begin{array}{r}\text { AAD / \% } \\
\text { individual method }\end{array}$} \\
\hline & & & & & \\
\hline 2-Methyl-2-propanol & K-20 & 94 & -0.336 & 26.264 & 3.958 \\
\hline 2-Methyl-1-propanol & $\mathrm{K}-21$ & 43 & -0.363 & 4.701 & 4.974 \\
\hline 2-Methyl-1-butanol & K-22 & 27 & -0.367 & 11.485 & 2.674 \\
\hline 3-Methyl-1-butanol & $\mathrm{K}-23$ & 64 & -0.367 & 5.599 & 9.162 \\
\hline 3,3-Dimethyl-2-butanol & K-24 & 8 & -0.340 & 8.815 & - \\
\hline 2-Methyl-2-pentanol & $\mathrm{K}-25$ & 42 & -0.346 & 8.664 & - \\
\hline 2,3-Dimethyl-2-butanol & K-26 & 36 & -0.340 & 6.481 & - \\
\hline 2-Methyl-2-hexanol & $\mathrm{K}-27$ & 35 & -0.359 & 9.070 & - \\
\hline 5-Methyl-1-hexanol & $\mathrm{K}-28$ & 11 & -0.390 & 4.967 & - \\
\hline \multicolumn{6}{|c|}{ Ethers } \\
\hline Dimethyl ether & L-1 & 347 & -0.229 & 5.089 & 4.009 \\
\hline Methyl propyl ether & L-2 & 146 & -0.268 & 7.587 & 5.834 \\
\hline Diethyl ether & L-3 & 210 & -0.257 & 7.072 & 5.548 \\
\hline Methyl tert-butyl ether & L-4 & 7 & -0.252 & 2.944 & 0.074 \\
\hline Di-n-propyl ether & L-5 & 142 & -0.291 & 6.827 & 2.412 \\
\hline Ethyl butyl ether & L-6 & 9 & -0.291 & 9.116 & 2.459 \\
\hline Dibutyl ether & L-7 & 262 & -0.325 & 4.873 & 4.695 \\
\hline Dipentyl ether & L-8 & 242 & -0.358 & 7.046 & 5.138 \\
\hline Diphenyl ether & L-9 & 37 & -0.336 & 2.497 & 2.020 \\
\hline Propyl butyl ether & L-10 & 2 & -0.308 & 16.344 & - \\
\hline Diisopentyl ether & L-11 & 8 & -0.347 & 3.912 & - \\
\hline Dihexyl ether & L-12 & 101 & -0.389 & 9.621 & - \\
\hline Diheptylether & L-13 & 9 & -0.418 & 8.306 & - \\
\hline Di-n-octyl ether & L-14 & 74 & -0.447 & 9.646 & - \\
\hline Eugenol & $\mathrm{L}-15$ & 7 & -0.330 & 4.515 & - \\
\hline \multicolumn{6}{|c|}{ contains Nitrogen } \\
\hline Methylamine & M-1 & 131 & -0.240 & 6.618 & 4.282 \\
\hline Isopropylamine & M-2 & 5 & -0.244 & 3.509 & 0.439 \\
\hline N,N-Diethylamine & M-3 & 10 & -0.263 & 12.348 & 5.825 \\
\hline
\end{tabular}


Table - continued from previous page

\begin{tabular}{|c|c|c|c|c|c|}
\hline name & SI-Symbol & $\begin{array}{r}\text { number of } \\
\text { points }\end{array}$ & $s_{\text {crit }}^{*}$ & $\begin{array}{r}\mathrm{AAD} / \% \\
\text { full GC method }\end{array}$ & $\begin{array}{r}\text { AAD / \% } \\
\text { individual method }\end{array}$ \\
\hline Hexylamine & M-4 & 4 & -0.306 & 14.524 & 0.958 \\
\hline 1-Octanamine & M-5 & 4 & -0.343 & 13.310 & 1.479 \\
\hline Dibutylamine & M-6 & 4 & -0.338 & 6.657 & 1.143 \\
\hline Diisopropylamine & M-7 & 4 & -0.286 & 5.263 & - \\
\hline \multicolumn{6}{|c|}{ Others } \\
\hline Ethyne & $\mathrm{N}-1$ & 29 & -0.893 & 6.655 & 10.705 \\
\hline Water & $\mathrm{N}-2$ & 2923 & -0.880 & 5.735 & 6.015 \\
\hline Carbon dioxide & $\mathrm{N}-3$ & 1460 & -0.861 & 5.972 & 9.307 \\
\hline \multicolumn{6}{|c|}{ multiple functional groups } \\
\hline 1,2-Ethanediol & O-1 & 175 & -1.434 & 27.146 & 2.480 \\
\hline 1,3-Propanediol & $\mathrm{O}-2$ & 10 & -1.468 & 12.759 & 0.250 \\
\hline 1,2-Propanediol & $\mathrm{O}-3$ & 51 & -1.441 & 16.436 & 2.942 \\
\hline 1,3-Butanediol & $\mathrm{O}-4$ & 15 & -1.495 & 11.136 & 10.025 \\
\hline 1,4-Butanediol & O-5 & 26 & -1.525 & 9.563 & 2.496 \\
\hline 2,2-Dimethyl-1,3-propanediol & O-6 & 5 & -1.416 & 18.795 & - \\
\hline 1,5-Pentanediol & O-7 & 7 & -1.596 & 3.396 & 0.612 \\
\hline 1,6-Hexanediol & $\mathrm{O}-8$ & 7 & -1.674 & 1.825 & 0.129 \\
\hline 1,8-Octanediol & O-9 & 8 & -1.839 & 5.834 & - \\
\hline 1,9-Nonanediol & $\mathrm{O}-10$ & 7 & -1.923 & 7.974 & - \\
\hline 1,10-Decanediol & $\mathrm{O}-11$ & 7 & -2.006 & 11.680 & 0.258 \\
\hline Glycerol & $\mathrm{O}-12$ & 164 & -1.392 & 33.983 & 2.204 \\
\hline 2-(Hydroxymethyl)-2-methyl-1,3-propanediol & $\mathrm{O}-13$ & 1 & -1.416 & 27.779 & - \\
\hline 2-Ethyl-2-hydroxymethyl-1,3-propanediol & $\mathrm{O}-14$ & 3 & -1.511 & 2.679 & - \\
\hline Biphenyl & O-15 & 37 & -1.043 & 27.326 & 2.671 \\
\hline Diphenylmethane & $\mathrm{O}-16$ & 26 & -1.192 & 12.489 & 0.658 \\
\hline Ditolylmethane & O-17 & 53 & -1.395 & 10.508 & - \\
\hline Isopropylbiphenyl & $\mathrm{O}-18$ & 24 & -1.390 & 11.925 & - \\
\hline Bis (isopropylphenyl) methane & $\mathrm{O}-19$ & 37 & -1.808 & 11.981 & - \\
\hline O-Terphenyl & O-20 & 28 & -1.621 & 11.753 & 1.268 \\
\hline
\end{tabular}


Table - continued from previous page

\begin{tabular}{|c|c|c|c|c|c|}
\hline name & SI-Symbol & & $s_{\text {crit }}^{*}$ & $\mathrm{AAD} / \%$ & $\mathrm{AAD} / \%$ \\
\hline & & points & & full GC method & individual method \\
\hline m-Terphenyl & $\mathrm{O}-21$ & 22 & -1.621 & 22.234 & 1.085 \\
\hline p-Terphenyl & $\mathrm{O}-22$ & 12 & -1.621 & 28.730 & 2.486 \\
\hline Bicyclopentyl & O-23 & 4 & -0.859 & 6.091 & - \\
\hline Bicyclohexyl & $\mathrm{O}-24$ & 4 & -1.014 & 23.690 & 0.164 \\
\hline Dicyclohexylmethane & $\mathrm{O}-25$ & 6 & -1.155 & 25.892 & - \\
\hline 1,1-Dicyclohexylheptane & O-26 & 5 & -1.842 & 17.854 & - \\
\hline Diethyl succinate & $\mathrm{O}-27$ & 256 & -1.340 & 27.520 & 5.331 \\
\hline Dipropyl succinate & O-28 & 171 & -1.616 & 30.384 & - \\
\hline Decanedioic acid dibutyl ester & O-29 & 73 & -2.488 & 21.035 & 1.509 \\
\hline Di-n-hexyladipate & $\mathrm{O}-30$ & 159 & -2.488 & 15.690 & - \\
\hline Diisobutyl sebacate & $\mathrm{O}-31$ & 201 & -2.429 & 24.320 & - \\
\hline Di-n-heptyl adipate & $\mathrm{O}-32$ & 173 & -2.669 & 14.467 & - \\
\hline Di(2-ethylhexyl) adipate & O-33 & 4 & -2.787 & 4.920 & 1.725 \\
\hline Hexanedioic acid dioctyl ester & $\mathrm{O}-34$ & 186 & -2.840 & 13.839 & - \\
\hline Succinic acid dinonyl ester & O-35 & 180 & -2.840 & 15.150 & - \\
\hline Dimethoxymethane & O-36 & 185 & -0.499 & 30.990 & 1.473 \\
\hline 1,2-Dimethoxyethane & O-37 & 165 & -0.711 & 30.870 & 0.968 \\
\hline 1,2-Diethoxyethane & O-38 & 7 & -0.903 & 23.761 & 0.494 \\
\hline Dibutoxymethane & O-39 & 10 & -1.358 & 8.405 & - \\
\hline Ethylene glycol di-n-butyl ether & $\mathrm{O}-40$ & 6 & -1.492 & 11.375 & - \\
\hline Diethylene glycol dimethyl ether & $\mathrm{O}-41$ & 14 & -1.230 & 13.964 & 1.540 \\
\hline Diethylene glycol diethyl ether & $\mathrm{O}-42$ & 43 & -1.367 & 15.787 & - \\
\hline Diethylene glycol dibutyl ether & $\mathrm{O}-43$ & 42 & -1.876 & 6.156 & - \\
\hline Triethylene glycol dimethyl ether & $\mathrm{O}-44$ & 5 & -1.666 & 28.516 & - \\
\hline Tetraethylene glycol dimethyl ether & O-45 & 4 & -2.046 & 26.780 & 8.541 \\
\hline mixed & ional group & s, each gro & up only & once & \\
\hline 3,7,11,15-Tetramethyl-1-hexadecen-3-ol & $\mathrm{P}-1$ & 6 & -0.846 & 27.863 & - \\
\hline Phenol & $\mathrm{P}-2$ & 22 & -0.511 & 4.675 & 0.659 \\
\hline Benzyl alcohol & $\mathrm{P}-3$ & 4 & -0.537 & 1.578 & 0.178 \\
\hline
\end{tabular}


Table - continued from previous page

\begin{tabular}{|c|c|c|c|c|c|}
\hline name & SI-Symbol & $\begin{array}{r}\text { number of } \\
\text { points }\end{array}$ & $s_{\text {crit }}^{*}$ & $\begin{array}{r}\mathrm{AAD} / \% \\
\text { full GC method }\end{array}$ & $\begin{array}{r}\text { AAD / \% } \\
\text { individual method }\end{array}$ \\
\hline 3-Methylphenol & $\mathrm{P}-4$ & 11 & -0.526 & 10.615 & 0.526 \\
\hline Cyclopentanol & $\mathrm{P}-5$ & 5 & -0.504 & 20.363 & - \\
\hline Cyclohexanol & $\mathrm{P}-6$ & 15 & -0.495 & 44.295 & 0.817 \\
\hline 2-Methoxyethanol & $\mathrm{P}-7$ & 10 & -0.586 & 10.086 & 3.123 \\
\hline 2-Ethoxyethanol & $\mathrm{P}-8$ & 45 & -0.581 & 14.440 & 1.072 \\
\hline 2-Butoxyethanol & P-9 & 50 & -0.613 & 6.812 & 5.082 \\
\hline Diethylene glycol monomethyl ether & $\mathrm{P}-10$ & 43 & -0.651 & 8.152 & 5.026 \\
\hline 2-Phenoxy-ethanol & $\mathrm{P}-11$ & 6 & -0.611 & 14.038 & - \\
\hline Ethylene glycol monohexyl ether & $\mathrm{P}-12$ & 6 & -0.658 & 4.432 & 0.182 \\
\hline 2-Ethylpropenal & $\mathrm{P}-13$ & 5 & -0.116 & 79.692 & - \\
\hline Benzaldehyde & $\mathrm{P}-14$ & 9 & -0.266 & 13.819 & 0.744 \\
\hline Cyclopentene & $\mathrm{P}-15$ & 15 & -0.125 & 70.791 & 2.746 \\
\hline Cyclohexene & $\mathrm{P}-16$ & 76 & -0.170 & 62.046 & 2.192 \\
\hline 1-Methylcyclohexene & $\mathrm{P}-17$ & 4 & -0.292 & 49.234 & - \\
\hline Styrene & $\mathrm{P}-18$ & 14 & -0.523 & 26.925 & 0.242 \\
\hline Amyl salicylate & $\mathrm{P}-19$ & 69 & -0.735 & 11.492 & - \\
\hline Vinyl acetate & $\mathrm{P}-20$ & 5 & -0.485 & 4.261 & 1.026 \\
\hline trans-2-Butenoic acid methyl ester & $\mathrm{P}-21$ & 70 & -0.543 & 25.739 & - \\
\hline Methacrylic acid methyl ester & $\mathrm{P}-22$ & 8 & -0.551 & 8.761 & 1.286 \\
\hline trans-2-Butenoic acid ethyl ester & P-23 & 71 & -0.566 & 69.476 & - \\
\hline Methyl oleate & $\mathrm{P}-24$ & 395 & -0.947 & 13.647 & - \\
\hline Benzoic acid methyl ester & $\mathrm{P}-25$ & 137 & -0.562 & 62.980 & 0.871 \\
\hline Ethyl benzoate & P-26 & 277 & -0.615 & 2.187 & 1.394 \\
\hline Salicylic acid methyl ester & $\mathrm{P}-27$ & 66 & -0.623 & 10.788 & 2.633 \\
\hline Propyl benzoate & $\mathrm{P}-28$ & 141 & -0.649 & 3.533 & 1.117 \\
\hline Carbonic acid dimethyl ester & P-29 & 19 & -0.531 & 12.060 & 0.439 \\
\hline Ethylene glycol acetate monomethyl ether & P-30 & 127 & -0.567 & 68.180 & - \\
\hline Carbonic acid diethyl ester & P-31 & 140 & -0.567 & 64.960 & 1.031 \\
\hline Ethyleneglycol mono(2-ethylbutyl) ether & P-32 & 6 & -0.649 & 9.500 & - \\
\hline
\end{tabular}


Table - continued from previous page

\begin{tabular}{|c|c|c|c|c|c|}
\hline name & SI-Symbol & & $s_{\text {crit }}^{*}$ & $\mathrm{AAD} / \%$ & $\mathrm{AAD} / \%$ \\
\hline & & points & & full GC method & individual method \\
\hline 2-Hydroxyacetophenone & $\mathrm{P}-33$ & 8 & -0.603 & 69.557 & 5.858 \\
\hline Acetophenone & $\mathrm{P}-34$ & 15 & -0.342 & 39.677 & 18.951 \\
\hline p-Methylacetophenone & $\mathrm{P}-35$ & 8 & -0.382 & 66.760 & - \\
\hline Cyclopentanone & P-36 & 18 & -0.215 & 18.195 & 3.394 \\
\hline Cyclohexanone & P-37 & 7 & -0.247 & 41.884 & 5.152 \\
\hline Acetic anhydride & P-38 & 6 & -0.272 & 27.010 & 12.500 \\
\hline Monoethanolamine & P-39 & 18 & -0.403 & 68.793 & 7.685 \\
\hline Formamide & $\mathrm{P}-40$ & 8 & -0.457 & 17.826 & 1.081 \\
\hline Aniline & $\mathrm{P}-41$ & 24 & -0.473 & 20.792 & 4.336 \\
\hline Piperidine & $\mathrm{P}-42$ & 2 & -0.430 & 32.470 & - \\
\hline Cyclohexylamine & $\mathrm{P}-43$ & 5 & -0.453 & 10.384 & 4.741 \\
\hline mixed function & al groups, & ach group 1 & $\operatorname{maximu}$ & m twice & \\
\hline 2-Phenylphenol & Q-1 & 5 & -0.495 & 5.734 & - \\
\hline Diethylene glycol & $\mathrm{Q}-2$ & 87 & -0.444 & 5.424 & 1.348 \\
\hline Diethylene glycol ethyl ether & $\mathrm{Q}-3$ & 7 & -0.518 & 15.131 & 4.786 \\
\hline Triethylene glycol & $\mathrm{Q}-4$ & 116 & -0.518 & 3.504 & 1.078 \\
\hline Diethylene glycol monobutyl ether & $\mathrm{Q}-1$ & 7 & -0.554 & 8.994 & 0.338 \\
\hline 2,2'-Diethanolamine (DEA) & $\mathrm{Q}-2$ & 13 & -0.379 & 41.851 & 0.471 \\
\hline (Z)-2-Butenedioic acid dihexyl ester & Q-3 & 128 & -0.738 & 14.705 & - \\
\hline (Z,Z)-9,12-Octadecadienoic acid methyl ester & Q-4 & 350 & -0.760 & 21.513 & - \\
\hline Diheptyl maleate & Q-1 & 131 & -0.776 & 15.204 & - \\
\hline Dioctyl maleate & $\mathrm{Q}-2$ & 129 & -0.813 & 12.553 & - \\
\hline (Z)-2-Butenedioic acid dinonyl ester & Q-3 & 264 & -0.849 & 12.414 & - \\
\hline Dimethyl terephthalate & $\mathrm{Q}-4$ & 22 & -0.567 & 16.081 & 0.810 \\
\hline Phthalic acid dimethyl ester & Q-1 & 133 & -0.567 & 15.169 & 2.582 \\
\hline Phthalic acid diethyl ester & Q-2 & 30 & -0.612 & 14.434 & 2.921 \\
\hline Phthalic acid dipropyl ester & Q-3 & 29 & -0.655 & 23.968 & 1.378 \\
\hline Phthalic acid diisopropyl ester & $\mathrm{Q}-4$ & 16 & -0.640 & 8.795 & - \\
\hline Phthalic acid dibutyl ester & Q-1 & 42 & -0.696 & 12.647 & 4.043 \\
\hline
\end{tabular}


Table - continued from previous page

\begin{tabular}{|c|c|c|c|c|c|}
\hline name & SI-Symbol & $\begin{array}{r}\text { number of } \\
\text { points }\end{array}$ & $s_{\text {crit }}^{*}$ & $\begin{array}{r}\mathrm{AAD} / \% \\
\text { full GC method }\end{array}$ & $\begin{array}{r}\text { AAD / \% } \\
\text { individual method }\end{array}$ \\
\hline Diisobutyl terephthalate & Q-2 & 17 & -0.682 & 11.250 & - \\
\hline Terephthalic acid dibutyl ester & Q-3 & 29 & -0.696 & 8.194 & - \\
\hline Phthalic acid diisobutyl ester & Q-4 & 16 & -0.682 & 5.831 & - \\
\hline Di-n-hexylphthalate & Q-1 & 17 & -0.775 & 26.382 & 4.301 \\
\hline Phthalic acid diheptyl ester & $\mathrm{Q}-2$ & 20 & -0.812 & 15.654 & - \\
\hline Bis(2-ethylhexyl) phthalate & Q-3 & 16 & -0.835 & 4.792 & 0.349 \\
\hline Phthalic acid dioctyl ester & Q-4 & 23 & -0.848 & 11.689 & - \\
\hline Diallyl ether & Q-1 & 89 & -0.452 & 65.765 & - \\
\hline 1,3-Benzenedimethanamine & Q-2 & 4 & -0.435 & 56.063 & - \\
\hline 1,3-Cyclopentadiene & Q-3 & 5 & -0.355 & 84.320 & 0.702 \\
\hline 1,3-Cyclohexadiene & $\mathrm{Q}-4$ & 18 & -0.379 & 77.387 & 0.238 \\
\hline 1,4-Cyclohexadiene & Q-1 & 18 & -0.379 & 79.306 & 0.263 \\
\hline \multicolumn{6}{|c|}{ mixed functional groups, some groups three times or more } \\
\hline Tetraethylene glycol & $\mathrm{R}-1$ & 67 & -0.211 & 8.192 & 3.159 \\
\hline Pentaethylene glycol & $\mathrm{R}-2$ & 30 & -0.236 & 11.054 & - \\
\hline Hexaethyleneglycol & $\mathrm{R}-3$ & 32 & -0.261 & 14.419 & - \\
\hline Heptaethyleneglycol & $\mathrm{R}-4$ & 26 & -0.284 & 16.598 & - \\
\hline 6,10-Dimethyl-3,5,9-undecatrien-2-one & $\mathrm{R}-5$ & 6 & -0.239 & 20.214 & - \\
\hline \multicolumn{6}{|c|}{ segments missing in GC PCP-SAFT } \\
\hline Diisopropyl ether & $\mathrm{S}-1$ & 143 & & - & 1.347 \\
\hline Triethylamine & $\mathrm{S}-2$ & 20 & & - & 3.375 \\
\hline Hydrazine & $\mathrm{S}-3$ & 77 & & - & 3.085 \\
\hline 1-Nitropropane & S-4 & 4 & & - & 0.823 \\
\hline Nitromethane & S-5 & 15 & & - & 2.486 \\
\hline Nitroethane & S-6 & 165 & & - & 3.900 \\
\hline 2-Nitropropane & $\mathrm{S}-7$ & 14 & & - & 2.267 \\
\hline Nitrobenzene & S-8 & 40 & & - & 6.832 \\
\hline m-Nitrotoluene & S-9 & 12 & & - & 0.219 \\
\hline Pyridine & S-10 & 9 & & - & 1.036 \\
\hline
\end{tabular}


Table - continued from previous page

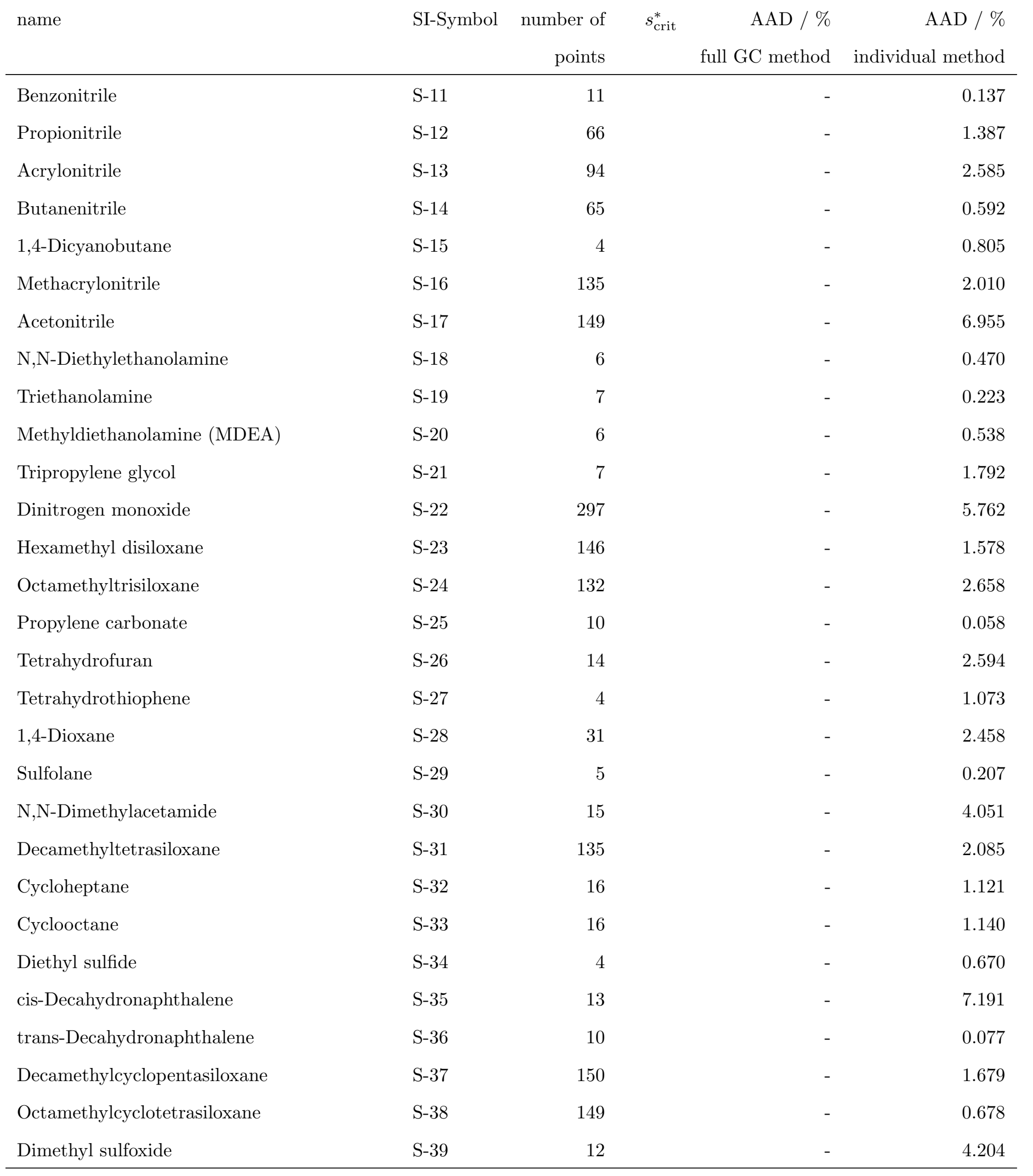


Table - continued from previous page

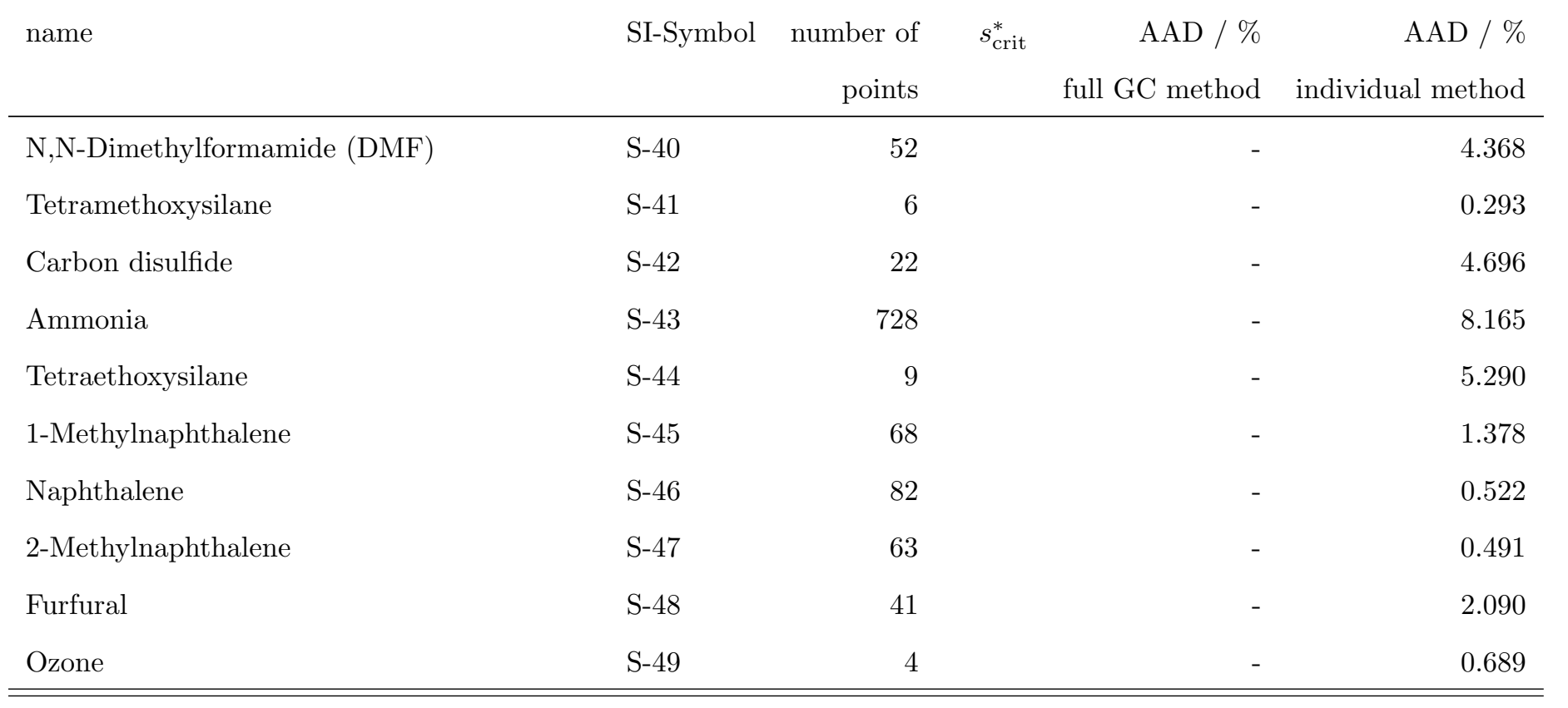




\section{Parameters $(A-D)$ for individual Entropy-Scaling with PCP-SAFT}

Correlation parameters $A-C$ for individual entropy scaling for all components, for which no parameters were published yet. $D$ is set to zero for all substances, since its influence is neglectable in the individual approach.

Table S5:

\begin{tabular}{|c|c|c|c|}
\hline Name & A & B & $\mathrm{C}$ \\
\hline \multicolumn{4}{|c|}{ Alkenes } \\
\hline Isobutylene & 0.07483 & 1.55986 & 4.42680 \\
\hline 2,3-Dimethyl-1-butene & 0.0 & -0.64795 & 0.99169 \\
\hline 2,3-Dimethyl-2-butene & 0.0 & -0.66721 & 0.94891 \\
\hline 2-Heptene & 0.0 & -0.60426 & 1.27720 \\
\hline 2-Methylbuta-1,3-diene & -0.05766 & -0.46053 & 1.56562 \\
\hline \multicolumn{4}{|c|}{ Cyclic Alkanes } \\
\hline Bicyclohexyl & 0.0 & -0.21067 & 2.26358 \\
\hline Ethylcyclohexane & 0.07477 & -0.58273 & 1.11457 \\
\hline Propylcyclohexane & 0.26614 & -0.52649 & 1.01660 \\
\hline cis-1,2-Dimethylcyclohexane & 0.0 & -0.61586 & 1.09147 \\
\hline cis-1,3-Dimethyl-cyclohexane & 0.0 & -0.49817 & 1.37660 \\
\hline trans-1,2-Dimethylcyclohexane & 0.0 & -0.48908 & 1.44026 \\
\hline trans-1,4-Dimethylcyclohexane & 0.0 & -0.49929 & 1.40414 \\
\hline \multicolumn{4}{|c|}{ Aromatics } \\
\hline Diphenylmethane & 0.0 & -0.36671 & 1.80936 \\
\hline 1,2-Diethylbenzene & 0.0 & -0.55567 & 1.27650 \\
\hline
\end{tabular}


Table - continued from previous page

\begin{tabular}{|c|c|c|c|}
\hline Name & A & B & $\mathrm{C}$ \\
\hline m-Terphenyl & 0.35096 & -0.34288 & 1.50011 \\
\hline p-Terphenyl & 0.36378 & -0.54675 & 1.04831 \\
\hline Biphenyl & 0.19102 & -0.48255 & 1.30469 \\
\hline \multicolumn{4}{|c|}{ Esters } \\
\hline Di(2-ethylhexyl)adipate & 0.0 & -0.50860 & 2.41211 \\
\hline Ethylbutyrate & 0.15395 & -0.61105 & 1.04292 \\
\hline Methylformate & -0.55994 & -0.30040 & 2.39540 \\
\hline Decanedioicaciddibutylester & 0.0 & -0.47736 & 1.82906 \\
\hline Formicacidethylester & -0.04841 & -0.48800 & 1.33416 \\
\hline Formicacidpropylester & 0.03880 & -0.59708 & 0.98757 \\
\hline Heptylformate & 0.0 & -0.78237 & -0.05382 \\
\hline Formicacidoctylester & 0.51843 & -0.56844 & 1.06385 \\
\hline Aceticacid3-methylbutylester & 0.32853 & -0.82454 & 0.14052 \\
\hline n-Butyloctadecanoate & 0.0 & -0.21795 & 2.37847 \\
\hline Methylpropanoate & -0.31767 & -0.20512 & 2.26115 \\
\hline Propanoicacidbutylester & -0.14575 & -0.36734 & 1.80521 \\
\hline Formicacidbutylester & 0.08838 & -0.48238 & 1.26000 \\
\hline Formicacidpentylester & 0.10577 & -0.62602 & 0.99201 \\
\hline \multicolumn{4}{|c|}{ Alcohols } \\
\hline 2-Butanol & -0.57052 & -0.19717 & 1.79621 \\
\hline 2-Octanol & -0.25125 & -0.33457 & 1.62121 \\
\hline 3-Methyl-1-butanol & -0.39999 & -0.43079 & 1.30483 \\
\hline 2-Methyl-1-butanol & -0.45581 & -0.34331 & 1.43463 \\
\hline 2-Methyl-1-propanol & 0.0 & -0.46590 & 0.48784 \\
\hline
\end{tabular}

Continued on next page 
Table - continued from previous page

\begin{tabular}{lrrr} 
Name & A & B & C \\
\hline 1,5-Pentanediol & 0.0 & -0.87989 & -0.29278 \\
1,6-Hexanediol & 0.0 & -0.45765 & 1.03512 \\
1,10-Decanediol & 0.0 & -0.32088 & 1.61150 \\
\hline
\end{tabular}

Ethers

\begin{tabular}{lrrr}
\hline Diethyleneglycol-dimethyl-ether & -0.15647 & -0.70176 & 1.19390 \\
Tetraethylene-glycol-dimethyl-ether & 0.0 & -0.32949 & 2.95300 \\
1,2-Diethoxyethane & 0.0 & -0.58180 & 1.24999 \\
\hline \multicolumn{1}{c}{ Substances Containing } & Nitrogen & \\
\hline N,N-Diethylamine & -0.01033 & -0.33882 & 1.83741 \\
Hexylamine & 0.0 & -0.30369 & 1.88478 \\
1-Octanamine & 0.0 & -0.34512 & 1.93194 \\
Dibutylamine & 0.0 & -0.43847 & 1.60757 \\
Isopropylamine & 0.0 & -0.33714 & 1.49995 \\
Hydrazine & 0.0 & -0.42140 & -1.14552 \\
1-Nitropropane & 0.0 & -0.52611 & 1.15864 \\
Nitroethane & 0.0 & -0.72350 & 0.43799 \\
2-Nitropropane & 0.0 & -0.70527 & 0.63157 \\
Nitrobenzene & 0.0 & -0.67647 & 0.85533 \\
m-Nitrotoluene & 0.0 & -0.55611 & 1.18943 \\
Benzonitrile & 0.0 & -0.64666 & 0.81844 \\
Propionitrile & 0.0 & -0.43635 & 1.47591 \\
Acrylonitrile & 0.0 & -0.96103 & 0.05925 \\
Butanenitrile & 0.0 & -0.71644 & 0.61715 \\
1,4-Dicyanobutane & 0.0 & & \\
\hline & -0.72209 & -0.85776 & 0.96149 \\
\hline & & & \\
& & 0 & next page \\
\hline
\end{tabular}


Table - continued from previous page

\begin{tabular}{lrrr} 
Name & A & B & C \\
\hline Methacrylonitrile & 0.0 & -0.58559 & 1.16137 \\
\hline \multicolumn{1}{c}{ mixed or multiple functional groups } \\
\hline Styrene & 0.0 & -0.47130 & 1.45773 \\
Benzylalcohol & 0.0 & -0.36037 & 1.26410 \\
2,2'-Diethanolamine(DEA) & 0.0 & -0.36881 & 1.20300 \\
3-Methylphenol & 0.0 & -0.25866 & 1.65310 \\
1,3-Cyclopentadiene & 0.0 & -0.73773 & 0.86798 \\
1,3-Cyclohexadiene & 0.0 & -0.55739 & 1.23206 \\
Cyclohexene & 0.0 & -0.56174 & 1.20478 \\
1,4-Cyclohexadiene & 0.0 & -0.57565 & 1.17366 \\
Cyclohexanol & 0.0 & -0.36784 & 0.93080 \\
Cyclohexylamine & 0.0 & -0.92392 & -3.21031 \\
Phenol & 0.0 & -0.24971 & 1.55312 \\
Dimethoxymethane & 0.0 & -0.70530 & 0.77078 \\
2-Methoxyethanol & 0.0 & -0.02641 & 4.12898 \\
2-Ethoxyethanol & 0.0 & -0.30698 & 1.71812 \\
2-Butoxyethanol & 0.0 & -0.31303 & 1.68689 \\
Bis(2-ethylhexyl)phthalate & 0.0 & -0.28142 & 1.96326 \\
2-Hydroxyacetophenone & 0.0 & 0.68198 & 9.51775 \\
SalicylicAcidMethylEster & 0.0 & -0.20864 & 2.22601 \\
DimethylTerephthalate & 0.0 & -0.39441 & 1.74184 \\
PhthalicAcidDimethylEster & 0.64855 & -0.50552 & 0.69637 \\
PhthalicAcidDipropylEster & 0.73382 & -0.57136 & 0.52599 \\
Monoethanolamine & -0.07430 & 1.93077 \\
\hline & 0 & & \\
& 0.0 &
\end{tabular}

Continued on next page 
Table - continued from previous page

\begin{tabular}{lrrr} 
Name & A & B & C \\
\hline Propylbenzoate & 0.0 & -0.43802 & 1.60054 \\
Diethyleneglycol & 0.0 & -0.17036 & 1.80066 \\
Triethyleneglycol & 0.0 & -0.21463 & 2.03870 \\
Tetraethyleneglycol & 0.0 & -0.62460 & 0.82295 \\
Benzoicacid & 0.0 & -0.42349 & 0.84263 \\
MethacrylicAcidMethylEster & 0.0 & -0.72089 & 0.79990 \\
PhthalicAcidDiethylEster & 0.60034 & -0.43004 & 0.87411 \\
PhthalicAcidDibutylEster & 0.88843 & -0.52239 & 0.44660 \\
Di-n-hexylphthalate & 1.22863 & -0.95217 & -0.88821 \\
BenzoicAcidMethylEster & 0.0 & -0.49896 & 1.32812 \\
EthylBenzoate & 0.0 & -0.44218 & 1.54692 \\
CarbonicAcidDimethylEster & 0.0 & -0.68107 & 0.88489 \\
CarbonicAcidDiethylEster & 0.0 & -0.57309 & 1.17265 \\
DiethyleneGlycolMonomethylEther & 0.0 & -0.28079 & 1.90593 \\
DiethyleneGlycolEthylEther & 0.0 & -0.36247 & 1.68142 \\
EthyleneGlycolMonohexylEther & 0.0 & -0.30912 & 2.08880 \\
DiethyleneGlycolMonobutylEther & 0.0 & -0.25675 & 1.93055 \\
AceticAnhydride & 0.0 & -0.54045 & 1.22262 \\
N,N-Diethylethanolamine & 0.0 & -0.35543 & 1.84286 \\
Triethanolamine & 0.0 & -0.28919 & 1.63469 \\
Methyldiethanolamine(MDEA) & 0.0 & -0.24657 & 1.72045 \\
Tripropyleneglycol & 0.0 & -1.98013 & -4.21886 \\
\hline & & & \\
\hline Ethyne & -0.62228 & -0.88697 & 0.70596 \\
\hline & & & \\
\hline & others & ontinued on next page \\
\hline
\end{tabular}


Table - continued from previous page

\begin{tabular}{lrrr} 
Name & A & B & C \\
\hline Diethylsuccinate & 0.0 & -0.32907 & 1.83649 \\
Sulfur\# & 0.0 & 0.00011 & 3.32920 \\
Phosphorus\# & 0.0 & 0.89489 & 11.24345 \\
Tetrahydrothiophene & 0.0 & -0.56469 & 1.16793 \\
1,4-Dioxane & -0.16754 & -0.46607 & 1.61009 \\
Sulfolane & 0.0 & -0.71859 & 0.68120 \\
N,N-Dimethylacetamide & 0.0 & -0.78081 & 0.49268 \\
Diethylsulfide & 0.0 & -0.47011 & 1.53832 \\
cis-Decahydronaphthalene & 0.0 & -0.34063 & 1.98674 \\
trans-Decahydronaphthalene & 0.0 & -0.41576 & 1.94955 \\
N,N-Dimethylformamide(DMF) & -0.69680 & -0.25376 & 2.45183 \\
Tetramethoxysilane & 0.0 & -0.38773 & 2.03337 \\
Tetraethoxysilane & 0.0 & -0.10868 & 2.63396 \\
1-Methylnaphthalene & 0.0 & -0.40001 & 1.62722 \\
Naphthalene & 0.0 & -0.48828 & 1.45158 \\
2-Methylnaphthalene & 0.0 & -0.47613 & 1.49981 \\
Furfural & 0.30286 & -0.13491 & 1.80407 \\
Ozone & 0.0 & -0.27412 & 2.21961 \\
\hline \hline & $=0.0:$ & no & data in \\
gas & CEhase;
\end{tabular}




\section{PCP-SAFT Parameters}

Individual PCP-SAFT parameters (not GC PCP-SAFT) for all components, for which no parameters were published yet.

Table S6:

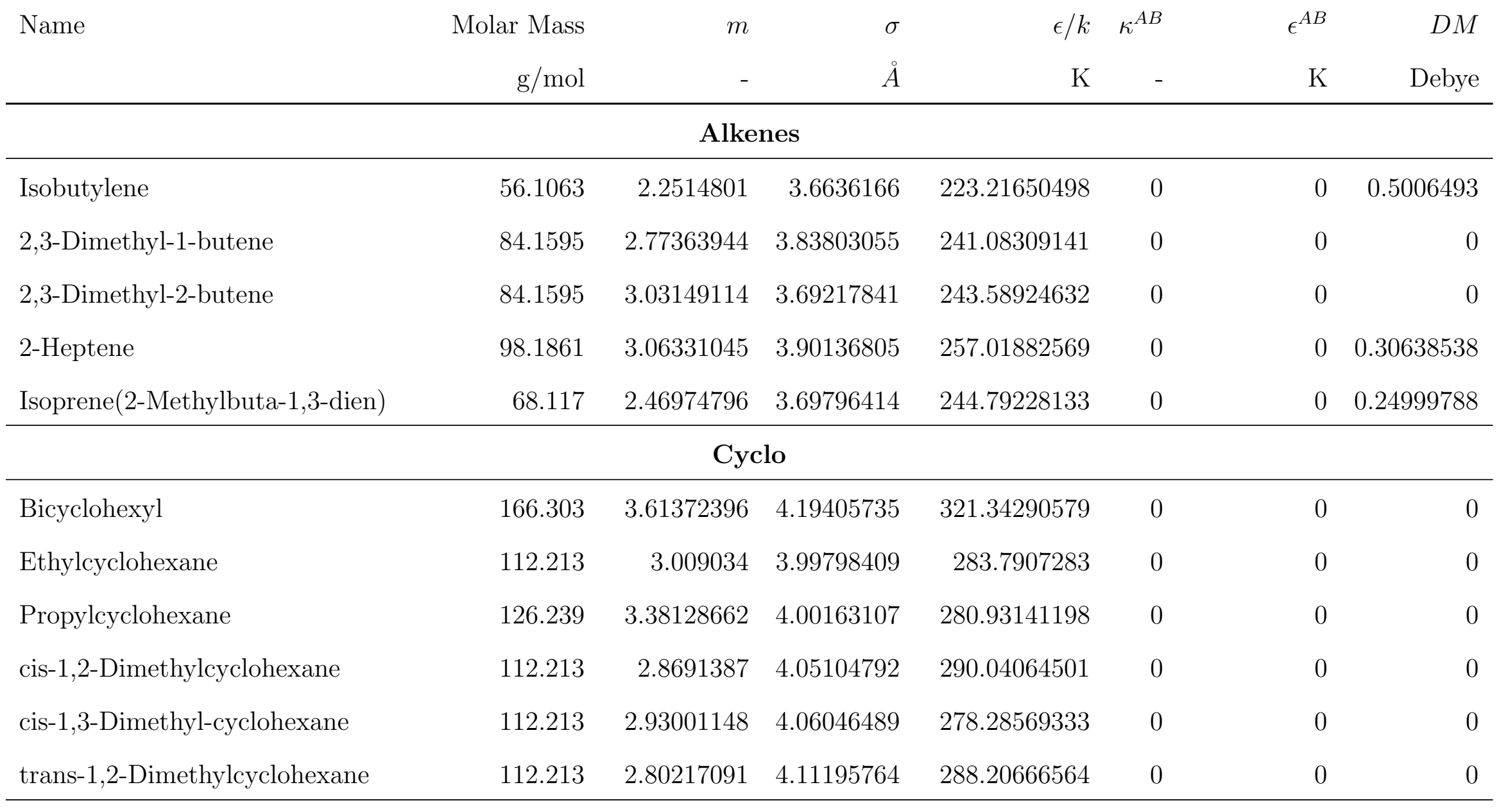


Table - continued from previous page

\begin{tabular}{|c|c|c|c|c|c|c|c|}
\hline \multirow[t]{2}{*}{ Name } & Molar Mass & $m$ & $\sigma$ & $\epsilon / k$ & $\kappa^{A B}$ & $\epsilon^{A B}$ & $D M$ \\
\hline & $\mathrm{g} / \mathrm{mol}$ & - & $\AA$ & $\mathrm{K}$ & - & $\mathrm{K}$ & Debye \\
\hline trans-1,4-Dimethylcyclohexane & 112.213 & 2.8485207 & 4.10613168 & 281.96517035 & 0 & 0 & 0 \\
\hline \multicolumn{8}{|c|}{ Aromatics } \\
\hline Diphenylmethane & 168.234 & 4.23409987 & 3.83678585 & 316.18261029 & 0 & 0 & 0.7704603 \\
\hline 1,2-Diethylbenzene & 134.218 & 3.75757274 & 3.82705757 & 284.55602682 & 0 & 0 & 0.58999571 \\
\hline m-Terphenyl & 230.304 & 5.52509921 & 3.82240602 & 332.3654902 & 0 & 0 & 0.19995993 \\
\hline p-Terphenyl & 230.304 & 5.77865185 & 3.75664023 & 330.44128948 & 0 & 0 & 0.59958 \\
\hline Biphenyl & 154.208 & 3.95976327 & 3.77834207 & 324.36855579 & 0 & 0 & 0 \\
\hline \multicolumn{8}{|c|}{ Esters } \\
\hline Di(2-ethylhexyl)adipate & 370.566 & 3.56405279 & 5.57530614 & 421.97001554 & 0 & 0 & 0.9053658 \\
\hline Ethylbutyrate & 116.158 & 3.45927023 & 3.70591314 & 256.97838446 & 0 & 0 & 1.8107316 \\
\hline Methylformate & 60.052 & 2.65742833 & 3.08961748 & 237.33483908 & 0 & 0 & 1.768761 \\
\hline Decanedioicaciddibutylester & 314.46 & 9.09341665 & 3.72397083 & 251.63179216 & 0 & 0 & 2.4792633 \\
\hline Formicacidethylester & 74.0785 & 2.8658349 & 3.30613454 & 241.08580789 & 0 & 0 & 1.9306476 \\
\hline Formicacidpropylester & 88.1051 & 3.22145209 & 3.40507259 & 243.60394881 & 0 & 0 & 1.9096623 \\
\hline Heptylformate & 144.211 & 0.60817724 & 7.7028527 & 776.6007853 & 0 & 0 & 1.8706896 \\
\hline Formicacidoctylester & 158.238 & 3.29938703 & 4.2537604 & 313.37177763 & 0 & 0 & 1.8736875 \\
\hline
\end{tabular}


Table - continued from previous page

\begin{tabular}{|c|c|c|c|c|c|c|c|}
\hline Name & Molar Mass & $m$ & $\sigma$ & $\epsilon / k$ & $\kappa^{A B}$ & $\epsilon^{A B}$ & $D M$ \\
\hline Aceticacid3-methylbutylester & 130.185 & 4.03482216 & 3.66721468 & 247.41677742 & 0 & 0 & 1.79874 \\
\hline n-Butyloctadecanoate & 340.584 & 10.58845881 & 3.72454132 & 228.30411105 & 0 & 0 & 1.8796833 \\
\hline Methylpropanoate & 88.1051 & 3.43630567 & 3.31449171 & 235.23467086 & 0 & 0 & 1.70310699 \\
\hline Formicacidbutylester & 102.132 & 3.54129976 & 3.48984779 & 245.90599605 & 0 & 0 & 2.0295783 \\
\hline Formicacidpentylester & 116.158 & 3.9795426 & 3.53028854 & 245.90974728 & 0 & 0 & 1.9006686 \\
\hline \multicolumn{8}{|c|}{ Alcohols } \\
\hline 3-Methyl-1-butanol & 88.1482 & 4.37793433 & 3.22224391 & 213.80019455 & 0.03 & 2119.118265 & 1.8497043 \\
\hline 2-Methyl-1-butanol & 88.148 & 4.02143507 & 3.30692459 & 215.91462536 & 0.03 & 2231.564115 & 1.8796833 \\
\hline 2-Methyl-1-propanol & 74.1216 & 4.27710154 & 3.06044221 & 211.92588912 & 0.03 & 1810.490952 & 1.6398513 \\
\hline 1,5-Pentanediol & 104.148 & 4.58496158 & 3.00071149 & 137.99632955 & 0.03 & 5630.302108 & 2.3713389 \\
\hline 1,6-Hexanediol & 118.174 & 4.02505454 & 3.48543691 & 248.15550428 & 0.03 & 3776.660105 & 2.5002486 \\
\hline 1,10-Decanediol & 174.28 & 6.96136504 & 3.36923606 & 256.76352149 & 0.03 & 2452.260779 & 2.1434985 \\
\hline
\end{tabular}


Table - continued from previous page

\begin{tabular}{|c|c|c|c|c|c|c|c|}
\hline Name & Molar Mass & $m$ & $\sigma$ & $\epsilon / k$ & $\kappa^{A B}$ & $\epsilon^{A B}$ & $D M$ \\
\hline \multicolumn{8}{|c|}{ Ethers } \\
\hline Diethyleneglycol-dimethyl-ether & 134.174 & 4.22816112 & 3.57222113 & 258.40783543 & 0 & 0 & 1.96998305 \\
\hline Tetraethylene-glycol-dimethyl-ether & 222.279 & 0.57395438 & 8.95115925 & 1043.08768009 & 0 & 0 & 2.4702696 \\
\hline \multicolumn{8}{|c|}{ Substances Containing Nitrogen } \\
\hline N,N-Diethylamine & 73.1368 & 1.36880311 & 4.72622953 & 295.21357872 & 0.03 & 1547.442233 & 0.9203553 \\
\hline Hexylamine & 101.19 & 3.94342351 & 3.5462531 & 247.46742376 & 0.03 & 82.510822 & 1.588887 \\
\hline Isopropylamine & 59.1103 & 2.43967047 & 3.56086161 & 234.27553689 & 0.03 & 945.013082 & 1.4509836 \\
\hline Hydrazine & 32.0452 & 1.20733663 & 3.43503629 & 368.76910462 & 0.03 & 2073.007202 & 1.7507736 \\
\hline 1-Nitropropane & 89.0932 & 3.02852671 & 3.43398085 & 269.95816973 & 0 & 0 & 3.657438 \\
\hline Nitroethane & 75.0666 & 2.95444798 & 3.21587938 & 252.86240319 & 0 & 0 & 3.657438 \\
\hline 2-Nitropropane & 89.0932 & 2.93864229 & 3.47387916 & 262.93119463 & 0 & 0 & 3.717396 \\
\hline Nitrobenzene & 123.109 & 3.19056277 & 3.59606986 & 318.02757086 & 0 & 0 & 4.227039 \\
\hline m-Nitrotoluene & 137.136 & 3.17444905 & 3.78491342 & 337.14991874 & 0 & 0 & 4.227039 \\
\hline
\end{tabular}


Table - continued from previous page

\begin{tabular}{|c|c|c|c|c|c|c|c|}
\hline \multirow[t]{2}{*}{ Name } & Molar Mass & $m$ & $\sigma$ & $\epsilon / k$ & $\kappa^{A B}$ & $\epsilon^{A B}$ & $D M$ \\
\hline & $\mathrm{g} / \mathrm{mol}$ & - & $\AA$ & $\mathrm{K}$ & - & $\mathrm{K}$ & Debye \\
\hline Benzonitrile & 103.121 & 3.1939634 & 3.57760237 & 302.7820018 & 0 & 0 & 4.167081 \\
\hline Propionitrile & 55.0785 & 2.81089271 & 3.24759465 & 226.58975362 & 0 & 0 & 4.017186 \\
\hline Acrylonitrile & 53.0626 & 2.30326522 & 3.38761506 & 229.02795427 & 0 & 0 & 3.867291 \\
\hline Butanenitrile & 69.1051 & 2.95655209 & 3.43498587 & 249.08315527 & 0 & 0 & 4.06994904 \\
\hline 1,4-Dicyanobutane & 108.141 & 3.3299288 & 3.69309216 & 388.97468328 & 0 & 0 & 3.747375 \\
\hline Methacrylonitrile & 67.0892 & 2.15598833 & 3.76307068 & 275.20795813 & 0 & 0 & 3.687417 \\
\hline \multicolumn{8}{|c|}{ mixed or multiple functional groups } \\
\hline Styrene & 104.149 & 3.131905 & 3.69608667 & 293.27444694 & 0 & 0 & 0.12980907 \\
\hline Benzylalcohol & 108.138 & 3.51285899 & 3.49547545 & 303.81055124 & 0.03 & 1834.407547 & 1.708803 \\
\hline 2,2'-Diethanolamine(DEA) & 105.136 & 3.9598349 & 3.26101631 & 276.63374264 & 0.03 & 3680.50848 & 0.8514036 \\
\hline 3-Methylphenol & 108.138 & 3.17702169 & 3.62964521 & 315.13990196 & 0.03 & 1858.132747 & 1.588887 \\
\hline 1,3-Cyclopentadiene & 66.1011 & 1.8547324 & 3.8645386 & 301.98919274 & 0 & 0 & 0.419706 \\
\hline 1,3-Cyclohexadiene & 80.1277 & 2.42045264 & 3.7445737 & 288.83934228 & 0 & 0 & 0.4406913 \\
\hline Cyclohexene & 82.1436 & 2.53906421 & 3.75108247 & 281.42712214 & 0 & 0 & 0.329769 \\
\hline 1,4-Cyclohexadiene & 80.1277 & 2.65134014 & 3.60816561 & 281.75205483 & 0 & 0 & 0.13010886 \\
\hline Cyclohexanol & 100.159 & 4.49560245 & 3.18261124 & 242.25182955 & 0.03 & 1728.040538 & 1.858698 \\
\hline
\end{tabular}


Table - continued from previous page

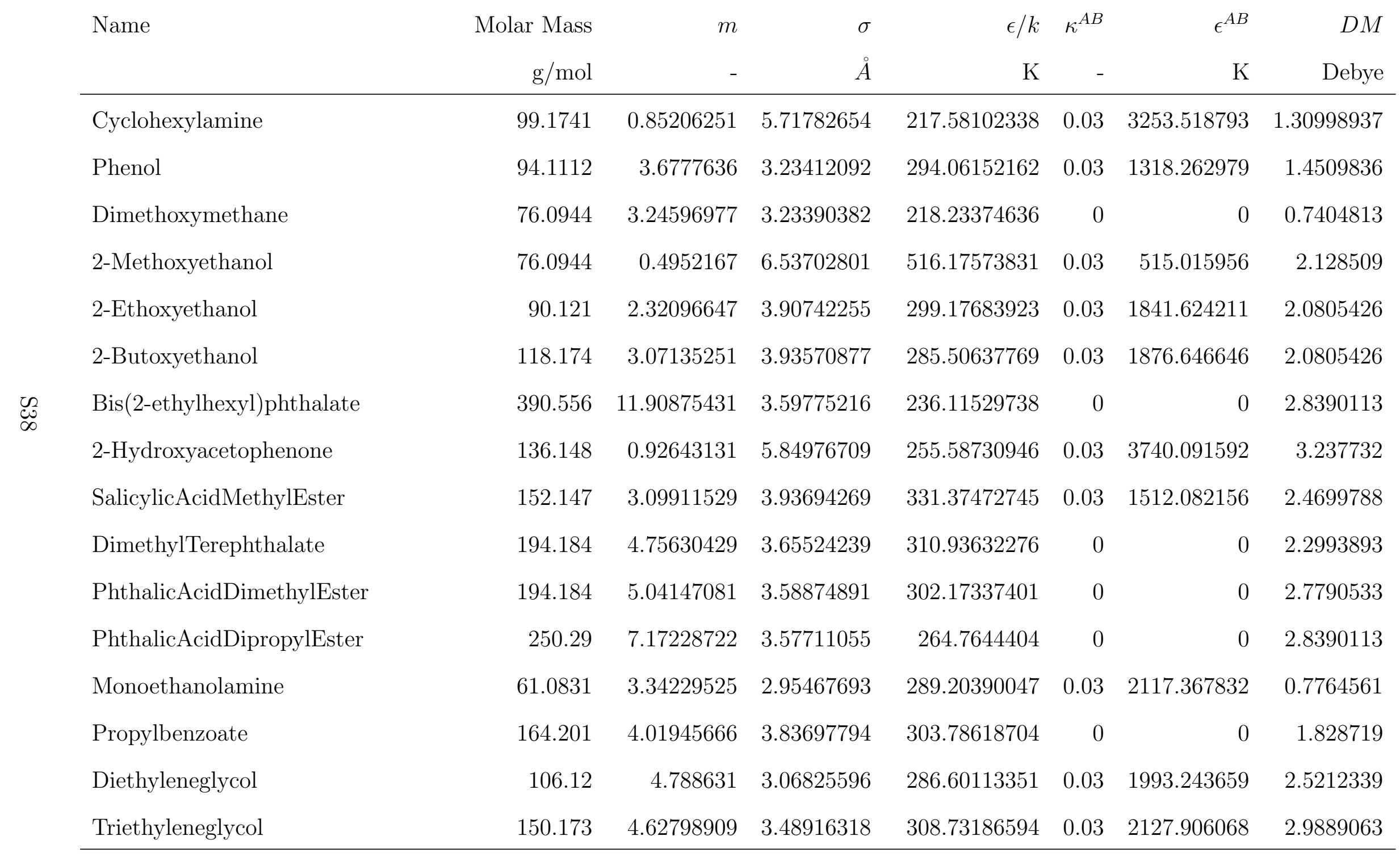


Table - continued from previous page

Name

Molar Mass

$$
m
$$

$\sigma$

$$
\epsilon / k \quad \kappa^{A B}
$$

$\epsilon^{A B}$

$D M$

$\mathrm{g} / \mathrm{mol}$

- $\quad \AA$

$\mathrm{K}$

K Debye

Tetraethyleneglycol

194.226

$5.41866735 \quad 3.46250924$

187.38768182

0.03

5696.270095

3.237732

Benzoicacid

MethacrylicAcidMethylEster

122.121

$3.37273168 \quad 3.55568744$

287.48230113

$0.03 \quad 3363.255554 \quad 1.0012986$

100.116

$3.68291747 \quad 3.35231994$

238.58829744

0

$0 \quad 1.6698303$

PhthalicAcidDiethylEster

PhthalicAcidDibutylEster

Di-n-hexylphthalate

$\mathscr{J}_{0} \quad$ BenzoicAcidMethylEster

EthylBenzoate

CarbonicAcidDimethylEster

CarbonicAcidDiethylEster

222.23

3.47750418

266.28869668

0

$0 \quad 2.7310869$

278.343

7.5314117

3.68549871

269.00881584

0 2.8210239

$$
334.4
$$

9.58331851

3.66527404

254.77418556

0

$\begin{array}{ll}0 & 2.8989693\end{array}$

136.148

3.8350845

150.175

3.97230232

3.57592247

294.8660173

$\begin{array}{ll}0 & 2.5302276\end{array}$

90.0779

3.19032943

3.69661406

296.49764136

$0 \quad 1.9995993$

118.131

4.17867589

3.26480345

257.35885798

0

0.89937

DiethyleneGlycolMonomethylEther

120.147

2.58790973

237.97766745

$0 \quad 1.1002293$

DiethyleneGlycolEthylEther

134.174

$3.54992886 \quad 3.80475544$

309.58597837

0.03

2322.249958

2.7490743

146.227

$2.81201639 \quad 4.4097601$

289.37938958

0.03

1844.421706

2.7790533

EthyleneGlycolMonohexylEther

DiethyleneGlycolMonobutylEther

162.227

$4.98877552 \quad 3.64722193$

329.31286074

0.03

1821.781926

2.2604166

AceticAnhydride

102.089

$3.83220077 \quad 3.22150598$

263.44573266

0.03

1706.006709

2.3503536

N,N-Diethylethanolamine

117.189

$2.5552308 \quad 4.20861965$

257.78962735

0

$0 \quad 2.788047$

332.34140138

0.03

1136.09683

2.4312969 
Table - continued from previous page

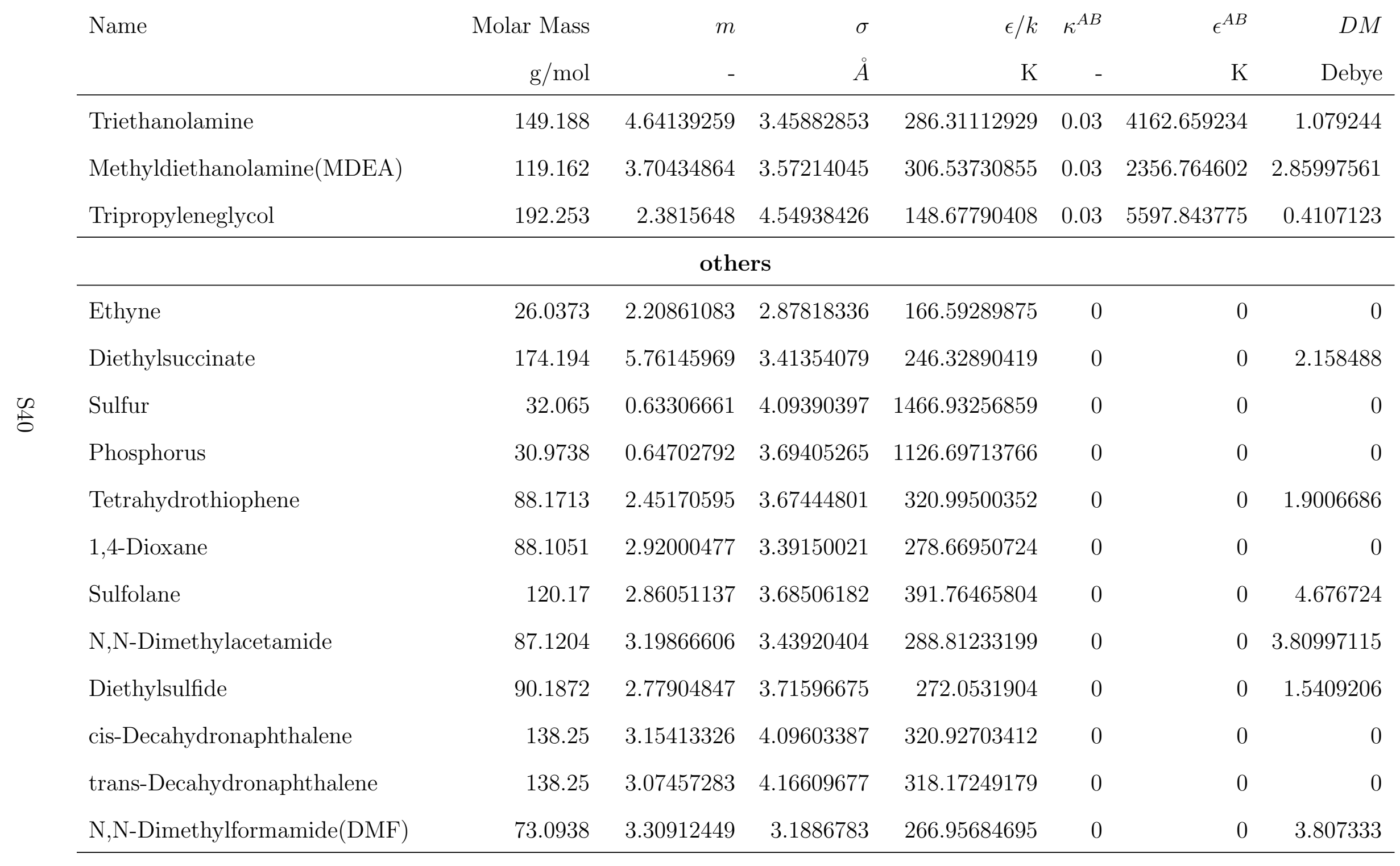


Table - continued from previous page

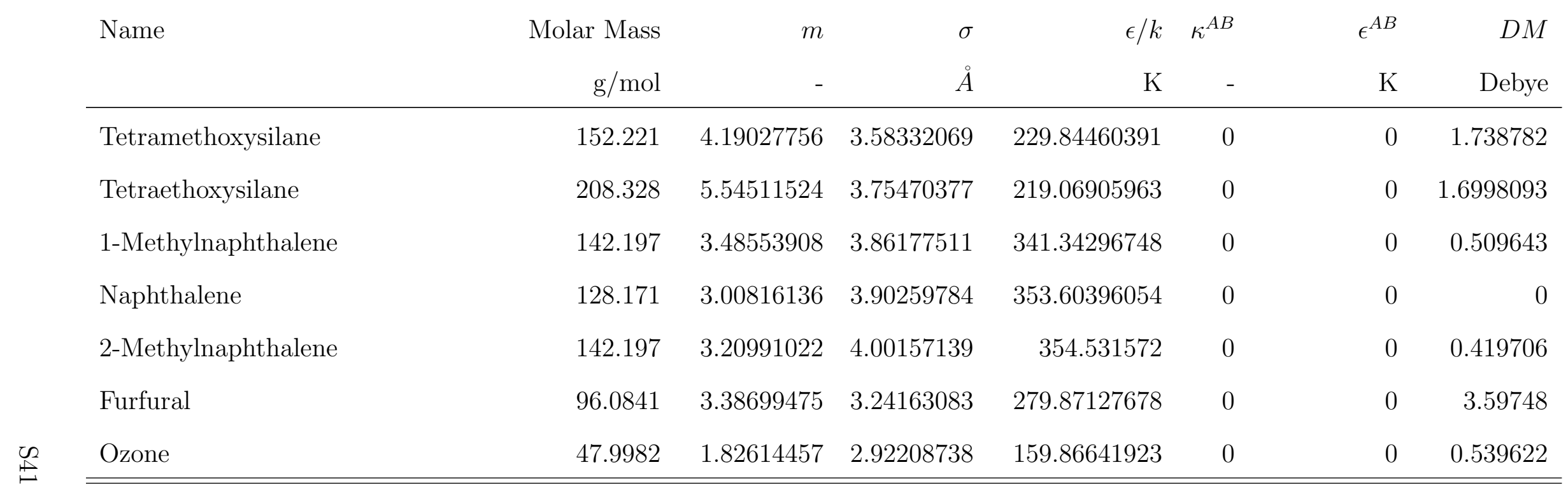

The quadropol moment is zero for all listed substances. 


\section{Group Decomposition}

Substances, which are represented as single segment:

- Methane: $\mathrm{CH}_{4}$

- Ethane: $\mathrm{H}_{3} \mathrm{C}-\mathrm{CH}_{3}$

- Methanol: $\mathrm{CH}_{3} \mathrm{OH}$

- Ethyne: $\mathrm{HC} \equiv \mathrm{CH}$

- Water: $\mathrm{H}_{2} \mathrm{O}$

- Carbon Dioxide: $\mathrm{CO}_{2}$

Group compositions for all other considered substances are given in the following table. $n^{t}$ is the total number of groups. To fit all columns on one page, we use the internal identifier "SI-Symbols" here, which is defined in table S4, in section Averaged Absolute Deviations. 
Table S7:

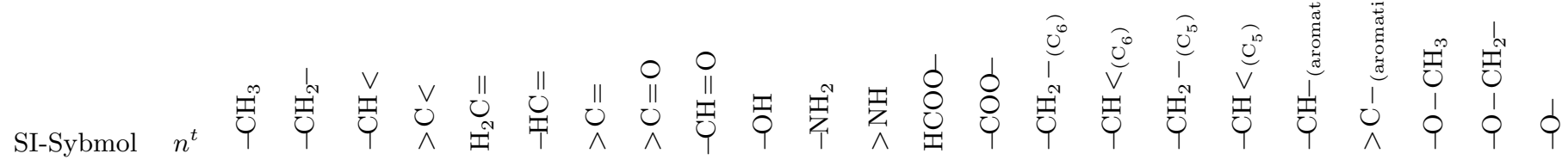

\section{Alkanes}

\begin{tabular}{|c|c|c|c|c|c|c|c|c|c|c|c|c|c|c|c|c|c|c|c|c|c|c|c|c|}
\hline A-3 & 3 & 2 & 1 & 0 & 0 & 0 & 0 & 0 & 0 & 0 & 0 & 0 & 0 & 0 & 0 & 0 & 0 & 0 & 0 & 0 & 0 & 0 & 0 & 0 \\
\hline A-4 & 4 & 2 & 2 & 0 & 0 & 0 & 0 & 0 & 0 & 0 & 0 & 0 & 0 & 0 & 0 & 0 & 0 & 0 & 0 & 0 & 0 & 0 & 0 & 0 \\
\hline A-5 & 5 & 2 & 3 & 0 & 0 & 0 & 0 & 0 & 0 & 0 & 0 & 0 & 0 & 0 & 0 & 0 & 0 & 0 & 0 & 0 & 0 & 0 & 0 & 0 \\
\hline A-6 & 6 & 2 & 4 & 0 & 0 & 0 & 0 & 0 & 0 & 0 & 0 & 0 & 0 & 0 & 0 & 0 & 0 & 0 & 0 & 0 & 0 & 0 & 0 & 0 \\
\hline A-7 & 7 & 2 & 5 & 0 & 0 & 0 & 0 & 0 & 0 & 0 & 0 & 0 & 0 & 0 & 0 & 0 & 0 & 0 & 0 & 0 & 0 & 0 & 0 & 0 \\
\hline A- 8 & 8 & 2 & 6 & 0 & 0 & 0 & 0 & 0 & 0 & 0 & 0 & 0 & 0 & 0 & 0 & 0 & 0 & 0 & 0 & 0 & 0 & 0 & 0 & 0 \\
\hline A-9 & 9 & 2 & 7 & 0 & 0 & 0 & 0 & 0 & 0 & 0 & 0 & 0 & 0 & 0 & 0 & 0 & 0 & 0 & 0 & 0 & 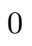 & 0 & 0 & 0 \\
\hline A-10 & 10 & 2 & 8 & 0 & 0 & 0 & 0 & 0 & 0 & 0 & 0 & 0 & 0 & 0 & 0 & 0 & 0 & 0 & 0 & 0 & 0 & 0 & 0 & 0 \\
\hline A-11 & 11 & 2 & 9 & 0 & 0 & 0 & 0 & 0 & 0 & 0 & 0 & 0 & 0 & 0 & 0 & 0 & 0 & 0 & 0 & 0 & 0 & 0 & 0 & 0 \\
\hline A-12 & 12 & 2 & 10 & 0 & 0 & 0 & 0 & 0 & 0 & 0 & 0 & 0 & 0 & 0 & 0 & 0 & 0 & 0 & 0 & 0 & U & 0 & 0 & 0 \\
\hline A-13 & 13 & 2 & 11 & 0 & 0 & 0 & 0 & 0 & 0 & 0 & 0 & 0 & 0 & 0 & 0 & 0 & 0 & 0 & 0 & 0 & 0 & 0 & 0 & 0 \\
\hline A-14 & 14 & 2 & 12 & 0 & 0 & 0 & 0 & 0 & 0 & 0 & 0 & 0 & 0 & 0 & 0 & 0 & 0 & 0 & 0 & 0 & U & 0 & 0 & 0 \\
\hline A-15 & 15 & 2 & 13 & 0 & 0 & 0 & 0 & 0 & 0 & 0 & 0 & 0 & 0 & 0 & 0 & 0 & 0 & 0 & 0 & 0 & 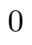 & 0 & 0 & 0 \\
\hline A-16 & 16 & 2 & 14 & 0 & 0 & 0 & 0 & 0 & 0 & 0 & 0 & 0 & 0 & 0 & 0 & 0 & 0 & 0 & 0 & 0 & & 0 & 0 & 0 \\
\hline A-17 & 17 & 2 & 15 & 0 & 0 & 0 & 0 & 0 & 0 & 0 & 0 & 0 & 0 & 0 & 0 & 0 & 0 & 0 & 0 & 0 & 0 & 0 & 0 & 0 \\
\hline A-18 & 18 & 2 & 16 & 0 & 0 & 0 & 0 & 0 & 0 & 0 & 0 & 0 & 0 & 0 & 0 & 0 & 0 & 0 & 0 & 0 & U & 0 & 0 & 0 \\
\hline A-19 & 19 & 2 & 17 & 0 & 0 & 0 & 0 & 0 & 0 & 0 & 0 & 0 & 0 & 0 & 0 & 0 & 0 & 0 & 0 & 0 & 0 & 0 & 0 & 0 \\
\hline A-20 & 20 & 2 & 18 & 0 & 0 & 0 & 0 & 0 & 0 & 0 & 0 & 0 & 0 & 0 & 0 & 0 & 0 & 0 & 0 & 0 & 0 & 0 & 0 & 0 \\
\hline A-21 & 21 & 2 & 19 & 0 & 0 & 0 & 0 & 0 & 0 & 0 & 0 & 0 & 0 & 0 & 0 & 0 & 0 & 0 & 0 & 0 & U & 0 & 0 & 0 \\
\hline A-22 & 22 & 2 & 20 & 0 & 0 & 0 & 0 & 0 & 0 & 0 & 0 & 0 & 0 & 0 & 0 & 0 & 0 & 0 & 0 & 0 & 0 & 0 & 0 & 0 \\
\hline A-23 & 23 & 2 & 21 & 0 & 0 & 0 & 0 & 0 & 0 & 0 & 0 & 0 & 0 & 0 & 0 & 0 & 0 & 0 & 0 & 0 & 0 & 0 & 0 & 0 \\
\hline A-24 & 24 & 2 & 22 & 0 & 0 & 0 & 0 & 0 & 0 & 0 & 0 & 0 & 0 & 0 & 0 & 0 & 0 & 0 & 0 & 0 & 0 & 0 & 0 & 0 \\
\hline
\end{tabular}

\section{Branched Alkanes}

\begin{tabular}{|c|c|c|c|c|c|c|c|c|c|c|c|c|c|c|c|c|c|c|c|c|c|c|c|c|}
\hline B-1 & 4 & 3 & 0 & 1 & 0 & 0 & 0 & 0 & 0 & 0 & 0 & 0 & 0 & 0 & 0 & 0 & 0 & 0 & 0 & 0 & 0 & 0 & 0 & 0 \\
\hline B-2 & 5 & 3 & 1 & 1 & 0 & 0 & 0 & 0 & 0 & 0 & 0 & 0 & 0 & 0 & 0 & 0 & 0 & 0 & 0 & 0 & 0 & 0 & 0 & 0 \\
\hline B-3 & 6 & 3 & 2 & 1 & 0 & 0 & 0 & 0 & 0 & 0 & 0 & 0 & 0 & 0 & 0 & 0 & 0 & 0 & 0 & 0 & 0 & 0 & 0 & 0 \\
\hline B-4 & 6 & 4 & 1 & 0 & 1 & 0 & 0 & 0 & 0 & 0 & 0 & 0 & 0 & 0 & 0 & 0 & 0 & 0 & 0 & 0 & 0 & 0 & 0 & 0 \\
\hline B-5 & 6 & 4 & 0 & 2 & 0 & 0 & 0 & 0 & 0 & 0 & 0 & 0 & 0 & 0 & 0 & 0 & 0 & 0 & 0 & 0 & 0 & 0 & 0 & 0 \\
\hline B-6 & 6 & 3 & 2 & 1 & 0 & 0 & 0 & 0 & 0 & 0 & 0 & 0 & 0 & 0 & 0 & 0 & 0 & 0 & 0 & 0 & 0 & 0 & 0 & 0 \\
\hline
\end{tabular}


Table - continued from previous page

\begin{tabular}{|c|c|c|c|c|c|c|c|c|c|c|c|c|c|c|c|c|c|c|c|c|c|c|c|c|}
\hline SI-Sybmol & $n^{t}$ & $\stackrel{\infty}{\dot{Y}}$ & $\underset{\mathrm{O}}{\stackrel{\mathrm{O}}{\mathrm{O}}}$ & $\begin{array}{l}V \\
\stackrel{\Xi}{U}\end{array}$ & $\begin{array}{l}\vee \\
\circlearrowright \\
\wedge\end{array}$ & $\bigcup_{N}^{\|}$ & 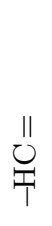 & $\begin{array}{l}\| \\
\circlearrowright \\
\wedge\end{array}$ & $\begin{array}{l}0 \\
\| \\
\circlearrowright \\
\wedge\end{array}$ & 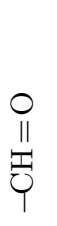 & $\stackrel{\square}{0}$ & 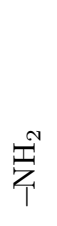 & $\underset{\wedge}{\stackrel{\text { 穵 }}{\wedge}}$ & 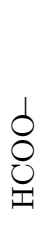 & 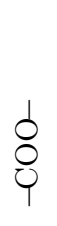 & $\overbrace{}^{0}$ & $\begin{array}{l}\overbrace{}^{0} \\
\vec{V} \\
\vec{U}\end{array}$ & 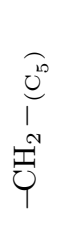 & 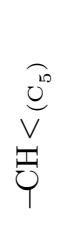 & 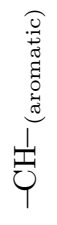 & 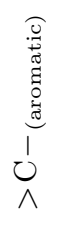 & $\begin{array}{c}\mathscr{I}_{0}^{\infty} \\
0 \\
1\end{array}$ & 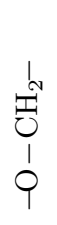 & 0 \\
\hline B-7 & 7 & 4 & 1 & 2 & 0 & 0 & 0 & 0 & 0 & 0 & 0 & 0 & 0 & 0 & 0 & 0 & 0 & 0 & 0 & 0 & 0 & 0 & 0 & 0 \\
\hline B-8 & 7 & 5 & 0 & 1 & 1 & 0 & 0 & 0 & 0 & 0 & 0 & 0 & 0 & 0 & 0 & 0 & 0 & 0 & 0 & 0 & 0 & 0 & 0 & 0 \\
\hline B-9 & 7 & 4 & 2 & 0 & 1 & 0 & 0 & 0 & 0 & 0 & 0 & 0 & 0 & 0 & 0 & 0 & 0 & 0 & 0 & 0 & 0 & 0 & 0 & 0 \\
\hline B-10 & 7 & 4 & 1 & 2 & 0 & 0 & 0 & 0 & 0 & 0 & 0 & 0 & 0 & 0 & 0 & 0 & 0 & 0 & 0 & 0 & 0 & 0 & 0 & 0 \\
\hline B-11 & 7 & 3 & 3 & 1 & 0 & 0 & 0 & 0 & 0 & 0 & 0 & 0 & 0 & 0 & 0 & 0 & 0 & 0 & 0 & 0 & 0 & 0 & 0 & 0 \\
\hline B-12 & 7 & 4 & 2 & 0 & 1 & 0 & 0 & 0 & 0 & 0 & 0 & 0 & 0 & 0 & 0 & 0 & 0 & 0 & 0 & 0 & 0 & 0 & 0 & 0 \\
\hline B-13 & 7 & 3 & 3 & 1 & 0 & 0 & 0 & 0 & 0 & 0 & 0 & 0 & 0 & 0 & 0 & 0 & 0 & 0 & 0 & 0 & 0 & 0 & 0 & 0 \\
\hline B-14 & 7 & 3 & 3 & 1 & 0 & 0 & 0 & 0 & 0 & 0 & 0 & 0 & 0 & 0 & 0 & 0 & 0 & 0 & 0 & 0 & 0 & 0 & 0 & 0 \\
\hline B-15 & 8 & 5 & 1 & 1 & 1 & 0 & 0 & 0 & 0 & 0 & 0 & 0 & 0 & 0 & 0 & 0 & 0 & 0 & 0 & 0 & 0 & 0 & 0 & 0 \\
\hline B-16 & 8 & 5 & 0 & 3 & 0 & 0 & 0 & 0 & 0 & 0 & 0 & 0 & 0 & 0 & 0 & 0 & 0 & 0 & 0 & 0 & 0 & 0 & 0 & 0 \\
\hline B-17 & 8 & 3 & 4 & 1 & 0 & 0 & 0 & 0 & 0 & 0 & 0 & 0 & 0 & 0 & 0 & 0 & 0 & 0 & 0 & 0 & 0 & 0 & 0 & 0 \\
\hline B-18 & 8 & 3 & 4 & 1 & 0 & 0 & 0 & 0 & 0 & 0 & 0 & 0 & 0 & 0 & 0 & 0 & 0 & 0 & 0 & 0 & 0 & 0 & 0 & 0 \\
\hline
\end{tabular}

\section{Alkene}

\begin{tabular}{|c|c|c|c|c|c|c|c|c|c|c|c|c|c|c|c|c|c|c|c|c|c|c|c|c|}
\hline C-1 & 2 & 0 & 0 & 0 & 0 & 2 & 0 & 0 & 0 & 0 & 0 & 0 & 0 & 0 & 0 & 0 & 0 & 0 & 0 & 0 & 0 & 0 & 0 & 0 \\
\hline C-2 & 3 & 1 & 0 & 0 & 0 & 1 & 1 & 0 & 0 & 0 & 0 & 0 & 0 & 0 & 0 & 0 & 0 & 0 & 0 & 0 & 0 & 0 & 0 & 0 \\
\hline C-3 & 4 & 1 & 1 & 0 & 0 & 1 & 1 & 0 & 0 & 0 & 0 & 0 & 0 & 0 & 0 & 0 & 0 & 0 & 0 & 0 & 0 & 0 & 0 & 0 \\
\hline C-4 & 4 & 2 & 0 & 0 & 0 & 1 & 0 & 1 & 0 & 0 & 0 & 0 & 0 & 0 & 0 & 0 & 0 & 0 & 0 & 0 & 0 & 0 & 0 & 0 \\
\hline C-5 & 4 & 2 & 0 & 0 & 0 & 0 & 2 & 0 & 0 & 0 & 0 & 0 & 0 & 0 & 0 & 0 & 0 & 0 & 0 & 0 & 0 & 0 & 0 & 0 \\
\hline C-6 & 4 & 2 & 0 & 0 & 0 & 0 & 2 & 0 & 0 & 0 & 0 & 0 & 0 & 0 & 0 & 0 & 0 & 0 & 0 & 0 & 0 & 0 & 0 & 0 \\
\hline C-7 & 5 & 1 & 0 & 0 & 0 & 2 & 1 & 1 & 0 & 0 & 0 & 0 & 0 & 0 & 0 & 0 & 0 & 0 & 0 & 0 & 0 & 0 & 0 & 0 \\
\hline C-8 & 5 & 1 & 2 & 0 & 0 & 1 & 1 & 0 & 0 & 0 & 0 & 0 & 0 & 0 & 0 & 0 & 0 & 0 & 0 & 0 & 0 & 0 & 0 & 0 \\
\hline C-9 & 6 & 3 & 0 & 1 & 0 & 1 & 0 & 1 & 0 & 0 & 0 & 0 & 0 & 0 & 0 & 0 & 0 & 0 & 0 & 0 & 0 & 0 & 0 & 0 \\
\hline C-10 & 6 & 4 & 0 & 0 & 0 & 0 & 0 & 2 & 0 & 0 & 0 & 0 & 0 & 0 & 0 & 0 & 0 & 0 & 0 & 0 & 0 & 0 & 0 & 0 \\
\hline C-11 & 6 & 1 & 3 & 0 & 0 & 1 & 1 & 0 & 0 & 0 & 0 & 0 & 0 & 0 & 0 & 0 & 0 & 0 & 0 & 0 & 0 & 0 & 0 & 0 \\
\hline C-12 & 7 & 1 & 4 & 0 & 0 & 1 & 1 & 0 & 0 & 0 & 0 & 0 & 0 & 0 & 0 & 0 & 0 & 0 & 0 & 0 & 0 & 0 & 0 & 0 \\
\hline C-13 & 7 & 2 & 3 & 0 & 0 & 0 & 2 & 0 & 0 & 0 & 0 & 0 & 0 & 0 & 0 & 0 & 0 & 0 & 0 & 0 & 0 & 0 & 0 & 0 \\
\hline C-14 & 8 & 1 & 5 & 0 & 0 & 1 & 1 & 0 & 0 & 0 & 0 & 0 & 0 & 0 & 0 & 0 & 0 & 0 & 0 & 0 & 0 & 0 & 0 & 0 \\
\hline C-15 & 9 & 1 & 6 & 0 & 0 & 1 & 1 & 0 & 0 & 0 & 0 & 0 & 0 & 0 & 0 & 0 & 0 & 0 & 0 & 0 & 0 & 0 & 0 & 0 \\
\hline C-16 & 10 & 1 & 7 & 0 & 0 & 1 & 1 & 0 & 0 & 0 & 0 & 0 & 0 & 0 & 0 & 0 & 0 & 0 & 0 & 0 & 0 & 0 & 0 & 0 \\
\hline C-17 & 11 & 1 & 8 & 0 & 0 & 1 & 1 & 0 & 0 & 0 & 0 & 0 & 0 & 0 & 0 & 0 & 0 & 0 & 0 & 0 & 0 & 0 & 0 & 0 \\
\hline C-18 & 12 & 1 & 9 & 0 & 0 & 1 & 1 & 0 & 0 & 0 & 0 & 0 & 0 & 0 & 0 & 0 & 0 & 0 & 0 & 0 & 0 & 0 & 0 & 0 \\
\hline
\end{tabular}


Table - continued from previous page

\begin{tabular}{|c|c|c|c|c|c|c|c|c|c|c|c|c|c|c|c|c|c|c|c|c|c|c|c|c|}
\hline SI-Sybmol & $n^{t}$ & 菅 & 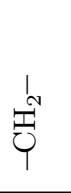 & $\begin{array}{l}V \\
\stackrel{D}{U}\end{array}$ & $\begin{array}{l}\vee \\
\cup \\
\wedge\end{array}$ & $\begin{array}{l}\| \\
U_{\Im} \\
\Xi^{\prime}\end{array}$ & $\underbrace{\|}_{0}$ & $\begin{array}{l}\| \\
\circlearrowright \\
\wedge\end{array}$ & $\begin{array}{l}0 \\
\| \\
0 \\
\wedge\end{array}$ & 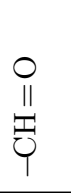 & 范 & $\mathbb{Z}_{i}^{N}$ & $\underset{\wedge}{\stackrel{M}{Z}}$ & $\begin{array}{l}0 \\
\bigcirc \\
0 \\
0\end{array}$ & $\begin{array}{l}\text { d } \\
\bigotimes \\
\bigotimes\end{array}$ & 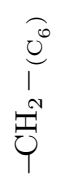 & 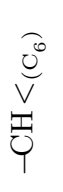 & 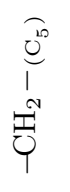 & 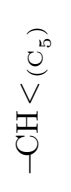 & 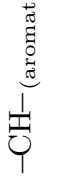 & 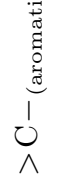 & $\begin{array}{l}\Xi_{0}^{\infty} \\
1 \\
0\end{array}$ & $\begin{array}{l}\text { I } \\
\stackrel{N}{0} \\
1 \\
0 \\
1\end{array}$ & d \\
\hline C-19 & 13 & 1 & 10 & 0 & 0 & 1 & 1 & 0 & 0 & 0 & 0 & 0 & 0 & 0 & 0 & 0 & 0 & 0 & 0 & 0 & 0 & 0 & 0 & 0 \\
\hline $\mathrm{C}-20$ & 14 & 1 & 11 & 0 & 0 & 1 & 1 & 0 & 0 & 0 & 0 & 0 & 0 & 0 & 0 & 0 & 0 & 0 & 0 & 0 & 0 & 0 & 0 & 0 \\
\hline $\mathrm{C}-21$ & 15 & 1 & 12 & 0 & 0 & 1 & 1 & 0 & 0 & 0 & 0 & 0 & 0 & 0 & 0 & 0 & 0 & 0 & 0 & 0 & 0 & 0 & 0 & 0 \\
\hline C-22 & 16 & 1 & 13 & 0 & 0 & 1 & 1 & 0 & 0 & 0 & 0 & 0 & 0 & 0 & 0 & 0 & 0 & 0 & 0 & 0 & 0 & 0 & 0 & 0 \\
\hline
\end{tabular}

Cyclic Alkanes

\begin{tabular}{|c|c|c|c|c|c|c|c|c|c|c|c|c|c|c|c|c|c|c|c|c|c|c|c|c|}
\hline D-1 & 5 & 0 & 0 & 0 & 0 & 0 & 0 & 0 & 0 & 0 & 0 & 0 & 0 & 0 & 0 & 0 & 0 & 5 & 0 & 0 & 0 & 0 & 0 & 0 \\
\hline D-2 & 6 & 0 & 0 & 0 & 0 & 0 & 0 & 0 & 0 & 0 & 0 & 0 & 0 & 0 & 0 & 6 & 0 & 0 & 0 & 0 & 0 & 0 & 0 & 0 \\
\hline D-3 & 7 & 1 & 0 & 0 & 0 & 0 & 0 & 0 & 0 & 0 & 0 & 0 & 0 & 0 & 0 & 5 & 1 & 0 & 0 & 0 & 0 & 0 & 0 & 0 \\
\hline D-4 & 8 & 1 & 1 & 0 & 0 & 0 & 0 & 0 & 0 & 0 & 0 & 0 & 0 & 0 & 0 & 5 & 1 & 0 & 0 & 0 & 0 & 0 & 0 & 0 \\
\hline D-5 & 8 & 2 & 0 & 0 & 0 & 0 & 0 & 0 & 0 & 0 & 0 & 0 & 0 & 0 & 0 & 4 & 2 & 0 & 0 & 0 & 0 & 0 & 0 & 0 \\
\hline D-6 & 8 & 2 & 0 & 0 & 0 & 0 & 0 & 0 & 0 & 0 & 0 & 0 & 0 & 0 & 0 & 4 & 2 & 0 & 0 & 0 & 0 & 0 & 0 & 0 \\
\hline D-7 & 8 & 2 & 0 & 0 & 0 & 0 & 0 & 0 & 0 & 0 & 0 & 0 & 0 & 0 & 0 & 4 & 2 & 0 & 0 & 0 & 0 & 0 & 0 & 0 \\
\hline D-8 & 8 & 2 & 0 & 0 & 0 & 0 & 0 & 0 & 0 & 0 & 0 & 0 & 0 & 0 & 0 & 4 & 2 & 0 & 0 & 0 & 0 & 0 & 0 & 0 \\
\hline D-9 & 9 & 1 & 2 & 0 & 0 & 0 & 0 & 0 & 0 & 0 & 0 & 0 & 0 & 0 & 0 & 5 & 1 & 0 & 0 & 0 & 0 & 0 & 0 & 0 \\
\hline D-10 & 6 & 1 & 0 & 0 & 0 & 0 & 0 & 0 & 0 & 0 & 0 & 0 & 0 & 0 & 0 & 0 & 0 & 4 & 1 & 0 & 0 & 0 & 0 & 0 \\
\hline D-11 & 8 & 2 & 0 & 0 & 0 & 0 & 0 & 0 & 0 & 0 & 0 & 0 & 0 & 0 & 0 & 4 & 2 & 0 & 0 & 0 & 0 & 0 & 0 & 0 \\
\hline
\end{tabular}

Aldehydes

\begin{tabular}{|c|c|c|c|c|c|c|c|c|c|c|c|c|c|c|c|c|c|c|c|c|c|c|c|c|}
\hline E-1 & 4 & 1 & 2 & 0 & 0 & 0 & 0 & 0 & 0 & 1 & 0 & 0 & 0 & 0 & 0 & 0 & 0 & 0 & 0 & 0 & 0 & 0 & 0 & 0 \\
\hline E-2 & 4 & 2 & 0 & 1 & 0 & 0 & 0 & 0 & 0 & 1 & 0 & 0 & 0 & 0 & 0 & 0 & 0 & 0 & 0 & 0 & 0 & 0 & 0 & 0 \\
\hline E-3 & 5 & 1 & 3 & 0 & 0 & 0 & 0 & 0 & 0 & 1 & 0 & 0 & 0 & 0 & 0 & 0 & 0 & 0 & 0 & 0 & 0 & 0 & 0 & 0 \\
\hline E-4 & 5 & 2 & 1 & 1 & 0 & 0 & 0 & 0 & 0 & 1 & 0 & 0 & 0 & 0 & 0 & 0 & 0 & 0 & 0 & 0 & 0 & 0 & 0 & 0 \\
\hline E-5 & 7 & 1 & 5 & 0 & 0 & 0 & 0 & 0 & 0 & 1 & 0 & 0 & 0 & 0 & 0 & 0 & 0 & 0 & 0 & 0 & 0 & 0 & 0 & 0 \\
\hline E-6 & 10 & 1 & 8 & 0 & 0 & 0 & 0 & 0 & 0 & 1 & 0 & 0 & 0 & 0 & 0 & 0 & 0 & 0 & 0 & 0 & 0 & 0 & 0 & 0 \\
\hline E-7 & 12 & 1 & 10 & 0 & 0 & 0 & 0 & 0 & 0 & 1 & 0 & 0 & 0 & 0 & 0 & 0 & 0 & 0 & 0 & 0 & 0 & 0 & 0 & 0 \\
\hline \multicolumn{25}{|c|}{ Aromatics } \\
\hline F-1 & 6 & 0 & 0 & 0 & 0 & 0 & 0 & 0 & 0 & 0 & 0 & 0 & 0 & 0 & 0 & 0 & 0 & 0 & 0 & 6 & 0 & 0 & 0 & 0 \\
\hline $\mathrm{F}-2$ & 7 & 1 & 0 & 0 & 0 & 0 & 0 & 0 & 0 & 0 & 0 & 0 & 0 & 0 & 0 & 0 & 0 & 0 & 0 & 5 & 1 & 0 & 0 & 0 \\
\hline F-3 & 8 & 1 & 1 & 0 & 0 & 0 & 0 & 0 & 0 & 0 & 0 & 0 & 0 & 0 & 0 & 0 & 0 & 0 & 0 & 5 & 1 & 0 & 0 & 0 \\
\hline F-4 & 8 & 2 & 0 & 0 & 0 & 0 & 0 & 0 & 0 & 0 & 0 & 0 & 0 & 0 & 0 & 0 & 0 & 0 & 0 & 4 & 2 & 0 & 0 & 0 \\
\hline F-5 & 8 & 2 & 0 & 0 & 0 & 0 & 0 & 0 & 0 & 0 & 0 & 0 & 0 & 0 & 0 & 0 & 0 & 0 & 0 & 4 & 2 & 0 & 0 & 0 \\
\hline F-6 & 8 & 2 & 0 & 0 & 0 & 0 & 0 & 0 & 0 & 0 & 0 & 0 & 0 & 0 & 0 & 0 & 0 & 0 & 0 & 4 & 2 & 0 & 0 & 0 \\
\hline
\end{tabular}


Table - continued from previous page

\begin{tabular}{|c|c|c|c|c|c|c|c|c|c|c|c|c|c|c|c|c|c|c|c|c|c|c|c|c|}
\hline SI-Sybmol & $n^{t}$ & $\stackrel{\infty}{0}$ & 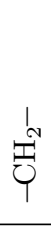 & $\begin{array}{l}V \\
\stackrel{V}{U}\end{array}$ & $\begin{array}{l}V \\
U \\
\wedge\end{array}$ & $\begin{array}{l}\| \\
\underset{N}{U} \\
\underset{J}{U}\end{array}$ & $\underset{⿱ 乛}{\bigcup_{T}^{\prime \prime}}$ & $\begin{array}{l}\| \\
\circlearrowright \\
\wedge\end{array}$ & $\begin{array}{l}0 \\
\| \\
0 \\
\wedge\end{array}$ & 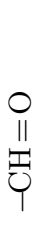 & $\stackrel{⿴ 囗 十)}{9}$ & $\stackrel{\mathbb{I}}{\mathrm{I}}^{\mathrm{I}}$ & 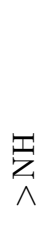 & $\begin{array}{l}0 \\
ٍ \\
ٍ \\
ٍ\end{array}$ & \begin{tabular}{l} 
ঠ \\
$ٍ$ \\
\hdashline
\end{tabular} & 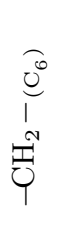 & $\begin{array}{l}\overbrace{}^{0} \\
\stackrel{V}{V} \\
\vec{U}\end{array}$ & 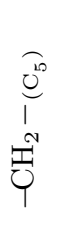 & 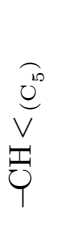 & 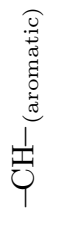 & 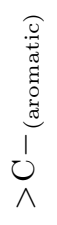 & $\begin{array}{c}\mathscr{I}^{\infty} \\
0 \\
0\end{array}$ & 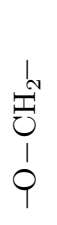 & 0 \\
\hline F-7 & 9 & 1 & 2 & 0 & 0 & 0 & 0 & 0 & 0 & 0 & 0 & 0 & 0 & 0 & 0 & 0 & 0 & 0 & 0 & 5 & 1 & 0 & 0 & 0 \\
\hline F-8 & 9 & 3 & 0 & 0 & 0 & 0 & 0 & 0 & 0 & 0 & 0 & 0 & 0 & 0 & 0 & 0 & 0 & 0 & 0 & 3 & 3 & 0 & 0 & 0 \\
\hline F-9 & 9 & 3 & 0 & 0 & 0 & 0 & 0 & 0 & 0 & 0 & 0 & 0 & 0 & 0 & 0 & 0 & 0 & 0 & 0 & 3 & 3 & 0 & 0 & 0 \\
\hline F-10 & 9 & 3 & 0 & 0 & 0 & 0 & 0 & 0 & 0 & 0 & 0 & 0 & 0 & 0 & 0 & 0 & 0 & 0 & 0 & 3 & 3 & 0 & 0 & 0 \\
\hline F-11 & 9 & 2 & 0 & 1 & 0 & 0 & 0 & 0 & 0 & 0 & 0 & 0 & 0 & 0 & 0 & 0 & 0 & 0 & 0 & 5 & 1 & 0 & 0 & 0 \\
\hline F-12 & 10 & 1 & 3 & 0 & 0 & 0 & 0 & 0 & 0 & 0 & 0 & 0 & 0 & 0 & 0 & 0 & 0 & 0 & 0 & 5 & 1 & 0 & 0 & 0 \\
\hline F-13 & 10 & 2 & 2 & 0 & 0 & 0 & 0 & 0 & 0 & 0 & 0 & 0 & 0 & 0 & 0 & 0 & 0 & 0 & 0 & 4 & 2 & 0 & 0 & 0 \\
\hline F-14 & 10 & 2 & 1 & 1 & 0 & 0 & 0 & 0 & 0 & 0 & 0 & 0 & 0 & 0 & 0 & 0 & 0 & 0 & 0 & 5 & 1 & 0 & 0 & 0 \\
\hline F-15 & 10 & 3 & 0 & 0 & 1 & 0 & 0 & 0 & 0 & 0 & 0 & 0 & 0 & 0 & 0 & 0 & 0 & 0 & 0 & 5 & 1 & 0 & 0 & 0 \\
\hline F-16 & 15 & 6 & 0 & 3 & 0 & 0 & 0 & 0 & 0 & 0 & 0 & 0 & 0 & 0 & 0 & 0 & 0 & 0 & 0 & 3 & 3 & 0 & 0 & 0 \\
\hline
\end{tabular}

\section{Ketones}

\begin{tabular}{|c|c|c|c|c|c|c|c|c|c|c|c|c|c|c|c|c|c|c|c|c|c|c|c|c|}
\hline G-1 & 3 & 2 & 0 & 0 & 0 & 0 & 0 & 0 & 1 & 0 & 0 & 0 & 0 & 0 & 0 & 0 & 0 & 0 & 0 & 0 & 0 & 0 & 0 & 0 \\
\hline G-2 & 4 & 2 & 1 & 0 & 0 & 0 & 0 & 0 & 1 & 0 & 0 & 0 & 0 & 0 & 0 & 0 & 0 & 0 & 0 & 0 & 0 & 0 & 0 & 0 \\
\hline G-3 & 5 & 2 & 2 & 0 & 0 & 0 & 0 & 0 & 1 & 0 & 0 & 0 & 0 & 0 & 0 & 0 & 0 & 0 & 0 & 0 & 0 & 0 & 0 & 0 \\
\hline G-4 & 5 & 2 & 2 & 0 & 0 & 0 & 0 & 0 & 1 & 0 & 0 & 0 & 0 & 0 & 0 & 0 & 0 & 0 & 0 & 0 & 0 & 0 & 0 & 0 \\
\hline G-5 & 6 & 3 & 1 & 1 & 0 & 0 & 0 & 0 & 1 & 0 & 0 & 0 & 0 & 0 & 0 & 0 & 0 & 0 & 0 & 0 & 0 & 0 & 0 & 0 \\
\hline G-6 & 6 & 2 & 3 & 0 & 0 & 0 & 0 & 0 & 1 & 0 & 0 & 0 & 0 & 0 & 0 & 0 & 0 & 0 & 0 & 0 & 0 & 0 & 0 & 0 \\
\hline G-7 & 6 & 2 & 3 & 0 & 0 & 0 & 0 & 0 & 1 & 0 & 0 & 0 & 0 & 0 & 0 & 0 & 0 & 0 & 0 & 0 & 0 & 0 & 0 & 0 \\
\hline G-8 & 7 & 2 & 4 & 0 & 0 & 0 & 0 & 0 & 1 & 0 & 0 & 0 & 0 & 0 & 0 & 0 & 0 & 0 & 0 & 0 & 0 & 0 & 0 & 0 \\
\hline G-9 & 7 & 2 & 4 & 0 & 0 & 0 & 0 & 0 & 1 & 0 & 0 & 0 & 0 & 0 & 0 & 0 & 0 & 0 & 0 & 0 & 0 & 0 & 0 & 0 \\
\hline G-10 & 7 & 2 & 4 & 0 & 0 & 0 & 0 & 0 & 1 & 0 & 0 & 0 & 0 & 0 & 0 & 0 & 0 & 0 & 0 & 0 & 0 & 0 & 0 & 0 \\
\hline G-11 & 8 & 2 & 5 & 0 & 0 & 0 & 0 & 0 & 1 & 0 & 0 & 0 & 0 & 0 & 0 & 0 & 0 & 0 & 0 & 0 & 0 & 0 & 0 & 0 \\
\hline G-12 & 9 & 2 & 6 & 0 & 0 & 0 & 0 & 0 & 1 & 0 & 0 & 0 & 0 & 0 & 0 & 0 & 0 & 0 & 0 & 0 & 0 & 0 & 0 & 0 \\
\hline G-13 & 11 & 2 & 8 & 0 & 0 & 0 & 0 & 0 & 1 & 0 & 0 & 0 & 0 & 0 & 0 & 0 & 0 & 0 & 0 & 0 & 0 & 0 & 0 & 0 \\
\hline G-14 & 10 & 2 & 7 & 0 & 0 & 0 & 0 & 0 & 1 & 0 & 0 & 0 & 0 & 0 & 0 & 0 & 0 & 0 & 0 & 0 & 0 & 0 & 0 & 0 \\
\hline G-15 & 11 & 2 & 8 & 0 & 0 & 0 & 0 & 0 & 1 & 0 & 0 & 0 & 0 & 0 & 0 & 0 & 0 & 0 & 0 & 0 & 0 & 0 & 0 & 0 \\
\hline G-16 & 12 & 2 & 9 & 0 & 0 & 0 & 0 & 0 & 1 & 0 & 0 & 0 & 0 & 0 & 0 & 0 & 0 & 0 & 0 & 0 & 0 & 0 & 0 & 0 \\
\hline G-17 & 13 & 2 & 10 & 0 & 0 & 0 & 0 & 0 & 1 & 0 & 0 & 0 & 0 & 0 & 0 & 0 & 0 & 0 & 0 & 0 & 0 & 0 & 0 & 0 \\
\hline G-18 & 13 & 4 & 6 & 2 & 0 & 0 & 0 & 0 & 1 & 0 & 0 & 0 & 0 & 0 & 0 & 0 & 0 & 0 & 0 & 0 & 0 & 0 & 0 & 0 \\
\hline \multicolumn{25}{|c|}{ Ester } \\
\hline $\mathrm{H}-1$ & 2 & 1 & 0 & 0 & 0 & 0 & 0 & 0 & 0 & 0 & 0 & 0 & 0 & 1 & 0 & 0 & 0 & 0 & 0 & 0 & 0 & 0 & 0 & 0 \\
\hline
\end{tabular}


Table - continued from previous page

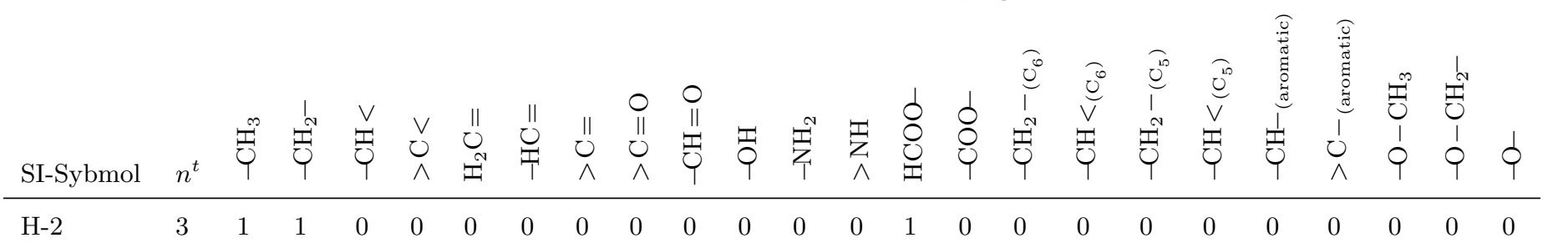

$\begin{array}{lllllllllllllllllllllllllll}\mathrm{H}-3 & 4 & 1 & 2 & 0 & 0 & 0 & 0 & 0 & 0 & 0 & 0 & 0 & 0 & 1 & 0 & 0 & 0 & 0 & 0 & 0 & 0 & 0 & 0 & 0\end{array}$

H-4 $\quad 5 \quad \begin{array}{llllllllllllllllllllllll} & 5 & 3 & 0 & 0 & 0 & 0 & 0 & 0 & 0 & 0 & 0 & 0 & 1 & 0 & 0 & 0 & 0 & 0 & 0 & 0 & 0 & 0 & 0\end{array}$

H-5 $\quad 6 \begin{array}{lllllllllllllllllllllllll} & 6 & 1 & 4 & 0 & 0 & 0 & 0 & 0 & 0 & 0 & 0 & 0 & 0 & 1 & 0 & 0 & 0 & 0 & 0 & 0 & 0 & 0 & 0 & 0\end{array}$

H-6 $\quad \begin{array}{llllllllllllllllllllllllll} & 8 & 1 & 6 & 0 & 0 & 0 & 0 & 0 & 0 & 0 & 0 & 0 & 0 & 1 & 0 & 0 & 0 & 0 & 0 & 0 & 0 & 0 & 0 & 0\end{array}$

$\begin{array}{llllllllllllllllllllllllll}\mathrm{H}-7 & 9 & 1 & 7 & 0 & 0 & 0 & 0 & 0 & 0 & 0 & 0 & 0 & 0 & 1 & 0 & 0 & 0 & 0 & 0 & 0 & 0 & 0 & 0 & 0\end{array}$

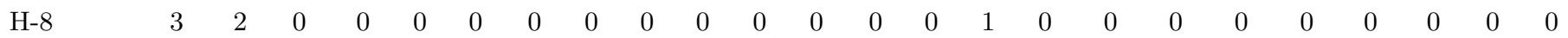

H-9 $\quad 4 \quad \begin{array}{lllllllllllllllllllllllll} & 4 & 1 & 0 & 0 & 0 & 0 & 0 & 0 & 0 & 0 & 0 & 0 & 0 & 1 & 0 & 0 & 0 & 0 & 0 & 0 & 0 & 0 & 0\end{array}$

H-10 $\quad 5 \quad \begin{array}{llllllllllllllllllllllll} & 5 & 0 & 1 & 0 & 0 & 0 & 0 & 0 & 0 & 0 & 0 & 0 & 0 & 1 & 0 & 0 & 0 & 0 & 0 & 0 & 0 & 0 & 0\end{array}$

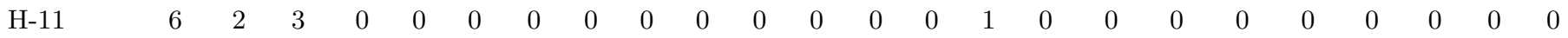

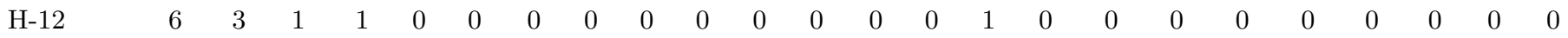

H-13 $\quad 7 \quad \begin{array}{lllllllllllllllllllllllll} & 7 & 2 & 4 & 0 & 0 & 0 & 0 & 0 & 0 & 0 & 0 & 0 & 0 & 0 & 1 & 0 & 0 & 0 & 0 & 0 & 0 & 0 & 0 & 0\end{array}$

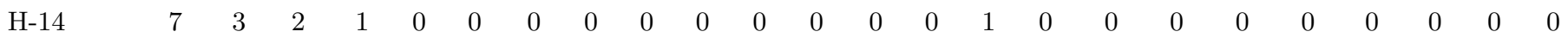

H-15 $\quad 8 \begin{array}{lllllllllllllllllllllllll} & 8 & 5 & 0 & 0 & 0 & 0 & 0 & 0 & 0 & 0 & 0 & 0 & 0 & 1 & 0 & 0 & 0 & 0 & 0 & 0 & 0 & 0 & 0\end{array}$

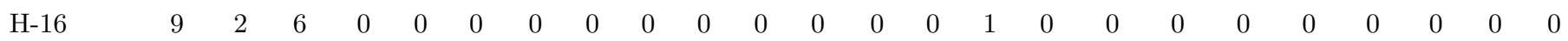

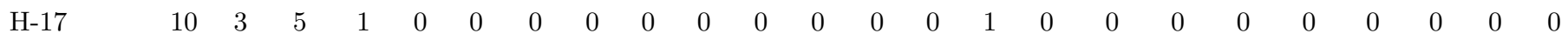

H-18 $\quad 6 \begin{array}{lllllllllllllllllllllllll} & 6 & 2 & 3 & 0 & 0 & 0 & 0 & 0 & 0 & 0 & 0 & 0 & 0 & 0 & 1 & 0 & 0 & 0 & 0 & 0 & 0 & 0 & 0 & 0\end{array}$

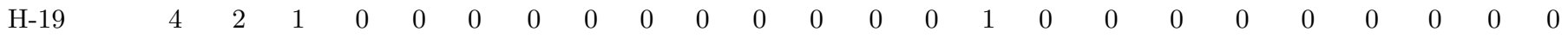

H-20 $\quad \begin{array}{lllllllllllllllllllllllll} & 7 & 2 & 4 & 0 & 0 & 0 & 0 & 0 & 0 & 0 & 0 & 0 & 0 & 0 & 1 & 0 & 0 & 0 & 0 & 0 & 0 & 0 & 0 & 0\end{array}$

$\begin{array}{llllllllllllllllllllllllllll}\text { H-21 } & 22 & 2 & 19 & 0 & 0 & 0 & 0 & 0 & 0 & 0 & 0 & 0 & 0 & 0 & 1 & 0 & 0 & 0 & 0 & 0 & 0 & 0 & 0 & 0\end{array}$

$\begin{array}{lllllllllllllllllllllllll}\mathrm{H}-22 & 4 & 2 & 0 & 1 & 0 & 0 & 0 & 0 & 0 & 0 & 0 & 0 & 0 & 1 & 0 & 0 & 0 & 0 & 0 & 0 & 0 & 0 & 0 & 0\end{array}$

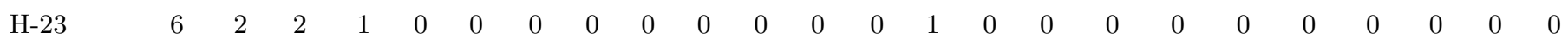

$\begin{array}{llllllllllllllllllllllllll}\mathrm{H}-24 & 7 & 1 & 5 & 0 & 0 & 0 & 0 & 0 & 0 & 0 & 0 & 0 & 0 & 1 & 0 & 0 & 0 & 0 & 0 & 0 & 0 & 0 & 0 & 0\end{array}$

$\begin{array}{lllllllllllllllllllllllll}\mathrm{H}-25 & 7 & 2 & 3 & 1 & 0 & 0 & 0 & 0 & 0 & 0 & 0 & 0 & 0 & 1 & 0 & 0 & 0 & 0 & 0 & 0 & 0 & 0 & 0 & 0\end{array}$

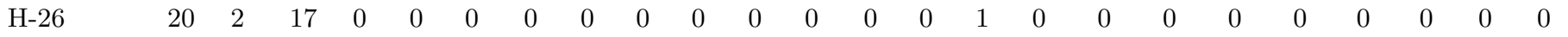

$\begin{array}{lllllllllllllllllllllllll}\mathrm{H}-27 & 7 & 3 & 2 & 1 & 0 & 0 & 0 & 0 & 0 & 0 & 0 & 0 & 0 & 0 & 1 & 0 & 0 & 0 & 0 & 0 & 0 & 0 & 0 & 0\end{array}$

H-28 $\quad 9 \begin{array}{lllllllllllllllllllllllll} & 9 & 6 & 0 & 0 & 0 & 0 & 0 & 0 & 0 & 0 & 0 & 0 & 0 & 1 & 0 & 0 & 0 & 0 & 0 & 0 & 0 & 0 & 0\end{array}$

$\begin{array}{lllllllllllllllllllllllll}\mathrm{H}-29 & 9 & 3 & 4 & 1 & 0 & 0 & 0 & 0 & 0 & 0 & 0 & 0 & 0 & 0 & 1 & 0 & 0 & 0 & 0 & 0 & 0 & 0 & 0 & 0\end{array}$

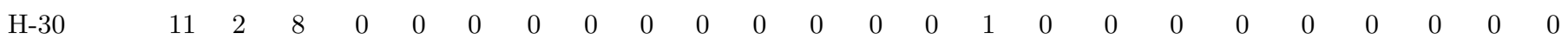

H-31 $\quad 8 \begin{array}{llllllllllllllllllllllll} & 8 & 5 & 0 & 0 & 0 & 0 & 0 & 0 & 0 & 0 & 0 & 0 & 0 & 1 & 0 & 0 & 0 & 0 & 0 & 0 & 0 & 0 & 0\end{array}$

\begin{tabular}{llllllllllllllllllllllllll}
$\mathrm{H}-32$ & 8 & 3 & 3 & 1 & 0 & 0 & 0 & 0 & 0 & 0 & 0 & 0 & 0 & 0 & 1 & 0 & 0 & 0 & 0 & 0 & 0 & 0 & 0 & 0 \\
\hline
\end{tabular}

Continued on next page 
Table - continued from previous page

\begin{tabular}{|c|c|c|c|c|c|c|c|c|c|c|c|c|c|c|c|c|c|c|c|c|c|c|c|c|}
\hline SI-Sybmol & $n^{t}$ & $\stackrel{\infty}{\mathcal{O}}$ & $\underset{⿱ 乛}{\stackrel{S}{S}}$ & $\stackrel{V}{\stackrel{V}{Z}}$ & $\begin{array}{l}\vee \\
\circlearrowright \\
\wedge\end{array}$ & $\begin{array}{l}\| \\
\bigcup_{N} \\
\mathcal{I}^{\prime}\end{array}$ & $\underset{⿱ ⺌}{\|}$ & $\begin{array}{l}\| \\
\circlearrowright\end{array}$ & $\begin{array}{l}0 \\
\| \\
\circlearrowright \\
\wedge\end{array}$ & 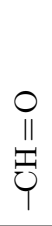 & $\stackrel{T}{0}$ & $\underset{\mathbf{Z}^{N}}{\mathbb{N}}$ & $\underset{\wedge}{\stackrel{\text { Z }}{\wedge}}$ & 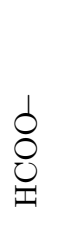 & $\underset{\wp}{ٍ}$ & 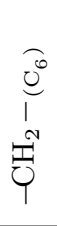 & 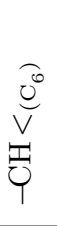 & 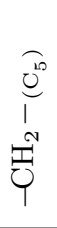 & 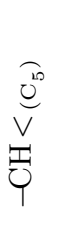 & 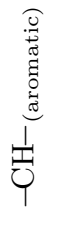 & 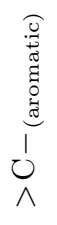 & $\begin{array}{c}\mathscr{I}_{1}^{\infty} \\
0\end{array}$ & 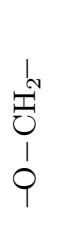 & 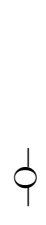 \\
\hline H-33 & 9 & 2 & 6 & 0 & 0 & 0 & 0 & 0 & 0 & 0 & 0 & 0 & 0 & 0 & 1 & 0 & 0 & 0 & 0 & 0 & 0 & 0 & 0 & 0 \\
\hline H-34 & 10 & 2 & 7 & 0 & 0 & 0 & 0 & 0 & 0 & 0 & 0 & 0 & 0 & 0 & 1 & 0 & 0 & 0 & 0 & 0 & 0 & 0 & 0 & 0 \\
\hline H-35 & 11 & 2 & 8 & 0 & 0 & 0 & 0 & 0 & 0 & 0 & 0 & 0 & 0 & 0 & 1 & 0 & 0 & 0 & 0 & 0 & 0 & 0 & 0 & 0 \\
\hline H-36 & 6 & 2 & 3 & 0 & 0 & 0 & 0 & 0 & 0 & 0 & 0 & 0 & 0 & 0 & 1 & 0 & 0 & 0 & 0 & 0 & 0 & 0 & 0 & 0 \\
\hline H-37 & 10 & 2 & 7 & 0 & 0 & 0 & 0 & 0 & 0 & 0 & 0 & 0 & 0 & 0 & 1 & 0 & 0 & 0 & 0 & 0 & 0 & 0 & 0 & 0 \\
\hline H-38 & 10 & 3 & 5 & 1 & 0 & 0 & 0 & 0 & 0 & 0 & 0 & 0 & 0 & 0 & 1 & 0 & 0 & 0 & 0 & 0 & 0 & 0 & 0 & 0 \\
\hline H-39 & 7 & 2 & 4 & 0 & 0 & 0 & 0 & 0 & 0 & 0 & 0 & 0 & 0 & 0 & 1 & 0 & 0 & 0 & 0 & 0 & 0 & 0 & 0 & 0 \\
\hline $\mathrm{H}-40$ & 8 & 2 & 5 & 0 & 0 & 0 & 0 & 0 & 0 & 0 & 0 & 0 & 0 & 0 & 1 & 0 & 0 & 0 & 0 & 0 & 0 & 0 & 0 & 0 \\
\hline H-41 & 9 & 2 & 6 & 0 & 0 & 0 & 0 & 0 & 0 & 0 & 0 & 0 & 0 & 0 & 1 & 0 & 0 & 0 & 0 & 0 & 0 & 0 & 0 & 0 \\
\hline H- 42 & 10 & 2 & 7 & 0 & 0 & 0 & 0 & 0 & 0 & 0 & 0 & 0 & 0 & 0 & 1 & 0 & 0 & 0 & 0 & 0 & 0 & 0 & 0 & 0 \\
\hline H-43 & 11 & 2 & 8 & 0 & 0 & 0 & 0 & 0 & 0 & 0 & 0 & 0 & 0 & 0 & 1 & 0 & 0 & 0 & 0 & 0 & 0 & 0 & 0 & 0 \\
\hline H-44 & 12 & 2 & 9 & 0 & 0 & 0 & 0 & 0 & 0 & 0 & 0 & 0 & 0 & 0 & 1 & 0 & 0 & 0 & 0 & 0 & 0 & 0 & 0 & 0 \\
\hline H-45 & 13 & 2 & 10 & 0 & 0 & 0 & 0 & 0 & 0 & 0 & 0 & 0 & 0 & 0 & 1 & 0 & 0 & 0 & 0 & 0 & 0 & 0 & 0 & 0 \\
\hline H-46 & 14 & 2 & 11 & 0 & 0 & 0 & 0 & 0 & 0 & 0 & 0 & 0 & 0 & 0 & 1 & 0 & 0 & 0 & 0 & 0 & 0 & 0 & 0 & 0 \\
\hline H-47 & 15 & 2 & 12 & 0 & 0 & 0 & 0 & 0 & 0 & 0 & 0 & 0 & 0 & 0 & 1 & 0 & 0 & 0 & 0 & 0 & 0 & 0 & 0 & 0 \\
\hline $\mathrm{H}-48$ & 9 & 2 & 6 & 0 & 0 & 0 & 0 & 0 & 0 & 0 & 0 & 0 & 0 & 0 & 1 & 0 & 0 & 0 & 0 & 0 & 0 & 0 & 0 & 0 \\
\hline H-49 & 20 & 2 & 17 & 0 & 0 & 0 & 0 & 0 & 0 & 0 & 0 & 0 & 0 & 0 & 1 & 0 & 0 & 0 & 0 & 0 & 0 & 0 & 0 & 0 \\
\hline H-50 & 19 & 2 & 16 & 0 & 0 & 0 & 0 & 0 & 0 & 0 & 0 & 0 & 0 & 0 & 1 & 0 & 0 & 0 & 0 & 0 & 0 & 0 & 0 & 0 \\
\hline
\end{tabular}

\section{Alcohols}

\begin{tabular}{|c|c|c|c|c|c|c|c|c|c|c|c|c|c|c|c|c|c|c|c|c|c|c|c|c|}
\hline K-2 & 3 & 1 & 1 & 0 & 0 & 0 & 0 & 0 & 0 & 0 & 1 & 0 & 0 & 0 & 0 & 0 & 0 & 0 & 0 & 0 & 0 & 0 & 0 & 0 \\
\hline K-3 & 4 & 1 & 2 & 0 & 0 & 0 & 0 & 0 & 0 & 0 & 1 & 0 & 0 & 0 & 0 & 0 & 0 & 0 & 0 & 0 & 0 & 0 & 0 & 0 \\
\hline K-4 & 4 & 2 & 0 & 1 & 0 & 0 & 0 & 0 & 0 & 0 & 1 & 0 & 0 & 0 & 0 & 0 & 0 & 0 & 0 & 0 & 0 & 0 & 0 & 0 \\
\hline K-5 & 5 & 1 & 3 & 0 & 0 & 0 & 0 & 0 & 0 & 0 & 1 & 0 & 0 & 0 & 0 & 0 & 0 & 0 & 0 & 0 & 0 & 0 & 0 & 0 \\
\hline K-6 & 5 & 2 & 1 & 1 & 0 & 0 & 0 & 0 & 0 & 0 & 1 & 0 & 0 & 0 & 0 & 0 & 0 & 0 & 0 & 0 & 0 & 0 & 0 & 0 \\
\hline K-7 & 6 & 1 & 4 & 0 & 0 & 0 & 0 & 0 & 0 & 0 & 1 & 0 & 0 & 0 & 0 & 0 & 0 & 0 & 0 & 0 & 0 & 0 & 0 & 0 \\
\hline K-8 & 6 & 2 & 2 & 1 & 0 & 0 & 0 & 0 & 0 & 0 & 1 & 0 & 0 & 0 & 0 & 0 & 0 & 0 & 0 & 0 & 0 & 0 & 0 & 0 \\
\hline K-9 & 7 & 1 & 5 & 0 & 0 & 0 & 0 & 0 & 0 & 0 & 1 & 0 & 0 & 0 & 0 & 0 & 0 & 0 & 0 & 0 & 0 & 0 & 0 & 0 \\
\hline K-10 & 8 & 1 & 6 & 0 & 0 & 0 & 0 & 0 & 0 & 0 & 1 & 0 & 0 & 0 & 0 & 0 & 0 & 0 & 0 & 0 & 0 & 0 & 0 & 0 \\
\hline $\mathrm{K}-11$ & 9 & 1 & 7 & 0 & 0 & 0 & 0 & 0 & 0 & 0 & 1 & 0 & 0 & 0 & 0 & 0 & 0 & 0 & 0 & 0 & 0 & 0 & 0 & 0 \\
\hline $\mathrm{K}-12$ & 9 & 2 & 5 & 1 & 0 & 0 & 0 & 0 & 0 & 0 & 1 & 0 & 0 & 0 & 0 & 0 & 0 & 0 & 0 & 0 & 0 & 0 & 0 & 0 \\
\hline K-13 & 10 & 1 & 8 & 0 & 0 & 0 & 0 & 0 & 0 & 0 & 1 & 0 & 0 & 0 & 0 & 0 & 0 & 0 & 0 & 0 & 0 & 0 & 0 & 0 \\
\hline
\end{tabular}


Table - continued from previous page

\begin{tabular}{|c|c|c|c|c|c|c|c|c|c|c|c|c|c|c|c|c|c|c|c|c|c|c|c|c|}
\hline SI-Sybmol & $n^{t}$ & $\stackrel{\infty}{\mathcal{O}}$ & 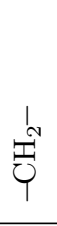 & $\stackrel{V}{\stackrel{V}{Z}}$ & $\begin{array}{l}\vee \\
\circlearrowright \\
\wedge\end{array}$ & $\begin{array}{l}\| \\
\bigcup_{N} \\
\mathcal{I}^{\prime}\end{array}$ & $\underset{⿱ ⺌}{\|}$ & $\begin{array}{l}\| \\
\circlearrowright\end{array}$ & $\begin{array}{l}0 \\
\| \\
\circlearrowright \\
\wedge\end{array}$ & $\begin{array}{c}\underset{\|}{\|} \\
\stackrel{\vec{O}}{O}\end{array}$ & $\stackrel{\square}{9}$ & $\underset{\mathbf{Z}^{N}}{\mathbb{N}}$ & $\underset{\wedge}{\stackrel{\text { Z }}{\wedge}}$ & 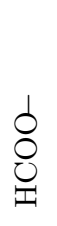 & $\underset{\wp}{ٍ}$ & 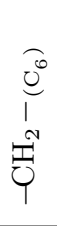 & 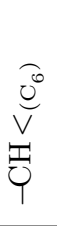 & 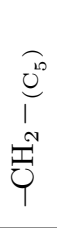 & 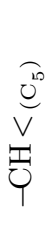 & 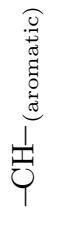 & 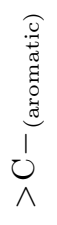 & $\begin{array}{c}\mathscr{I}_{1}^{\infty} \\
0\end{array}$ & 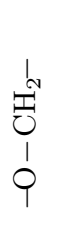 & 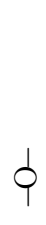 \\
\hline $\mathrm{K}-14$ & 11 & 1 & 9 & 0 & 0 & 0 & 0 & 0 & 0 & 0 & 1 & 0 & 0 & 0 & 0 & 0 & 0 & 0 & 0 & 0 & 0 & 0 & 0 & 0 \\
\hline $\mathrm{K}-15$ & 12 & 1 & 10 & 0 & 0 & 0 & 0 & 0 & 0 & 0 & 1 & 0 & 0 & 0 & 0 & 0 & 0 & 0 & 0 & 0 & 0 & 0 & 0 & 0 \\
\hline $\mathrm{K}-16$ & 13 & 1 & 11 & 0 & 0 & 0 & 0 & 0 & 0 & 0 & 1 & 0 & 0 & 0 & 0 & 0 & 0 & 0 & 0 & 0 & 0 & 0 & 0 & 0 \\
\hline $\mathrm{K}-17$ & 15 & 1 & 13 & 0 & 0 & 0 & 0 & 0 & 0 & 0 & 1 & 0 & 0 & 0 & 0 & 0 & 0 & 0 & 0 & 0 & 0 & 0 & 0 & 0 \\
\hline $\mathrm{K}-18$ & 17 & 1 & 15 & 0 & 0 & 0 & 0 & 0 & 0 & 0 & 1 & 0 & 0 & 0 & 0 & 0 & 0 & 0 & 0 & 0 & 0 & 0 & 0 & 0 \\
\hline K-19 & 19 & 1 & 17 & 0 & 0 & 0 & 0 & 0 & 0 & 0 & 1 & 0 & 0 & 0 & 0 & 0 & 0 & 0 & 0 & 0 & 0 & 0 & 0 & 0 \\
\hline $\mathrm{K}-20$ & 5 & 3 & 0 & 0 & 1 & 0 & 0 & 0 & 0 & 0 & 1 & 0 & 0 & 0 & 0 & 0 & 0 & 0 & 0 & 0 & 0 & 0 & 0 & 0 \\
\hline $\mathrm{K}-21$ & 5 & 2 & 1 & 1 & 0 & 0 & 0 & 0 & 0 & 0 & 1 & 0 & 0 & 0 & 0 & 0 & 0 & 0 & 0 & 0 & 0 & 0 & 0 & 0 \\
\hline $\mathrm{K}-22$ & 6 & 2 & 2 & 1 & 0 & 0 & 0 & 0 & 0 & 0 & 1 & 0 & 0 & 0 & 0 & 0 & 0 & 0 & 0 & 0 & 0 & 0 & 0 & 0 \\
\hline $\mathrm{K}-23$ & 6 & 2 & 2 & 1 & 0 & 0 & 0 & 0 & 0 & 0 & 1 & 0 & 0 & 0 & 0 & 0 & 0 & 0 & 0 & 0 & 0 & 0 & 0 & 0 \\
\hline $\mathrm{K}-24$ & 7 & 4 & 0 & 1 & 1 & 0 & 0 & 0 & 0 & 0 & 1 & 0 & 0 & 0 & 0 & 0 & 0 & 0 & 0 & 0 & 0 & 0 & 0 & 0 \\
\hline $\mathrm{K}-25$ & 7 & 3 & 2 & 0 & 1 & 0 & 0 & 0 & 0 & 0 & 1 & 0 & 0 & 0 & 0 & 0 & 0 & 0 & 0 & 0 & 0 & 0 & 0 & 0 \\
\hline $\mathrm{K}-26$ & 7 & 4 & 0 & 1 & 1 & 0 & 0 & 0 & 0 & 0 & 1 & 0 & 0 & 0 & 0 & 0 & 0 & 0 & 0 & 0 & 0 & 0 & 0 & 0 \\
\hline $\mathrm{K}-27$ & 8 & 3 & 3 & 0 & 1 & 0 & 0 & 0 & 0 & 0 & 1 & 0 & 0 & 0 & 0 & 0 & 0 & 0 & 0 & 0 & 0 & 0 & 0 & 0 \\
\hline $\mathrm{K}-28$ & 8 & 2 & 4 & 1 & 0 & 0 & 0 & 0 & 0 & 0 & 1 & 0 & 0 & 0 & 0 & 0 & 0 & 0 & 0 & 0 & 0 & 0 & 0 & 0 \\
\hline
\end{tabular}

Ethers

\begin{tabular}{|c|c|c|c|c|c|c|c|c|c|c|c|c|c|c|c|c|c|c|c|c|c|c|c|c|}
\hline L-1 & 2 & 1 & 0 & 0 & 0 & 0 & 0 & 0 & 0 & 0 & 0 & 0 & 0 & 0 & 0 & 0 & 0 & 0 & 0 & 0 & 0 & 1 & 0 & 0 \\
\hline L-2 & 4 & 1 & 2 & 0 & 0 & 0 & 0 & 0 & 0 & 0 & 0 & 0 & 0 & 0 & 0 & 0 & 0 & 0 & 0 & 0 & 0 & 1 & 0 & 0 \\
\hline L-3 & 4 & 2 & 1 & 0 & 0 & 0 & 0 & 0 & 0 & 0 & 0 & 0 & 0 & 0 & 0 & 0 & 0 & 0 & 0 & 0 & 0 & 0 & 1 & 0 \\
\hline L-4 & 5 & 3 & 0 & 0 & 1 & 0 & 0 & 0 & 0 & 0 & 0 & 0 & 0 & 0 & 0 & 0 & 0 & 0 & 0 & 0 & 0 & 1 & 0 & 0 \\
\hline L-5 & 6 & 2 & 3 & 0 & 0 & 0 & 0 & 0 & 0 & 0 & 0 & 0 & 0 & 0 & 0 & 0 & 0 & 0 & 0 & 0 & 0 & 0 & 1 & 0 \\
\hline L-6 & 6 & 2 & 3 & 0 & 0 & 0 & 0 & 0 & 0 & 0 & 0 & 0 & 0 & 0 & 0 & 0 & 0 & 0 & 0 & 0 & 0 & 0 & 1 & 0 \\
\hline L-7 & 8 & 2 & 5 & 0 & 0 & 0 & 0 & 0 & 0 & 0 & 0 & 0 & 0 & 0 & 0 & 0 & 0 & 0 & 0 & 0 & 0 & 0 & 1 & 0 \\
\hline L-8 & 10 & 2 & 7 & 0 & 0 & 0 & 0 & 0 & 0 & 0 & 0 & 0 & 0 & 0 & 0 & 0 & 0 & 0 & 0 & 0 & 0 & 0 & 1 & 0 \\
\hline L-9 & 13 & 0 & 0 & 0 & 0 & 0 & 0 & 0 & 0 & 0 & 0 & 0 & 0 & 0 & 0 & 0 & 0 & 0 & 0 & 10 & 2 & 0 & 0 & 1 \\
\hline L-10 & 7 & 2 & 4 & 0 & 0 & 0 & 0 & 0 & 0 & 0 & 0 & 0 & 0 & 0 & 0 & 0 & 0 & 0 & 0 & 0 & 0 & 0 & 1 & 0 \\
\hline L-11 & 10 & 4 & 3 & 2 & 0 & 0 & 0 & 0 & 0 & 0 & 0 & 0 & 0 & 0 & 0 & 0 & 0 & 0 & 0 & 0 & 0 & 0 & 1 & 0 \\
\hline L-12 & 12 & 2 & 9 & 0 & 0 & 0 & 0 & 0 & 0 & 0 & 0 & 0 & 0 & 0 & 0 & 0 & 0 & 0 & 0 & 0 & 0 & 0 & 1 & 0 \\
\hline L-13 & 14 & 2 & 11 & 0 & 0 & 0 & 0 & 0 & 0 & 0 & 0 & 0 & 0 & 0 & 0 & 0 & 0 & 0 & 0 & 0 & 0 & 0 & 1 & 0 \\
\hline L-14 & 16 & 2 & 13 & 0 & 0 & 0 & 0 & 0 & 0 & 0 & 0 & 0 & 0 & 0 & 0 & 0 & 0 & 0 & 0 & 0 & 0 & 0 & 1 & 0 \\
\hline
\end{tabular}


Table - continued from previous page

\begin{tabular}{|c|c|c|c|c|c|c|c|c|c|c|c|c|c|c|c|c|c|c|c|c|c|c|c|c|}
\hline SI-Sybmol & $n^{t}$ & $\stackrel{\infty}{O}$ & 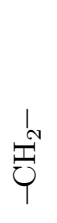 & $\begin{array}{l}\stackrel{V}{\Xi} \\
\stackrel{\Xi}{O}\end{array}$ & $\begin{array}{l}\vee \\
U \\
\wedge\end{array}$ & $\underbrace{\|}_{\mathcal{N}}$ & 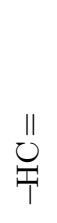 & $\underset{\wedge}{\|}$ & $\begin{array}{l}0 \\
\| \\
\cup \\
\wedge\end{array}$ & $\begin{array}{l}0 \\
\| \\
\stackrel{P}{O}\end{array}$ & $\stackrel{1}{9}$ & $\underset{i}{\mathbb{Z}}$ & $\underset{\wedge}{\stackrel{\text { 党 }}{\wedge}}$ & $\begin{array}{l}\circlearrowright \\
\circlearrowright \\
\circlearrowright \\
ٍ\end{array}$ & \begin{tabular}{l}
1 \\
0 \\
\hdashline
\end{tabular} & 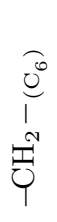 & $\begin{array}{l}\overbrace{}^{0} \\
\vec{V} \\
\vec{U}\end{array}$ & $\begin{array}{l}\overbrace{}^{10} \\
\underbrace{\prime} \\
\underbrace{N}\end{array}$ & 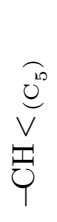 & 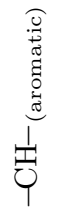 & 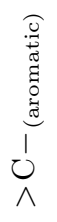 & $\begin{array}{l}\infty \\
0 \\
0 \\
0 \\
0\end{array}$ & 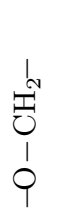 & 1 \\
\hline L-15 & 12 & 1 & 1 & 0 & 0 & 1 & 1 & 0 & 0 & 0 & 1 & 0 & 0 & 0 & 0 & 0 & 0 & 0 & 0 & 3 & 3 & 0 & 0 & 1 \\
\hline
\end{tabular}

contains Nitrogen

\begin{tabular}{|c|c|c|c|c|c|c|c|c|c|c|c|c|c|c|c|c|c|c|c|c|c|c|c|c|}
\hline M-1 & 2 & 1 & 0 & 0 & 0 & 0 & 0 & 0 & 0 & 0 & 0 & 1 & 0 & 0 & 0 & 0 & 0 & 0 & 0 & 0 & 0 & 0 & 0 & 0 \\
\hline M-2 & 4 & 2 & 0 & 1 & 0 & 0 & 0 & 0 & 0 & 0 & 0 & 1 & 0 & 0 & 0 & 0 & 0 & 0 & 0 & 0 & 0 & 0 & 0 & 0 \\
\hline M-3 & 5 & 2 & 2 & 0 & 0 & 0 & 0 & 0 & 0 & 0 & 0 & 0 & 1 & 0 & 0 & 0 & 0 & 0 & 0 & 0 & 0 & 0 & 0 & 0 \\
\hline M-4 & 7 & 1 & 5 & 0 & 0 & 0 & 0 & 0 & 0 & 0 & 0 & 1 & 0 & 0 & 0 & 0 & 0 & 0 & 0 & 0 & 0 & 0 & 0 & 0 \\
\hline M-5 & 9 & 1 & 7 & 0 & 0 & 0 & 0 & 0 & 0 & 0 & 0 & 1 & 0 & 0 & 0 & 0 & 0 & 0 & 0 & 0 & 0 & 0 & 0 & 0 \\
\hline M-6 & 9 & 2 & 6 & 0 & 0 & 0 & 0 & 0 & 0 & 0 & 0 & 0 & 1 & 0 & 0 & 0 & 0 & 0 & 0 & 0 & 0 & 0 & 0 & 0 \\
\hline M-7 & 7 & 4 & 0 & 2 & 0 & 0 & 0 & 0 & 0 & 0 & 0 & 0 & 1 & 0 & 0 & 0 & 0 & 0 & 0 & 0 & 0 & 0 & 0 & 0 \\
\hline \multicolumn{25}{|c|}{ multiple functional groups } \\
\hline O-1 & 4 & 0 & 2 & 0 & 0 & 0 & 0 & 0 & 0 & 0 & 2 & 0 & 0 & 0 & 0 & 0 & 0 & 0 & 0 & 0 & 0 & 0 & 0 & 0 \\
\hline $\mathrm{O}-2$ & 5 & 0 & 3 & 0 & 0 & 0 & 0 & 0 & 0 & 0 & 2 & 0 & 0 & 0 & 0 & 0 & 0 & 0 & 0 & 0 & 0 & 0 & 0 & 0 \\
\hline O-3 & 5 & 1 & 1 & 1 & 0 & 0 & 0 & 0 & 0 & 0 & 2 & 0 & 0 & 0 & 0 & 0 & 0 & 0 & 0 & 0 & 0 & 0 & 0 & 0 \\
\hline $\mathrm{O}-4$ & 6 & 1 & 2 & 1 & 0 & 0 & 0 & 0 & 0 & 0 & 2 & 0 & 0 & 0 & 0 & 0 & 0 & 0 & 0 & 0 & 0 & 0 & 0 & 0 \\
\hline O-5 & 6 & 0 & 4 & 0 & 0 & 0 & 0 & 0 & 0 & 0 & 2 & 0 & 0 & 0 & 0 & 0 & 0 & 0 & 0 & 0 & 0 & 0 & 0 & 0 \\
\hline O-6 & 7 & 2 & 2 & 0 & 1 & 0 & 0 & 0 & 0 & 0 & 2 & 0 & 0 & 0 & 0 & 0 & 0 & 0 & 0 & 0 & 0 & 0 & 0 & 0 \\
\hline O-7 & 7 & 0 & 5 & 0 & 0 & 0 & 0 & 0 & 0 & 0 & 2 & 0 & 0 & 0 & 0 & 0 & 0 & 0 & 0 & 0 & 0 & 0 & 0 & 0 \\
\hline O-8 & 8 & 0 & 6 & 0 & 0 & 0 & 0 & 0 & 0 & 0 & 2 & 0 & 0 & 0 & 0 & 0 & 0 & 0 & 0 & 0 & 0 & 0 & 0 & 0 \\
\hline O-9 & 10 & 0 & 8 & 0 & 0 & 0 & 0 & 0 & 0 & 0 & 2 & 0 & 0 & 0 & 0 & 0 & 0 & 0 & 0 & 0 & 0 & 0 & 0 & 0 \\
\hline O-10 & 11 & 0 & 9 & 0 & 0 & 0 & 0 & 0 & 0 & 0 & 2 & 0 & 0 & 0 & 0 & 0 & 0 & 0 & 0 & 0 & 0 & 0 & 0 & 0 \\
\hline O-11 & 12 & 0 & 10 & 0 & 0 & 0 & 0 & 0 & 0 & 0 & 2 & 0 & 0 & 0 & 0 & 0 & 0 & 0 & 0 & 0 & 0 & 0 & 0 & 0 \\
\hline O-12 & 6 & 0 & 2 & 1 & 0 & 0 & 0 & 0 & 0 & 0 & 3 & 0 & 0 & 0 & 0 & 0 & 0 & 0 & 0 & 0 & 0 & 0 & 0 & 0 \\
\hline O-13 & 8 & 1 & 3 & 0 & 1 & 0 & 0 & 0 & 0 & 0 & 3 & 0 & 0 & 0 & 0 & 0 & 0 & 0 & 0 & 0 & 0 & 0 & 0 & 0 \\
\hline O-14 & 9 & 1 & 4 & 0 & 1 & 0 & 0 & 0 & 0 & 0 & 3 & 0 & 0 & 0 & 0 & 0 & 0 & 0 & 0 & 0 & 0 & 0 & 0 & 0 \\
\hline O-15 & 12 & 0 & 0 & 0 & 0 & 0 & 0 & 0 & 0 & 0 & 0 & 0 & 0 & 0 & 0 & 0 & 0 & 0 & 0 & 10 & 2 & 0 & 0 & 0 \\
\hline O-16 & 13 & 0 & 1 & 0 & 0 & 0 & 0 & 0 & 0 & 0 & 0 & 0 & 0 & 0 & 0 & 0 & 0 & 0 & 0 & 10 & 2 & 0 & 0 & 0 \\
\hline O-17 & 15 & 2 & 1 & 0 & 0 & 0 & 0 & 0 & 0 & 0 & 0 & 0 & 0 & 0 & 0 & 0 & 0 & 0 & 0 & 8 & 4 & 0 & 0 & 0 \\
\hline O-18 & 15 & 2 & 0 & 1 & 0 & 0 & 0 & 0 & 0 & 0 & 0 & 0 & 0 & 0 & 0 & 0 & 0 & 0 & 0 & 9 & 3 & 0 & 0 & 0 \\
\hline O-19 & 19 & 4 & 1 & 2 & 0 & 0 & 0 & 0 & 0 & 0 & 0 & 0 & 0 & 0 & 0 & 0 & 0 & 0 & 0 & 8 & 4 & 0 & 0 & 0 \\
\hline O-20 & 18 & 0 & 0 & 0 & 0 & 0 & 0 & 0 & 0 & 0 & 0 & 0 & 0 & 0 & 0 & 0 & 0 & 0 & 0 & 14 & 4 & 0 & 0 & 0 \\
\hline O-21 & 18 & 0 & 0 & 0 & 0 & 0 & 0 & 0 & 0 & 0 & 0 & 0 & 0 & 0 & 0 & 0 & 0 & 0 & 0 & 14 & 4 & 0 & 0 & 0 \\
\hline
\end{tabular}


Table - continued from previous page

\begin{tabular}{|c|c|c|c|c|c|c|c|c|c|c|c|c|c|c|c|c|c|c|c|c|c|c|c|c|}
\hline SI-Sybmol & $n^{t}$ & $\stackrel{\infty}{\mathcal{O}}$ & 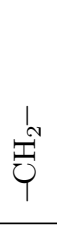 & $\begin{array}{l}V \\
\stackrel{\nabla}{\mho}\end{array}$ & $\begin{array}{l}\vee \\
U \\
\wedge\end{array}$ & $\begin{array}{l}\| \\
\bigcup_{N} \\
\mathcal{I}^{\prime}\end{array}$ & $\underset{⿱ ⺌}{\circlearrowright}$ & $\begin{array}{l}\| \\
\circlearrowright \\
\wedge\end{array}$ & $\begin{array}{l}0 \\
\| \\
\circlearrowright \\
\wedge\end{array}$ & 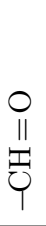 & $\stackrel{T}{0}$ & $\underset{i}{\mathbf{Z}_{1}^{N}}$ & $\stackrel{\text { 叓 }}{\wedge}$ & 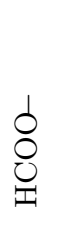 & \begin{tabular}{l} 
ঠ \\
$ٍ$ \\
\hdashline
\end{tabular} & 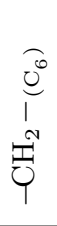 & $\begin{array}{l}\overbrace{}^{0} \\
\stackrel{V}{V} \\
\stackrel{O}{U}\end{array}$ & 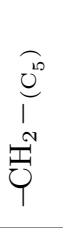 & 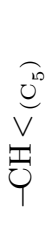 & 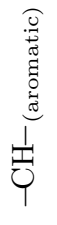 & 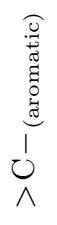 & $\begin{array}{c}\mathscr{S}_{0}^{\infty} \\
1 \\
0\end{array}$ & 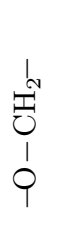 & $\phi$ \\
\hline O-22 & 18 & 0 & 0 & 0 & 0 & 0 & 0 & 0 & 0 & 0 & 0 & 0 & 0 & 0 & 0 & 0 & 0 & 0 & 0 & 14 & 4 & 0 & 0 & 0 \\
\hline O-23 & 10 & 0 & 0 & 0 & 0 & 0 & 0 & 0 & 0 & 0 & 0 & 0 & 0 & 0 & 0 & 0 & 0 & 8 & 2 & 0 & 0 & 0 & 0 & 0 \\
\hline O-24 & 12 & 0 & 0 & 0 & 0 & 0 & 0 & 0 & 0 & 0 & 0 & 0 & 0 & 0 & 0 & 10 & 2 & 0 & 0 & 0 & 0 & 0 & 0 & 0 \\
\hline O-25 & 13 & 0 & 1 & 0 & 0 & 0 & 0 & 0 & 0 & 0 & 0 & 0 & 0 & 0 & 0 & 10 & 2 & 0 & 0 & 0 & 0 & 0 & 0 & 0 \\
\hline O-26 & 19 & 1 & 5 & 1 & 0 & 0 & 0 & 0 & 0 & 0 & 0 & 0 & 0 & 0 & 0 & 10 & 2 & 0 & 0 & 0 & 0 & 0 & 0 & 0 \\
\hline O-27 & 8 & 2 & 4 & 0 & 0 & 0 & 0 & 0 & 0 & 0 & 0 & 0 & 0 & 0 & 2 & 0 & 0 & 0 & 0 & 0 & 0 & 0 & 0 & 0 \\
\hline O-28 & 10 & 2 & 6 & 0 & 0 & 0 & 0 & 0 & 0 & 0 & 0 & 0 & 0 & 0 & 2 & 0 & 0 & 0 & 0 & 0 & 0 & 0 & 0 & 0 \\
\hline O-29 & 18 & 2 & 14 & 0 & 0 & 0 & 0 & 0 & 0 & 0 & 0 & 0 & 0 & 0 & 2 & 0 & 0 & 0 & 0 & 0 & 0 & 0 & 0 & 0 \\
\hline O-30 & 18 & 2 & 14 & 0 & 0 & 0 & 0 & 0 & 0 & 0 & 0 & 0 & 0 & 0 & 2 & 0 & 0 & 0 & 0 & 0 & 0 & 0 & 0 & 0 \\
\hline O-31 & 18 & 4 & 10 & 2 & 0 & 0 & 0 & 0 & 0 & 0 & 0 & 0 & 0 & 0 & 2 & 0 & 0 & 0 & 0 & 0 & 0 & 0 & 0 & 0 \\
\hline O-32 & 20 & 2 & 16 & 0 & 0 & 0 & 0 & 0 & 0 & 0 & 0 & 0 & 0 & 0 & 2 & 0 & 0 & 0 & 0 & 0 & 0 & 0 & 0 & 0 \\
\hline O-33 & 22 & 4 & 14 & 2 & 0 & 0 & 0 & 0 & 0 & 0 & 0 & 0 & 0 & 0 & 2 & 0 & 0 & 0 & 0 & 0 & 0 & 0 & 0 & 0 \\
\hline O-34 & 22 & 2 & 18 & 0 & 0 & 0 & 0 & 0 & 0 & 0 & 0 & 0 & 0 & 0 & 2 & 0 & 0 & 0 & 0 & 0 & 0 & 0 & 0 & 0 \\
\hline O-35 & 22 & 2 & 18 & 0 & 0 & 0 & 0 & 0 & 0 & 0 & 0 & 0 & 0 & 0 & 2 & 0 & 0 & 0 & 0 & 0 & 0 & 0 & 0 & 0 \\
\hline O-36 & 3 & 0 & 1 & 0 & 0 & 0 & 0 & 0 & 0 & 0 & 0 & 0 & 0 & 0 & 0 & 0 & 0 & 0 & 0 & 0 & 0 & 2 & 0 & 0 \\
\hline O-37 & 4 & 0 & 2 & 0 & 0 & 0 & 0 & 0 & 0 & 0 & 0 & 0 & 0 & 0 & 0 & 0 & 0 & 0 & 0 & 0 & 0 & 2 & 0 & 0 \\
\hline O-38 & 6 & 2 & 2 & 0 & 0 & 0 & 0 & 0 & 0 & 0 & 0 & 0 & 0 & 0 & 0 & 0 & 0 & 0 & 0 & 0 & 0 & 0 & 2 & 0 \\
\hline O-39 & 9 & 2 & 5 & 0 & 0 & 0 & 0 & 0 & 0 & 0 & 0 & 0 & 0 & 0 & 0 & 0 & 0 & 0 & 0 & 0 & 0 & 0 & 2 & 0 \\
\hline $\mathrm{O}-40$ & 10 & 2 & 6 & 0 & 0 & 0 & 0 & 0 & 0 & 0 & 0 & 0 & 0 & 0 & 0 & 0 & 0 & 0 & 0 & 0 & 0 & 0 & 2 & 0 \\
\hline O-41 & 6 & 0 & 3 & 0 & 0 & 0 & 0 & 0 & 0 & 0 & 0 & 0 & 0 & 0 & 0 & 0 & 0 & 0 & 0 & 0 & 0 & 2 & 1 & 0 \\
\hline O- 42 & 8 & 2 & 3 & 0 & 0 & 0 & 0 & 0 & 0 & 0 & 0 & 0 & 0 & 0 & 0 & 0 & 0 & 0 & 0 & 0 & 0 & 0 & 3 & 0 \\
\hline O-43 & 12 & 2 & 7 & 0 & 0 & 0 & 0 & 0 & 0 & 0 & 0 & 0 & 0 & 0 & 0 & 0 & 0 & 0 & 0 & 0 & 0 & 0 & 3 & 0 \\
\hline $\mathrm{O}-44$ & 8 & 0 & 4 & 0 & 0 & 0 & 0 & 0 & 0 & 0 & 0 & 0 & 0 & 0 & 0 & 0 & 0 & 0 & 0 & 0 & 0 & 2 & 2 & 0 \\
\hline O-45 & 10 & 0 & 5 & 0 & 0 & 0 & 0 & 0 & 0 & 0 & 0 & 0 & 0 & 0 & 0 & 0 & 0 & 0 & 0 & 0 & 0 & 2 & 3 & 0 \\
\hline
\end{tabular}

mixed functional groups, each group only once

\begin{tabular}{|c|c|c|c|c|c|c|c|c|c|c|c|c|c|c|c|c|c|c|c|c|c|c|c|c|}
\hline P-1 & 21 & 5 & 9 & 3 & 1 & 1 & 1 & 0 & 0 & 0 & 1 & 0 & 0 & 0 & 0 & 0 & 0 & 0 & 0 & 0 & 0 & 0 & 0 & 0 \\
\hline P-2 & 7 & 0 & 0 & 0 & 0 & 0 & 0 & 0 & 0 & 0 & 1 & 0 & 0 & 0 & 0 & 0 & 0 & 0 & 0 & 5 & 1 & 0 & 0 & 0 \\
\hline P-3 & 8 & 0 & 1 & 0 & 0 & 0 & 0 & 0 & 0 & 0 & 1 & 0 & 0 & 0 & 0 & 0 & 0 & 0 & 0 & 5 & 1 & 0 & 0 & 0 \\
\hline $\mathrm{P}-4$ & 8 & 1 & 0 & 0 & 0 & 0 & 0 & 0 & 0 & 0 & 1 & 0 & 0 & 0 & 0 & 0 & 0 & 0 & 0 & 4 & 2 & 0 & 0 & 0 \\
\hline P-5 & 6 & 0 & 0 & 0 & 0 & 0 & 0 & 0 & 0 & 0 & 1 & 0 & 0 & 0 & 0 & 0 & 0 & 4 & 1 & 0 & 0 & 0 & 0 & 0 \\
\hline $\mathrm{P}-6$ & 7 & 0 & 0 & 0 & 0 & 0 & 0 & 0 & 0 & 0 & 1 & 0 & 0 & 0 & 0 & 5 & 1 & 0 & 0 & 0 & 0 & 0 & 0 & 0 \\
\hline
\end{tabular}


Table - continued from previous page

\begin{tabular}{|c|c|c|c|c|c|c|c|c|c|c|c|c|c|c|c|c|c|c|c|c|c|c|c|c|}
\hline SI-Sybmol & $n^{t}$ & $\stackrel{\infty}{\mathcal{O}}$ & $\underset{\mathrm{T}}{\mathrm{I}_{N}}$ & $\begin{array}{l}V \\
\text { 至 }\end{array}$ & $\begin{array}{l}\vee \\
\cup \\
\wedge\end{array}$ & $\begin{array}{l}\| \\
\bigcup_{N} \\
\mathbb{F}^{\prime}\end{array}$ & 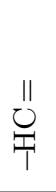 & $\begin{array}{l}\| \\
\circlearrowright \\
\wedge\end{array}$ & $\begin{array}{l}0 \\
\| \\
0 \\
\wedge\end{array}$ & 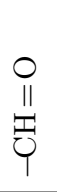 & 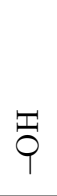 & $\underset{T}{\mathbb{N}}$ & $\stackrel{\text { 党 }}{\wedge}$ & $\begin{array}{l}d \\
8 \\
0 \\
0 \\
\end{array}$ & \begin{tabular}{l} 
d \\
0 \\
\hdashline
\end{tabular} & $\begin{array}{l}\tilde{0}^{0} \\
1 \\
\mathbb{1} \\
\overrightarrow{0}\end{array}$ & 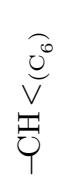 & 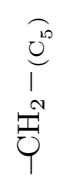 & 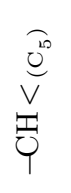 & 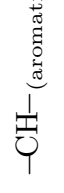 & 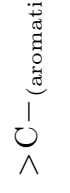 & $\begin{array}{c}\Xi_{0}^{\infty} \\
\text { I } \\
0\end{array}$ & $\begin{array}{l}I_{N} \\
\underset{0}{0} \\
1 \\
0\end{array}$ & p \\
\hline P-7 & 4 & 0 & 2 & 0 & 0 & 0 & 0 & 0 & 0 & 0 & 1 & 0 & 0 & 0 & 0 & 0 & 0 & 0 & 0 & 0 & 0 & 1 & 0 & 0 \\
\hline P-8 & 5 & 1 & 2 & 0 & 0 & 0 & 0 & 0 & 0 & 0 & 1 & 0 & 0 & 0 & 0 & 0 & 0 & 0 & 0 & 0 & 0 & 0 & 1 & 0 \\
\hline P-9 & 7 & 1 & 4 & 0 & 0 & 0 & 0 & 0 & 0 & 0 & 1 & 0 & 0 & 0 & 0 & 0 & 0 & 0 & 0 & 0 & 0 & 0 & 1 & 0 \\
\hline P-10 & 6 & 0 & 3 & 0 & 0 & 0 & 0 & 0 & 0 & 0 & 1 & 0 & 0 & 0 & 0 & 0 & 0 & 0 & 0 & 0 & 0 & 1 & 1 & 0 \\
\hline P-11 & 9 & 0 & 1 & 0 & 0 & 0 & 0 & 0 & 0 & 0 & 1 & 0 & 0 & 0 & 0 & 0 & 0 & 0 & 0 & 5 & 1 & 0 & 1 & 0 \\
\hline $\mathrm{P}-12$ & 9 & 1 & 6 & 0 & 0 & 0 & 0 & 0 & 0 & 0 & 1 & 0 & 0 & 0 & 0 & 0 & 0 & 0 & 0 & 0 & 0 & 0 & 1 & 0 \\
\hline P-13 & 5 & 1 & 1 & 0 & 1 & 1 & 0 & 0 & 0 & 1 & 0 & 0 & 0 & 0 & 0 & 0 & 0 & 0 & 0 & 0 & 0 & 0 & 0 & 0 \\
\hline P-14 & 7 & 0 & 0 & 0 & 0 & 0 & 0 & 0 & 0 & 1 & 0 & 0 & 0 & 0 & 0 & 0 & 0 & 0 & 0 & 5 & 1 & 0 & 0 & 0 \\
\hline P-15 & 5 & 0 & 0 & 0 & 0 & 0 & 2 & 0 & 0 & 0 & 0 & 0 & 0 & 0 & 0 & 0 & 0 & 3 & 0 & 0 & 0 & 0 & 0 & 0 \\
\hline P-16 & 6 & 0 & 0 & 0 & 0 & 0 & 2 & 0 & 0 & 0 & 0 & 0 & 0 & 0 & 0 & 4 & 0 & 0 & 0 & 0 & 0 & 0 & 0 & 0 \\
\hline P-17 & 7 & 1 & 1 & 0 & 0 & 0 & 0 & 1 & 0 & 0 & 0 & 0 & 0 & 0 & 0 & 0 & 0 & 4 & 0 & 0 & 0 & 0 & 0 & 0 \\
\hline P-18 & 8 & 0 & 0 & 0 & 0 & 1 & 6 & 0 & 0 & 0 & 0 & 0 & 0 & 0 & 0 & 0 & 0 & 0 & 0 & 5 & 1 & 0 & 0 & 0 \\
\hline P-19 & 13 & 1 & 4 & 0 & 0 & 0 & 0 & 0 & 0 & 0 & 1 & 0 & 0 & 0 & 1 & 0 & 0 & 0 & 0 & 4 & 2 & 0 & 0 & 0 \\
\hline P-20 & 4 & 1 & 0 & 0 & 0 & 1 & 1 & 0 & 0 & 0 & 0 & 0 & 0 & 0 & 1 & 0 & 0 & 0 & 0 & 0 & 0 & 0 & 0 & 0 \\
\hline P-21 & 5 & 2 & 0 & 0 & 0 & 0 & 2 & 0 & 0 & 0 & 0 & 0 & 0 & 0 & 1 & 0 & 0 & 0 & 0 & 0 & 0 & 0 & 0 & 0 \\
\hline P-22 & 5 & 2 & 0 & 0 & 0 & 1 & 0 & 1 & 0 & 0 & 0 & 0 & 0 & 0 & 1 & 0 & 0 & 0 & 0 & 0 & 0 & 0 & 0 & 0 \\
\hline P-23 & 6 & 1 & 2 & 0 & 0 & 0 & 2 & 0 & 0 & 0 & 0 & 0 & 0 & 0 & 1 & 0 & 0 & 0 & 0 & 0 & 0 & 0 & 0 & 0 \\
\hline P-24 & 19 & 2 & 14 & 0 & 0 & 0 & 2 & 0 & 0 & 0 & 0 & 0 & 0 & 0 & 1 & 0 & 0 & 0 & 0 & 0 & 0 & 0 & 0 & 0 \\
\hline P-25 & 8 & 1 & 0 & 0 & 0 & 0 & 0 & 0 & 0 & 0 & 0 & 0 & 0 & 0 & 1 & 0 & 0 & 0 & 0 & 4 & 2 & 0 & 0 & 0 \\
\hline P-26 & 9 & 1 & 1 & 0 & 0 & 0 & 0 & 0 & 0 & 0 & 0 & 0 & 0 & 0 & 1 & 0 & 0 & 0 & 0 & 5 & 1 & 0 & 0 & 0 \\
\hline P-27 & 9 & 1 & 0 & 0 & 0 & 0 & 0 & 0 & 0 & 0 & 1 & 0 & 0 & 0 & 1 & 0 & 0 & 0 & 0 & 4 & 2 & 0 & 0 & 0 \\
\hline P-28 & 10 & 1 & 2 & 0 & 0 & 0 & 0 & 0 & 0 & 0 & 0 & 0 & 0 & 0 & 1 & 0 & 0 & 0 & 0 & 5 & 1 & 0 & 0 & 0 \\
\hline P-29 & 3 & 1 & 0 & 0 & 0 & 0 & 0 & 0 & 0 & 0 & 0 & 0 & 0 & 0 & 1 & 0 & 0 & 0 & 0 & 0 & 0 & 1 & 0 & 0 \\
\hline P-30 & 5 & 1 & 2 & 0 & 0 & 0 & 0 & 0 & 0 & 0 & 0 & 0 & 0 & 0 & 1 & 0 & 0 & 0 & 0 & 0 & 0 & 0 & 1 & 0 \\
\hline P-31 & 5 & 1 & 2 & 0 & 0 & 0 & 0 & 0 & 0 & 0 & 0 & 0 & 0 & 0 & 1 & 0 & 0 & 0 & 0 & 0 & 0 & 0 & 1 & 0 \\
\hline P-32 & 9 & 2 & 4 & 1 & 0 & 0 & 0 & 0 & 0 & 0 & 1 & 0 & 0 & 0 & 0 & 0 & 0 & 0 & 0 & 0 & 0 & 0 & 1 & 0 \\
\hline P-33 & 9 & 1 & 0 & 0 & 0 & 0 & 0 & 0 & 1 & 0 & 1 & 0 & 0 & 0 & 0 & 0 & 0 & 0 & 0 & 4 & 2 & 0 & 0 & 0 \\
\hline P-34 & 8 & 1 & 0 & 0 & 0 & 0 & 0 & 0 & 1 & 0 & 0 & 0 & 0 & 0 & 0 & 0 & 0 & 0 & 0 & 5 & 1 & 0 & 0 & 0 \\
\hline P-35 & 9 & 2 & 0 & 0 & 0 & 0 & 0 & 0 & 1 & 0 & 0 & 0 & 0 & 0 & 0 & 0 & 0 & 0 & 0 & 4 & 2 & 0 & 0 & 0 \\
\hline P-36 & 5 & 0 & 0 & 0 & 0 & 0 & 0 & 0 & 1 & 0 & 0 & 0 & 0 & 0 & 0 & 0 & 0 & 4 & 0 & 0 & 0 & 0 & 0 & 0 \\
\hline P-37 & 6 & 0 & 0 & 0 & 0 & 0 & 0 & 0 & 1 & 0 & 0 & 0 & 0 & 0 & 0 & 5 & 0 & 0 & 0 & 0 & 0 & 0 & 0 & 0 \\
\hline
\end{tabular}


Table - continued from previous page

\begin{tabular}{|c|c|c|c|c|c|c|c|c|c|c|c|c|c|c|c|c|c|c|c|c|c|c|c|c|}
\hline SI-Sybmol & $n^{t}$ & $\stackrel{\oplus}{\ddot{O}}$ & 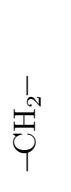 & 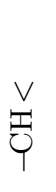 & $\begin{array}{l}\vee \\
U \\
\wedge\end{array}$ & $\begin{array}{l}\| \\
U_{N} \\
\mathbb{J}^{N}\end{array}$ & $\underset{⿱ ⺌}{\bigcup_{T}^{\prime}}$ & $\begin{array}{l}\| \\
\circlearrowright \\
\wedge\end{array}$ & $\begin{array}{l}0 \\
\| \\
0 \\
\wedge\end{array}$ & $\begin{array}{l}0 \\
\| \\
\stackrel{1}{O} \\
\end{array}$ & ज्ञ & 空 & $\underset{\wedge}{\stackrel{\mathrm{Z}}{\mathrm{Z}}}$ & $\begin{array}{l}\text { d } \\
\bigcirc \\
\circlearrowright \\
\text { 出 }\end{array}$ & \begin{tabular}{l} 
d \\
0 \\
\hdashline
\end{tabular} & 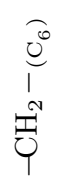 & $\begin{array}{l}\widehat{O} \\
\stackrel{0}{V} \\
\vec{U} \\
\dot{Y}\end{array}$ & 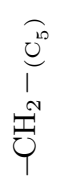 & 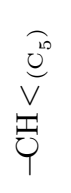 & 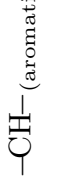 & 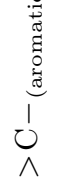 & $\begin{array}{l}\stackrel{\infty}{0} \\
0 \\
1 \\
0\end{array}$ & $\begin{array}{l}I_{N} \\
\underset{0}{0} \\
1 \\
0\end{array}$ & $\phi$ \\
\hline P-38 & 4 & 2 & 0 & 0 & 0 & 0 & 0 & 0 & 1 & 0 & 0 & 0 & 0 & 0 & 1 & 0 & 0 & 0 & 0 & 0 & 0 & 0 & 0 & 0 \\
\hline P-39 & 4 & 0 & 2 & 0 & 0 & 0 & 0 & 0 & 0 & 0 & 1 & 1 & 0 & 0 & 0 & 0 & 0 & 0 & 0 & 0 & 0 & 0 & 0 & 0 \\
\hline P-40 & 2 & 0 & 0 & 0 & 0 & 0 & 0 & 0 & 0 & 1 & 0 & 1 & 0 & 0 & 0 & 0 & 0 & 0 & 0 & 0 & 0 & 0 & 0 & 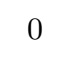 \\
\hline P-41 & 7 & 0 & 0 & 0 & 0 & 0 & 0 & 0 & 0 & 0 & 0 & 1 & 0 & 0 & 0 & 0 & 0 & 0 & 0 & 5 & 1 & 0 & 0 & 0 \\
\hline P-42 & 6 & 0 & 0 & 0 & 0 & 0 & 0 & 0 & 0 & 0 & 0 & 0 & 1 & 0 & 0 & 5 & 0 & 0 & 0 & 0 & 0 & 0 & 0 & 0 \\
\hline P-43 & 7 & 0 & 0 & 0 & 0 & 0 & 0 & 0 & 0 & 0 & 0 & 1 & 0 & 0 & 0 & 5 & 1 & 0 & 0 & 0 & 0 & 0 & 0 & 0 \\
\hline
\end{tabular}

\section{mixed functional groups, each group maximum twice}

\begin{tabular}{|c|c|c|c|c|c|c|c|c|c|c|c|c|c|c|c|c|c|c|c|c|c|c|c|c|}
\hline Q-1 & 13 & 0 & 0 & 0 & 0 & 0 & 0 & 0 & 0 & 0 & 1 & 0 & 0 & 0 & 0 & 0 & 0 & 0 & 0 & 9 & 3 & 0 & 0 & 0 \\
\hline Q-2 & 6 & 0 & 3 & 0 & 0 & 0 & 0 & 0 & 0 & 0 & 2 & 0 & 0 & 0 & 0 & 0 & 0 & 0 & 0 & 0 & 0 & 0 & 1 & 0 \\
\hline Q-3 & 7 & 1 & 3 & 0 & 0 & 0 & 0 & 0 & 0 & 0 & 1 & 0 & 0 & 0 & 0 & 0 & 0 & 0 & 0 & 0 & 0 & 0 & 2 & 0 \\
\hline Q-4 & 8 & 0 & 4 & 0 & 0 & 0 & 0 & 0 & 0 & 0 & 2 & 0 & 0 & 0 & 0 & 0 & 0 & 0 & 0 & 0 & 0 & 0 & 2 & 0 \\
\hline Q-1 & 9 & 1 & 5 & 0 & 0 & 0 & 0 & 0 & 0 & 0 & 1 & 0 & 0 & 0 & 0 & 0 & 0 & 0 & 0 & 0 & 0 & 0 & 2 & 0 \\
\hline Q-2 & 7 & 0 & 4 & 0 & 0 & 0 & 0 & 0 & 0 & 0 & 2 & 0 & 1 & 0 & 0 & 0 & 0 & 0 & 0 & 0 & 0 & 0 & 0 & 0 \\
\hline Q-3 & 16 & 2 & 10 & 0 & 0 & 0 & 2 & 0 & 0 & 0 & 0 & 0 & 0 & 0 & 2 & 0 & 0 & 0 & 0 & 0 & 0 & 0 & 0 & 0 \\
\hline Q-4 & 19 & 2 & 12 & 0 & 0 & 0 & 4 & 0 & 0 & 0 & 0 & 0 & 0 & 0 & 1 & 0 & 0 & 0 & 0 & 0 & 0 & 0 & 0 & 0 \\
\hline Q-1 & 18 & 2 & 12 & 0 & 0 & 0 & 2 & 0 & 0 & 0 & 0 & 0 & 0 & 0 & 2 & 0 & 0 & 0 & 0 & 0 & 0 & 0 & 0 & 0 \\
\hline Q-2 & 20 & 2 & 14 & 0 & 0 & 0 & 2 & 0 & 0 & 0 & 0 & 0 & 0 & 0 & 2 & 0 & 0 & 0 & 0 & 0 & 0 & 0 & 0 & 0 \\
\hline Q-3 & 22 & 2 & 16 & 0 & 0 & 0 & 2 & 0 & 0 & 0 & 0 & 0 & 0 & 0 & 2 & 0 & 0 & 0 & 0 & 0 & 0 & 0 & 0 & 0 \\
\hline Q-4 & 10 & 2 & 0 & 0 & 0 & 0 & 0 & 0 & 0 & 0 & 0 & 0 & 0 & 0 & 2 & 0 & 0 & 0 & 0 & 4 & 2 & 0 & 0 & 0 \\
\hline Q-1 & 10 & 2 & 0 & 0 & 0 & 0 & 0 & 0 & 0 & 0 & 0 & 0 & 0 & 0 & 2 & 0 & 0 & 0 & 0 & 4 & 2 & 0 & 0 & 0 \\
\hline Q-2 & 12 & 2 & 2 & 0 & 0 & 0 & 0 & 0 & 0 & 0 & 0 & 0 & 0 & 0 & 2 & 0 & 0 & 0 & 0 & 4 & 2 & 0 & 0 & 0 \\
\hline Q-3 & 14 & 2 & 4 & 0 & 0 & 0 & 0 & 0 & 0 & 0 & 0 & 0 & 0 & 0 & 2 & 0 & 0 & 0 & 0 & 4 & 2 & 0 & 0 & 0 \\
\hline Q-4 & 14 & 4 & 0 & 2 & 0 & 0 & 0 & 0 & 0 & 0 & 0 & 0 & 0 & 0 & 2 & 0 & 0 & 0 & 0 & 4 & 2 & 0 & 0 & 0 \\
\hline Q-1 & 16 & 2 & 6 & 0 & 0 & 0 & 0 & 0 & 0 & 0 & 0 & 0 & 0 & 0 & 2 & 0 & 0 & 0 & 0 & 4 & 2 & 0 & 0 & 0 \\
\hline Q-2 & 16 & 4 & 2 & 2 & 0 & 0 & 0 & 0 & 0 & 0 & 0 & 0 & 0 & 0 & 2 & 0 & 0 & 0 & 0 & 4 & 2 & 0 & 0 & 0 \\
\hline Q-3 & 16 & 2 & 6 & 0 & 0 & 0 & 0 & 0 & 0 & 0 & 0 & 0 & 0 & 0 & 2 & 0 & 0 & 0 & 0 & 4 & 2 & 0 & 0 & 0 \\
\hline Q-4 & 16 & 4 & 2 & 2 & 0 & 0 & 0 & 0 & 0 & 0 & 0 & 0 & 0 & 0 & 2 & 0 & 0 & 0 & 0 & 4 & 2 & 0 & 0 & 0 \\
\hline Q-1 & 20 & 2 & 10 & 0 & 0 & 0 & 0 & 0 & 0 & 0 & 0 & 0 & 0 & 0 & 2 & 0 & 0 & 0 & 0 & 4 & 2 & 0 & 0 & 0 \\
\hline Q-2 & 22 & 2 & 12 & 0 & 0 & 0 & 0 & 0 & 0 & 0 & 0 & 0 & 0 & 0 & 2 & 0 & 0 & 0 & 0 & 4 & 2 & 0 & 0 & 0 \\
\hline Q-3 & 24 & 4 & 10 & 2 & 0 & 0 & 0 & 0 & 0 & 0 & 0 & 0 & 0 & 0 & 2 & 0 & 0 & 0 & 0 & 4 & 2 & 0 & 0 & 0 \\
\hline Q-4 & 24 & 2 & 14 & 0 & 0 & 0 & 0 & 0 & 0 & 0 & 0 & 0 & 0 & 0 & 2 & 0 & 0 & 0 & 0 & 4 & 2 & 0 & 0 & 0 \\
\hline
\end{tabular}


Table - continued from previous page

\begin{tabular}{|c|c|c|c|c|c|c|c|c|c|c|c|c|c|c|c|c|c|c|c|c|c|c|c|c|}
\hline SI-Sybmol & $n^{t}$ & $\stackrel{\oplus}{\mathcal{O}}$ & $\underset{\mathrm{I}}{\mathrm{I}}$ & $\underset{v}{V}$ & $\begin{array}{l}\vee \\
\circlearrowright \\
\wedge\end{array}$ & $\begin{array}{l}\| \\
\bigcup_{N} \\
\underset{I}{I}\end{array}$ & $\underset{T}{\Perp}$ & $\underset{\wedge}{\|}$ & $\begin{array}{l}0 \\
\| \\
\circlearrowright \\
\wedge\end{array}$ & $\begin{array}{l}0 \\
\| \\
\stackrel{⿴ 囗 十}{U}\end{array}$ & $\stackrel{\square}{0}$ & $\mathbb{T}^{N}$ & $\underset{\wedge}{\stackrel{\text { Z }}{\wedge}}$ & 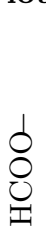 & $\underset{\circlearrowright}{ٍ}$ & 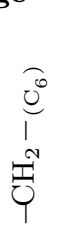 & $\begin{array}{l}\overbrace{}^{0} \\
\vec{v} \\
\vec{U}\end{array}$ & 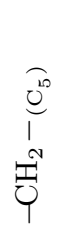 & 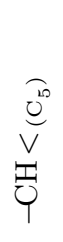 & 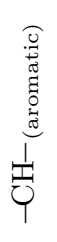 & 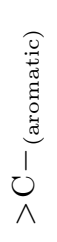 & $\begin{array}{l}\mathscr{I}_{0}^{\infty} \\
0 \\
1\end{array}$ & 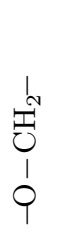 & ф \\
\hline Q-1 & 6 & 0 & 1 & 0 & 0 & 2 & 0 & 2 & 0 & 0 & 0 & 0 & 0 & 0 & 0 & 0 & 0 & 0 & 0 & 0 & 0 & 0 & 1 & 0 \\
\hline Q-2 & 10 & 0 & 2 & 0 & 0 & 0 & 0 & 0 & 0 & 0 & 0 & 2 & 0 & 0 & 0 & 0 & 0 & 0 & 0 & 4 & 2 & 0 & 0 & 0 \\
\hline Q-3 & 5 & 0 & 0 & 0 & 0 & 0 & 4 & 0 & 0 & 0 & 0 & 0 & 0 & 0 & 0 & 0 & 0 & 1 & 0 & 0 & 0 & 0 & 0 & 0 \\
\hline Q-4 & 6 & 0 & 0 & 0 & 0 & 0 & 4 & 0 & 0 & 0 & 0 & 0 & 0 & 0 & 0 & 2 & 0 & 0 & 0 & 0 & 0 & 0 & 0 & 0 \\
\hline Q-1 & 6 & 0 & 0 & 0 & 0 & 0 & 4 & 0 & 0 & 0 & 0 & 0 & 0 & 0 & 0 & 2 & 0 & 0 & 0 & 0 & 0 & 0 & 0 & 0 \\
\hline \multicolumn{25}{|c|}{ mixed functional groups, some groups three times or more } \\
\hline $\mathrm{R}-1$ & 10 & 0 & 5 & 0 & 0 & 0 & 0 & 0 & 0 & 0 & 2 & 0 & 0 & 0 & 0 & 0 & 0 & 0 & 0 & 0 & 0 & 0 & 3 & 0 \\
\hline $\mathrm{R}-2$ & 12 & 0 & 6 & 0 & 0 & 0 & 0 & 0 & 0 & 0 & 2 & 0 & 0 & 0 & 0 & 0 & 0 & 0 & 0 & 0 & 0 & 0 & 4 & 0 \\
\hline $\mathrm{R}-3$ & 14 & 0 & 7 & 0 & 0 & 0 & 0 & 0 & 0 & 0 & 2 & 0 & 0 & 0 & 0 & 0 & 0 & 0 & 0 & 0 & 0 & 0 & 5 & 0 \\
\hline $\mathrm{R}-4$ & 16 & 0 & 8 & 0 & 0 & 0 & 0 & 0 & 0 & 0 & 2 & 0 & 0 & 0 & 0 & 0 & 0 & 0 & 0 & 0 & 0 & 0 & 6 & 0 \\
\hline $\mathrm{R}-5$ & 13 & 4 & 2 & 0 & 0 & 0 & 4 & 2 & 1 & 0 & 0 & 0 & 0 & 0 & 0 & 0 & 0 & 0 & 0 & 0 & 0 & 0 & 0 & 0 \\
\hline
\end{tabular}

